Florida International University FIU Digital Commons

\title{
Exploring Breast Health Perceptions, Behaviors, and Social Cohesion among Ethnically Diverse Black Women
}

Sheila Y.McKinney

Florida International University, smcki004@fiu.edu

DOI: $10.25148 /$ etd.FIDC001967

Follow this and additional works at: https://digitalcommons.fiu.edu/etd

Part of the Community-Based Research Commons, Community Health and Preventive Medicine Commons, Public Health Education and Promotion Commons, Race and Ethnicity Commons, and the Women's Health Commons

\section{Recommended Citation}

McKinney, Sheila Y., "Exploring Breast Health Perceptions, Behaviors, and Social Cohesion among Ethnically Diverse Black Women" (2017). FIU Electronic Theses and Dissertations. 3368.

https://digitalcommons.fiu.edu/etd/3368 


\title{
FLORIDA INTERNATIONAL UNIVERSITY
}

Miami, Florida

EXPLORING BREAST HEALTH PERCEPTIONS, BEHAVIORS, AND SOCIAL COHESION AMONG ETHNICALLY DIVERSE BLACK WOMEN

\author{
A dissertation submitted in partial fulfillment of \\ the requirements for the degree of \\ DOCTOR OF PHILOSOPHY \\ in \\ PUBLIC HEALTH \\ by \\ Sheila Y. McKinney
}

2017 
To: Dean Tomás R. Guilarte

R. Stempel College of Public Health and Social Work

This dissertation, written by Sheila Y. McKinney, and entitled Exploring Breast Health Perceptions, Behaviors and Social Cohesion among Ethnically Diverse Black Women, having been approved in respect to style and intellectual content, is referred to you for judgment.

We have read this dissertation and recommend that it be approved.

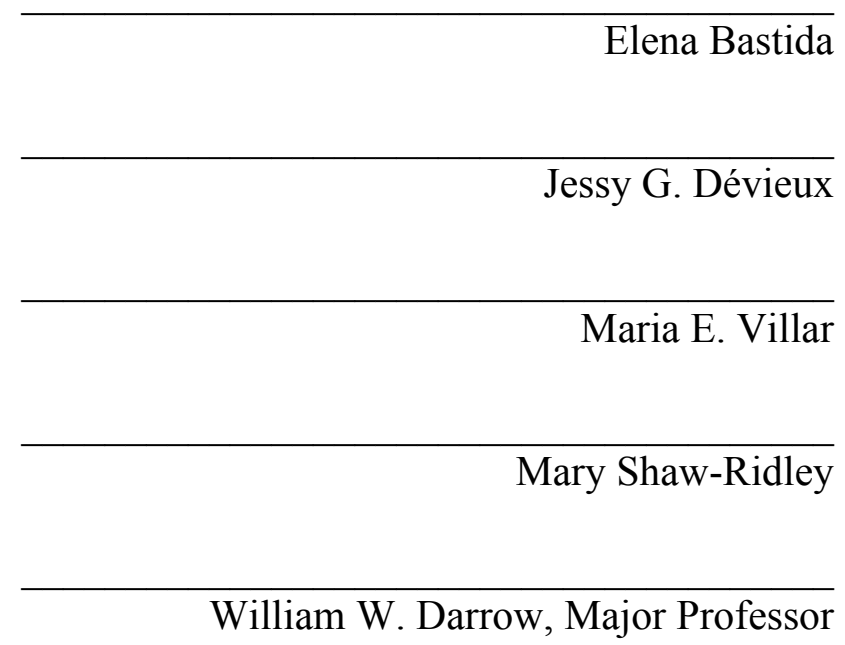

Date of Defense: May 22, 2017

The dissertation of Sheila Y. McKinney is approved.

Dean Tomás R. Guilarte R. Stempel College of Public Health and Social Work

Andrés G. Gil

Vice President for Research and Economic Development and Dean of the University Graduate School

Florida International University, 2017 


\section{DEDICATION}

I dedicate this labor of love to my parents and sisters. They have been my

foundation and inspiration throughout this journey. Salud! 


\section{ACKNOWLEDGMENTS}

I would like to acknowledge the support and guidance of several individuals for without their involvement and commitment to this project this research would not have been possible. To my Major Professor, William Darrow and each of my committee members, Mary Shaw, Jessey Devíeux, Elena Bastida, and Maria Villar, thank you for your leadership and invaluable words of wisdom that you provided throughout this

process. I am forever indebted to each of the members of the Healthy Communities 33311 Community Advisory Board for their calls, outreach efforts, donations, guidance, and commitment kept me standing over the course of two years while engaged in this project. I would also like to acknowledgement the faculty, staff, and students in Robert Stempel College of Public Health and Social Work, staff in the African American Research Library and Cultural Center, members of the National Aid Foundation for Unprovided Children, Inc., and the Westside Gazette for their continued support with this research. Finally, partial funding was provided by the NIGMS Research Initiative for Scientific Enhancement (RISE) Program (NIH/NIGMS R25 GM061347). 


\title{
ABSTRACT OF THE DISSERTATION \\ EXPLORING BREAST HEALTH PERCEPTIONS, BEHAVIORS, AND SOCIAL COHESION AMONG ETHNICALLY DIVERSE BLACK WOMEN
}

by

\author{
Sheila Y. McKinney
}

Florida International University, 2017

Miami, Florida

Professor William W. Darrow, Major Professor

Purpose

Study explored the relationships of ethnic identity, culture, and social cohesion to mammography, cancer screening, and preventive medical visits among African-American and Afro-Caribbean women in Broward County, FL. Purpose was to understand noncompliance to screening recommendations for breast cancer among disadvantaged Black women in an area of high prevalence.

\section{Methods}

A bounded convenience sample of 117 women (49\% African-American and 51\% AfroCaribbean) completed a cross-sectional survey and a subset $(n=87)$ participated in semistructured discussion groups. Both measured perceptions related to breast cancer, defined ethnic identity or culture, and suggested social and cultural factors influence of ethnic identity, culture, and social cohesion on participation with mammograms and preventive 
medical care visits. Survey included the Multi-Group Ethnic Identity Measure (MEIM) and Other-Group Orientation Scale (OGO) for ethnic identity and the Risk Behavior Diagnosis Scale for cancer perceptions. Methods were bivariate, Mann-Whitney U, linear, and logistic regression.

Results

Half of participants (51\%) self-identified as Caribbean. OGO was positively associated with overall attitudes $(p<0.01)$, perceived urgency $(p=0.05)$, and perceived benefit related to breast cancer. Linear regression indicated that Afro-Caribbean women (referent) would perceive less urgency to screen $(\mathrm{p}=0.05)$ and lower risk for breast cancer $(\mathrm{p}=0.03)$ than African-American women. Participants explained that personal and neighborhood cultural norms along with health perceptions along with structural factors connected to access and use of medical services influence Black women's participation in preventive medical services and cancer screening.

\section{Conclusions}

Ethnic identity was associated with women's perceptions of risk, urgency, and benefit for breast cancer screenings. These perceptions may have had a greater influence on the decisions of Afro-Caribbean women not to comply with screening recommendations or participate in preventive medical care actives than for African-American women.

Compliance was also mediated by cultural perceptions of fear, relevance, motivation, and sense of support along with other structural factors. All had contributed to the varying degrees that Black women had sought medical care in this community. Thus, tailoring 
health interventions to account for variations in within-group characteristics is warranted.

[This research was supported in part by NIH/NIGMS R25 GM061347.] 


\section{TABLE OF CONTENTS}

CHAPTER

PAGE

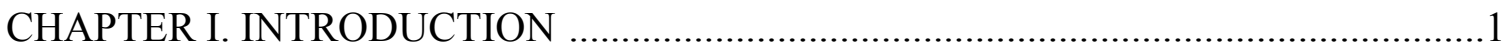

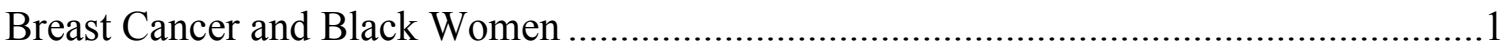

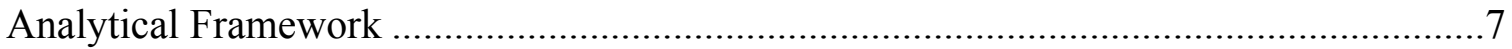

Analytical Framework Components ……………….............................................

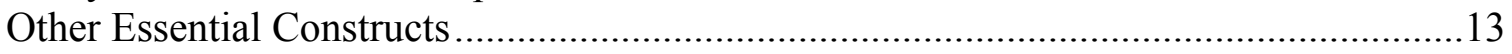

Independent Variable: Importance of Ethnic Identity to Sense of Self .................13

Dependent Variable 1: Health-Seeking Behavior..................................................13

Dependent Variable 2: Attitudes about Breast Cancer ............................................14

Review of Seminal Reports that Shaped the Dissertation ..................................................14

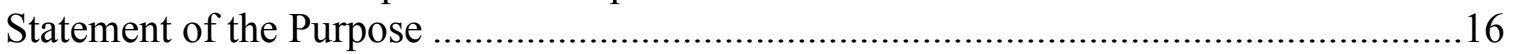

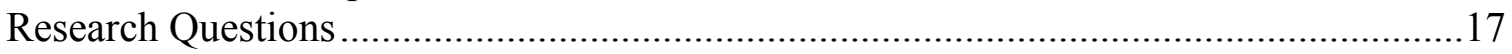

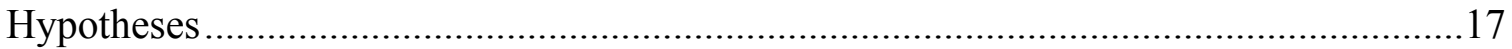

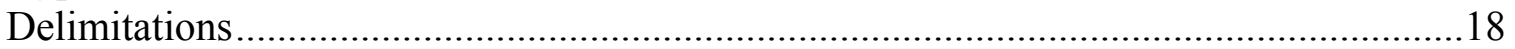

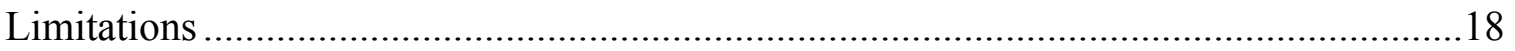

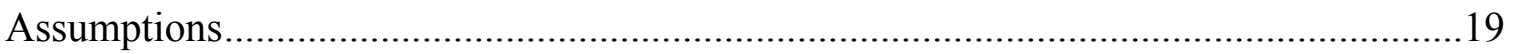

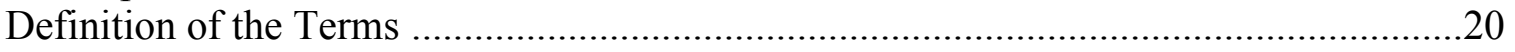

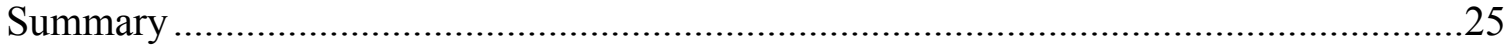

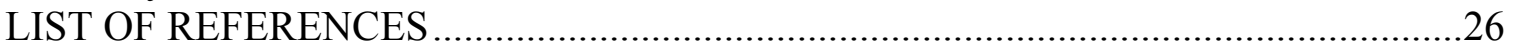

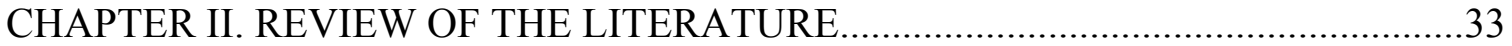

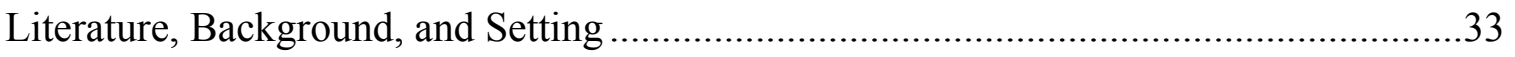

Process to Identify Relevant Literature............................................................................35

The Problem of Breast Cancer Screening in the Community ..............................................36

Breast cancer among Black women nationally and South Florida .........................36

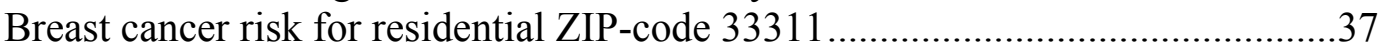

Underlying Factors that Contribute to Breast Cancer Mortality in the Community...........38

Screening adherence and risk for breast cancer. ................................................38

Individual-, structural-, and environmental-level factors. ......................................39

Current cancer screening practice.....................................................................4

Description of Residents and Featured Study Community ...............................................42

Demographic characteristics of women living in ZIP-code $33311 \quad \ldots \ldots \ldots \ldots \ldots \ldots \ldots \ldots . . .42$

Ethnic diversity of Black women in ZIP-code 33311 ...........................................43

Geographical boundaries of target community .................................................44

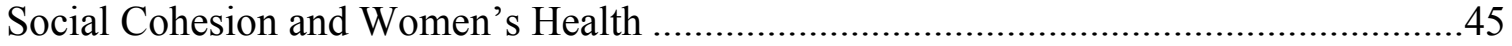

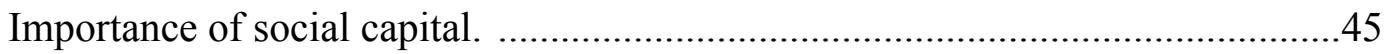

Relationship of social capital and social cohesion to health..................................46

Social capital conceptual framework literature review...........................47

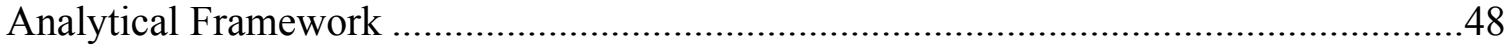

Ethnic identity, connectedness, and Black women ...........................................50

Culture, norms, and Black women................................................................52 


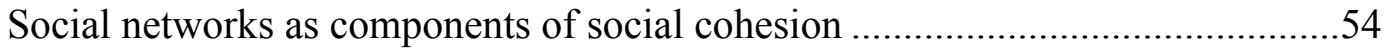

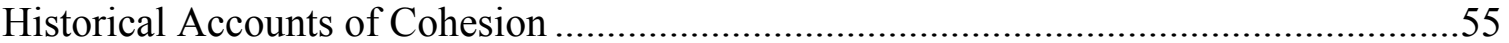

Cohesion among African-American and Afro-Caribbean populations ..................55

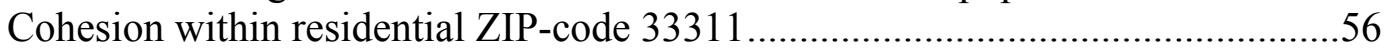

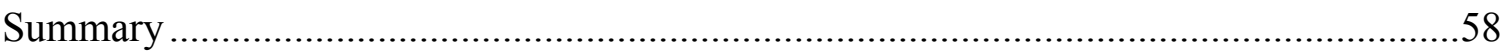

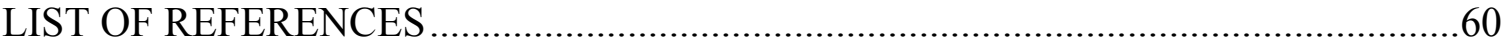

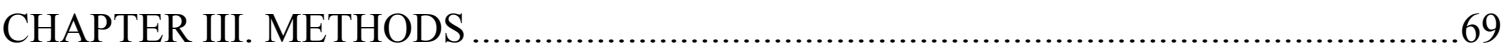

Phase 1: Creation of a Community Advisory Board (CAB) ........................................69

Data Collection During Phase 2 and Phase 3 ................................................................ 71

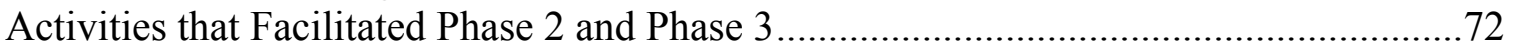

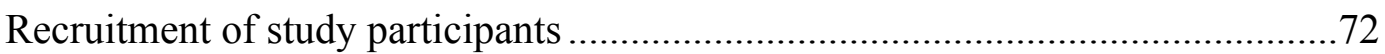

Study sample........................................................ 73

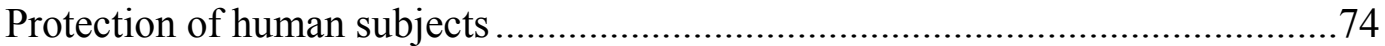

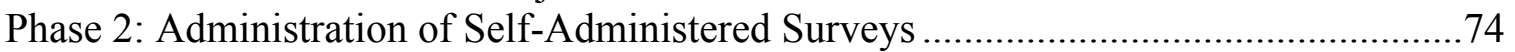

Overview of Survey Content .................................................................................. 75

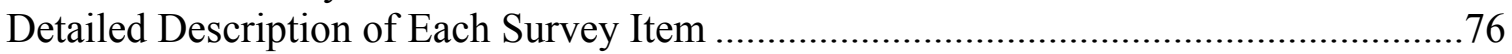

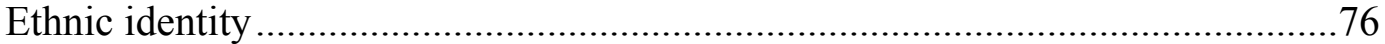

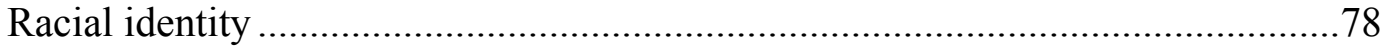

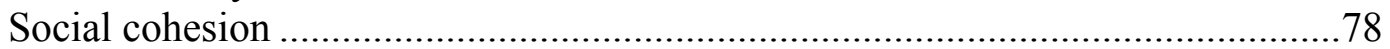

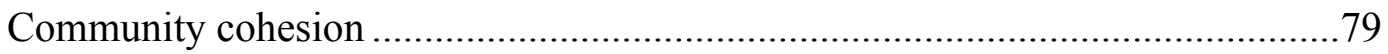

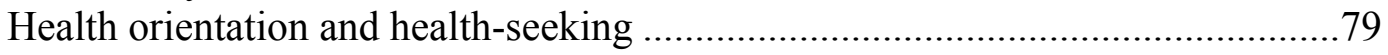

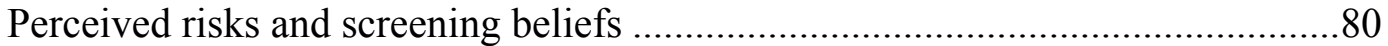

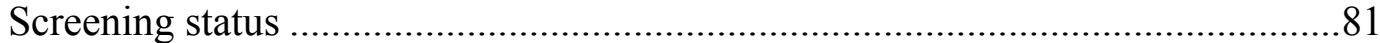

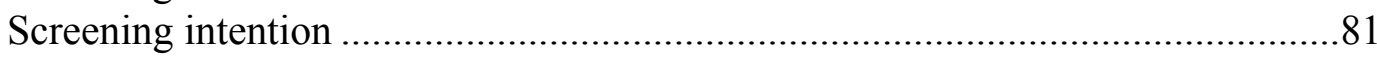

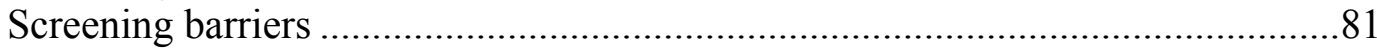

Awareness of Broward Department of Health's screening resources ..................82

Importance of identity to define sense of self ................................................82

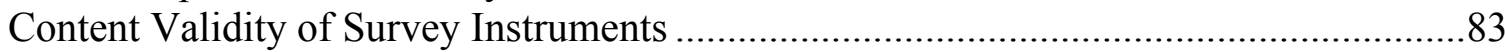

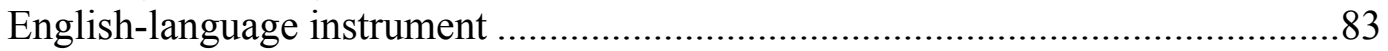

Haitian Creole-language instrument ..........................................................8 83

Phase 3: Participation in Semi-Structured Discussion Groups ....................................84

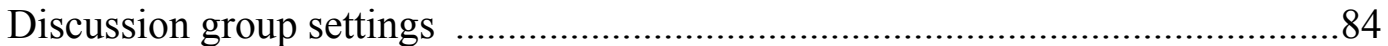

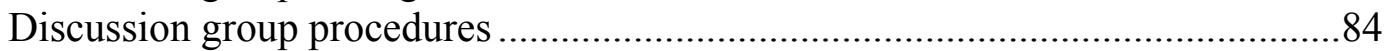

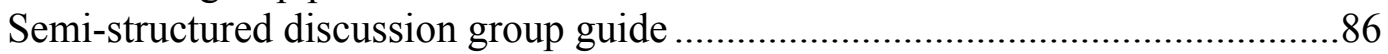

English-language guides............................................ 88

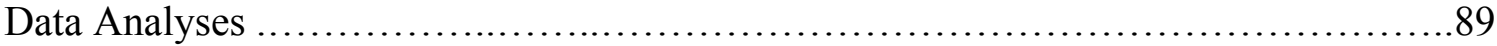

Quantitative Analyses....................................................... 89

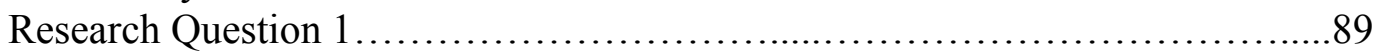

Research Question 2 ................................................... 90

Research Question 3 ..................................................... 90

Qualitative Analyses........................................................ 90

Assembled teams to assist with analyses................................ 90

Steps taken by reviewers in the qualitative analysis.......................91 
Steps taken by researcher to prepare for coding......................... 92

Coding considerations...............................................93

Dissemination Strategies.................................................. 93

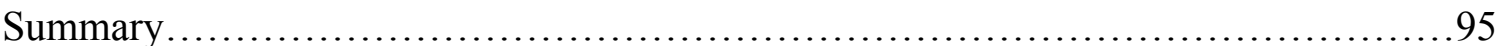

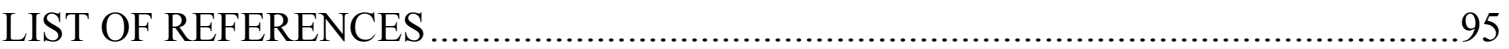

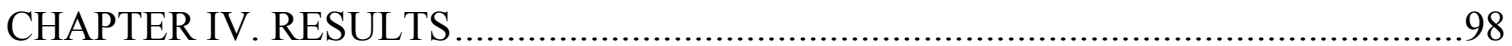

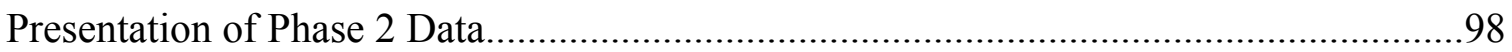

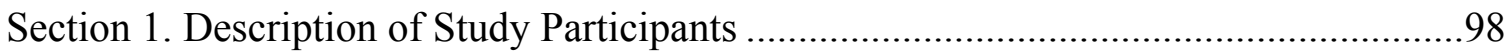

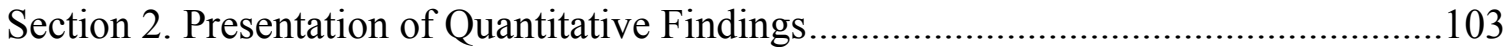

Exploring ethnic differences in health perceptions.........................................103

Ethnic differences in Risk Behavior Diagnosis Scales (RBDS)........................103

Exploring ethnic differences in health-seeking behaviors ................................104

Preventive medical visits. .............................................................105

Medical visits to screen for cancer .................................................106

Multigroup Ethnic Identity Measure (MEIM).....................................106

Influence of ethnic identity on health-seeking behaviors ................................109

Likelihood to see a doctor as a preventive medical visits......................109

Likelihood to ever see a doctor to screen for cancer .............................110

Relationship of Ethnic Identity and Social Cohesion among Black Women ......111

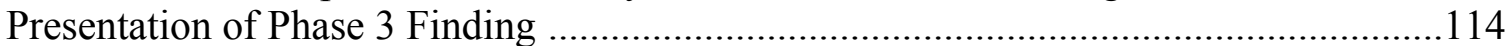

Section1. Ethnic Composition of the Semi-Structured Discussion Groups.....................114

Section 2. Qualitative Findings from Phase 3 ......................................................115

Women's Perceptions about Mammography and Breast Cancer ...............................115

Expectation of Pain .................................................... 115

Women Characterize Culture..................................................... 118

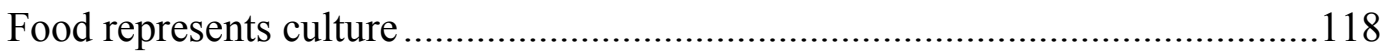

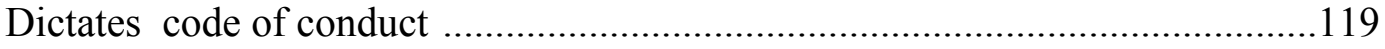

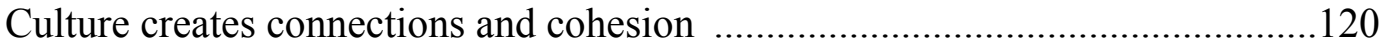

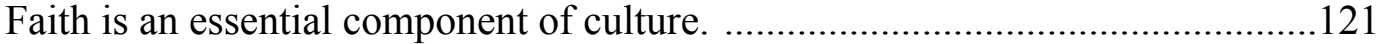

Greater trust in holistic options over medical approaches....................122

Influence of Culture and Community Norms on Health-Seeking Behaviors ..................123

Culture and Community Norms of African American Women..........................123

Adoption of holistic practices as community tradition................. 123

Leaders influence health behaviors....................................................124

Culture and Community Norms of Haitian Women ...........................................125

Faith is an essential characteristic of Haitian culture ................. 125

Class, income and immigration status intervene with health-seeking behaviors.................................................... 127

Preventive care differentiates health care systems....................129

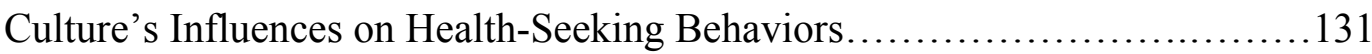

Women have conflicting views on necessity of screening ..............131 
Women understand importance of prevention yet refrain from care .....132

Fear can mediate behavior........................................ 132

Motivation can mediate behavior.................................135

Pride can mediate behaviors $\quad$.....................................136

Support mediates behavior....................................137

Structural Impediments...................................................... 141

Burdensome process to qualify for economic support ......................141

Limited accessibility................................................... 143

Community's Recommendations to Improve Women's Participation ...............144

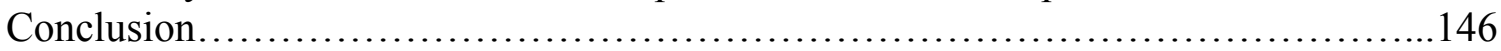

Summary of Major Quantitative Findings from Phase 2 ...........................146

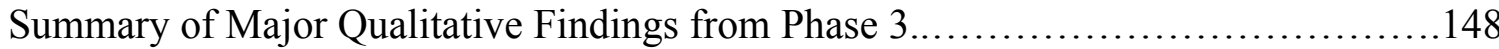

CHAPTER V: DISCUSSION ................................................ 151

Summary of the Study ........................................................ 151

Statement of the Problem........................................................ 152

Purpose of the Study....................................................... 153

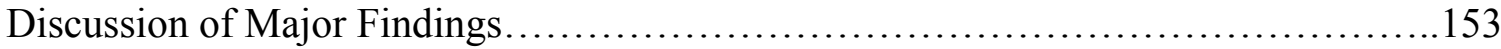

Ethnic Variations in Health Perceptions..................................154

Health Perceptions and Health-Seeking Behaviors .............................. 155

Relationship of the MEIM to Women's Health Perceptions ...................156

Explanation for Behaviors of Late-Diagnoses and Non-Adherence..............158

Recommendations....................................................... 167

Recommendations for Practice....................................... 167

Recommendations for Improving Research.............................171

Recommendations for Future Research...................................172

LIST OF REFERENCES .............................................. 173

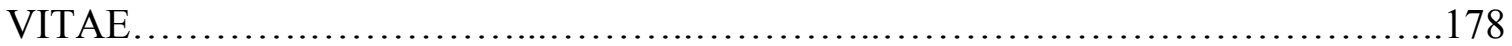




\section{LIST OF FIGURES}

FIGURE

PAGE

Figure 1. Structured Characteristics, Social Cohesion, and Social Capital: Carpiano's

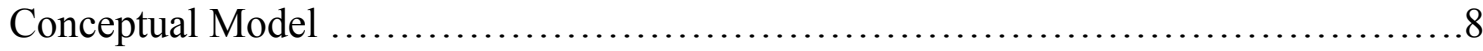

Figure 2. Culture, Ethnic Identity, Social Cohesion, Neighborhood Social Capital, and Breast Cancer Screening: A Reconceptualization................................ 9

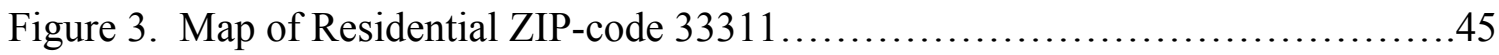

Figure 4. Structured Characteristics, Social Cohesion, and Social Capital: Carpiano's

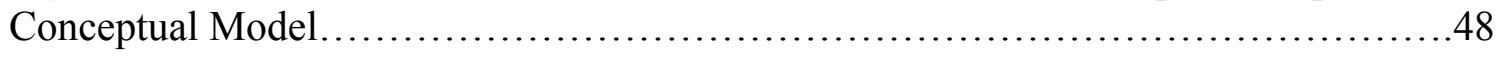

Figure 5. Culture, Ethnic Identity, Social Cohesion, Neighborhood Social Capital, and Breast Cancer Screening: A Reconceptualization...................................49

Figure 6. Order of Research Study Phases 1-3: Community Advisory Board, Administration of Self-Administered Survey, and Participation in Semi-Structured

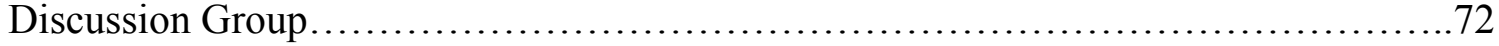

Figure 7: Percentage of Women with Select Health-Seeking Characteristics and

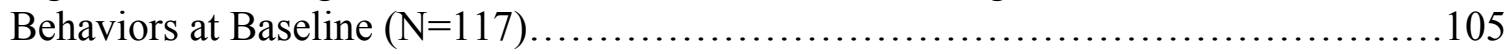

Exhibit 8: Future Actions to Engage Public Health Personnel in the Community........170 


\section{CHAPTER I. INTRODUCTION}

The purpose of this exploratory study was to develop an understanding as to why an ethnically diverse group of Black women who self-describe as African American and Caribbean and live within a bounded Broward County community are not complying with screening recommendations for breast cancer. Three aims derived from this purpose were to: (a) explore perceptions and health-seeking behaviors about breast self-exams and mammograms among a community of ethnically diverse Black women, (b) determine if ethnic differences in their perceptions and health-seeking behaviors exist, and (c) characterize cultural and ethnic identity as a tool to address their health-seeking behaviors.

\section{Breast Cancer and Black Women}

Breast cancer mortality rates among Black women remain an urgent public health issue. Black women continue to be diagnosed at later stages, have the greatest likelihood of developing the most aggressive forms of breast cancer, and have the lowest survival rates among all ethnicities (CDC, 2015; ACS, 2015). Yet, nationally incidence rates over the past five years have been decreasing among white women while, during the same period, the rates for Black women continue to increase (ACS, 2015). Moreover, Black women continue to have the highest mortality rates among all racial and ethnic groups, with Black women living in the Southern region of the US now having the highest rates in the nation (DeSantis, 2015).

Health disparities related to breast cancer create a concern for the South because Black women comprise over half of the Black population, with an estimated $53 \%$ of these women falling within the age group that should be receiving ongoing breast cancer exams 
(US Census, 2015). The failure of Black women to adopt and maintain recommended breast screening behaviors has been associated with the disproportionately higher rates of mortality found in Black women (Akinyemiju, et al., 2013). The problem becomes more complicated in that along with the rising mortality rates in southern states is a concurrent increase in the number of Black women diagnosed with this disease in its later stages (CDC, 2015; ACS, 2015). Both factors have significantly contributed to Black women having the lowest survival rates for breast cancer among all ethnicities (CDC, 2015; ACS, 2015). Added to these issues of access and use of screening resources are the complexities presented by the influence of racism, poverty, culture, and faith on their health behaviors (Musgove, Allen \& Allen, 2002; Brondolo, Gallo \& Myers, 2009; Danfort, 2013; Pater et al., 2014).

Interestingly, over 15 years of evidence has been collected on breast cancer risk, prevention, mortality, adherence, health behaviors, and health interventions that have targeted African-American women (Padamsee, Wills, Yee \& Paskett, 2017; ACS, 2016; DeSantis et al., 2016; ACS, 2015; Danforth, 2103; Lipkus, Iden, Terrenoire \& Feaganes, 1999; Erwin, Spatz, Stotts, Hollenberg, 1999). In this extensive body of work the inclusion of non-American Black women is rarely specified and explicit study of these issues with non-American Black women is underexplored (Brown et al., 2017). To the extent that this knowledge can be appropriately applied to non-American Black women remains unclear.

This dissertation explored the problems of late diagnoses and mortality among ethnically diverse Black women within or near to one residential ZIP-code of Broward County, FL to gain a better understanding of the breadth of this problem and to offer 
community-driven solutions. Research has clearly shown that Black women carry the greatest health burden from breast cancer, but this evidence has not substantiated whether the burden is distributed evenly across the various populations that represent Black women in America (DeSantis, 2015). The ethnic diversity of ZIP-code 33311, where $85 \%$ of the population self-describes as Black, yet, almost $24 \%$ are Caribbean and foreign born, served as an ideal, natural setting to develop an understanding of the local context for why late diagnoses with breast cancer and related mortality remain critical issues among Black women.

Research suggests that ethnic identity, immigration status, and culture can increase the difficulty of ethnically diverse Black women to access and use health services (Sussner, 2010; Consedine, 2012). As in the Sussner study, the Black women who live in 33311 primarily represent two groups: a) either African American with families that have ties to the community for generations or b) women that have newly immigrated to the community with strong ties to their Caribbean cultures. Therefore, this dissertation sought to establish whether similar patterns exist between Afro-Caribbean and African-American women in their perceptions about cancer and mammography, as well as their participation in preventive medical services which to date, has not been explored in Broward County.

Ethnic identity and culture are mechanisms that can be used to create a socially supportive environment in a community that promotes women's health (Airhihenbuwa, Kumanyika, Tenhave \& Morissink, 2000). A key element within communities is the active engagement of the social networks that promote the subjective and objective welfare of members of the community (Szreter \& Woolcock, 2004). Often these 
networks are formed by a connectedness among residents along common traits that include shared beliefs, values, and norms dictated by their culture. To the extent that networks find similarity, support, and mutual interest, so too will the likelihood of cohesive relationships within and across networks exist (Kim \& Kawachi, 2011). In terms of a community where almost $30 \%$ have a common experience of being born outside of the US, the natural tendency will be for women to gravitate and connect with women having similar histories, cultures, experiences, or ethnic identities (Ray, 2003). Evidence of connecting and support along common values and beliefs suggests the willingness and capacity of members of the network to work together and speaks to the overall social cohesiveness that exists in the communities (Kawachi, Kennedy, \& Glass, 1999; Putnam, 2000). These networks can serve as avenues of support for women to access screening services and act as effective points of entry into the community to promote cancer screening. The willingness of separate African-American and Afro-Caribbean groups to collectively mobilize along issues that promote the betterment of all Black women living in 33311 is unknown.

Researchers have begun to appreciate that culture, ethnicity, and interpersonal connections that lead to cohesion may differ among Black people who live in the US (Tsai et al., 2017; Molina \& James, 2016). In turn, these differences often create variations in essential behavioral factors that influence risk perceptions, mortality, and action that promote disease prevention. For example, research has revealed that breast cancer survival among Black women is associated with their ethnicity (Caribbean versus African American) (Camacho-Rivera, Shapira \& Taioli, 2014). These studies further demonstrate that ethnicity in conjunction race are powerful tools to address breast cancer 
screening among Black women. However, this work is concentrated in a limited region of the US, primarily, the northeast; the scope of this research is broad and lacks replication. Expanding this research in South Florida given the ethnic diversity the Black population seems appropriate.

Finally, inability to pay for medical services remains a leading barrier for many Black women to access and use mammography (Plescia, Wong, Pieters \& Joseph, 2014). A recent evaluation of the impact of health reform on the National Breast and Cervical Cancer Early Detection Program (BCCEDP) reports that the national program will still need to support approximately 1.7 million poor and underserved women to access mammograms. These women will not receive any funding from the combined pooling of money across the Affordable Care Act, expansion of Medicaid, and full participation by all states in the health insurance marketplace (Plescia, Wong, Pieters \& Joseph, 2014; Levy, Bruen \& Ku, 2012).

Similar to women who live in ZIP-code 33311 a higher proportion of women needing financial assistance will be less educated, Black, and ethnically diverse. Public health will find it harder to reach and serve these women because differences in language, limited literacy, nativity, and culture can keep them disconnected from the broader community and increase their isolation from public health services (Plescia,Wong, Pieters \& Joseph, 2014). This then becomes an opportunity to test the existing evidence that suggests the relationships between culture, ethnicity, and social cohesion can be used as tools to better engage Black women in health care services (Carpiano, 2007). Most notably, evidence will improve the efficacy of services and increase the proportion of 
African-American and Afro-Caribbean women who become engaged with preventive care services and see a doctor to complete a mammogram in Broward County.

Factors related to culture, ethnic identity, and social cohesion offer competing explanations for why women in this community tend not to engage in preventive medical exams nor complete mammograms. Prevailing assumption in the evaluation produced by Plescia and colleagues (2014) is that the provision of health insurance to cover the cost of a mammogram for lower income women will increase the proportion of women who become and remain compliant with the breast cancer screening recommendations. In Broward, however, $76 \%$ of the women who live in ZIP-code 33311 receive some type of health insurance coverage (TownChart.com, 2016; FLDOH, 2016). Yet $48 \%$ of the women who completed mammograms that lived in ZIP-code 33311 were diagnosed with breast cancer in its later stages (Kobetz \& Parker, 2010). The fact that so many of these same women have refrained or delayed obtaining breast exams suggests that noncompliance to screening recommendations is happening for reasons other than affordability in this community.

Other possible reasons could be women's perceptions of fear and pain related to the exam or a low perception of risk for developing breast cancer that contribute to their delay or avoidance with the mammogram (O’Neal eta 1., 2014; Jones et al., 2014). Some women may have adopted cultural practices or internalized cultural beliefs that discredit the necessity of preventive medical exams or mammograms to promote better health (Peek, Sayad \& Markwardt, 2008). Then others may lack the necessary support among family, friends, and other community networks that prioritize healthier behaviors (Molina et al., 2015; Klassen \& Washington, 20008). Therefore, establishing a general 
understanding of the women's various cultural norms, perceptions about health, and behaviors to engage with the health care system will increase the effectiveness of cancer prevention interventions in Broward.

\section{Analytical Framework}

Given that so many questions remain unanswered regarding the lack of adherence to screening recommendations among Black women in Broward, and in the 33311 community specifically, this dissertation has sought to add clarity to why Black women have had difficulty accessing and using preventive medical services including mammograms. Screening compliance is often a complicated process, particularly for women who live in communities where many residents are characterized as having poorer health status, are disenfranchised, and many are also foreign born (Urban League of Broward County, 2010; US Census, 2016).

The conceptual framework selected for this study was adapted from Carpiano's (2007) model of social capital (Figure 1). His model illustrates various pathways that lead to health outcomes within modern, urban communities. The major components found among these pathways include individual-level traits among the residents, social cohesion within a community, the values and connections that create cohesion in the communities, and the presence of social capital in the community. Carpiano's model also accounts for the influence of external, structural-level factors that act upon a community's ability to promote or seek healthier alternatives. 


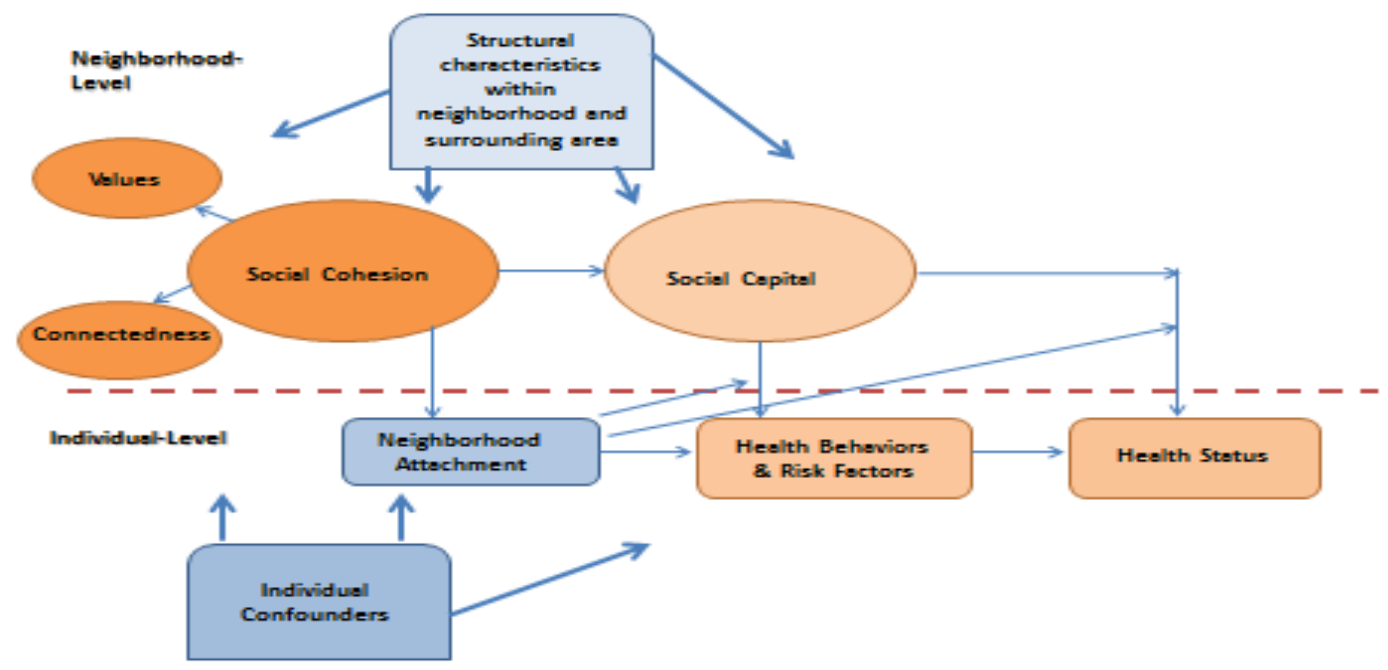

Figure 1. Structured Characteristics, Social Cohesion, and Social Capital: Carpiano's Conceptual Model

The scope of this dissertation's research was bound by practical limitations (i.e., limited funding and staff) and, as a result, the exploration of Carpiano's model was limited to three constructs. Specifically, the relationships found among social cohesion, values, and connectedness, and their influence on the health status of residents within this community. Based upon the cultural, community, and social dynamics that characterize African-American and Afro-Caribbean people, modifications were made to the constructs that represented values and connectedness. The conceptual model illustrates that the term values and connectedness were relate to social cohesion. The construct cultural norms best representss the values of women of African descent and their communities and that ethnic identity more accurately acts as the cornerstone of connectedness for ethnically diverse Black women. For this dissertation it has been assumed that ethnic identity and culture contribute to each other and are not mutually exclusive concepts, therefore the 
conceptual framework for this dissertation illustrates a bidirectional relationship between ethnic identity and culture (Figure 2).

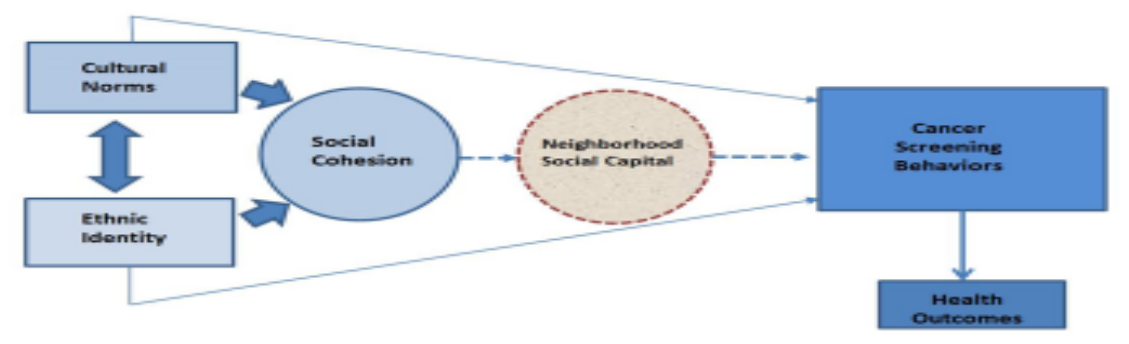

Figure 2. Culture, Ethnic Identity, Social Cohesion, Neighborhood Social Capital, and Breast Cancer Screening: A Reconceptualization

\section{Analytical Framework Components}

Figure 2 illustrates the components of the analytical framework used for this dissertation. The next section will provide further detail of the three primary components of this model (social cohesion, ethnic identity, and culture) and describe their application to this study. Following this description will be an overview of the other primary research concepts used in this research that served as dependent variables.

Social cohesion. Social cohesion is defined as patterns of social interaction and values (such as network formation and ties, familiarity, and mutual trust) that lead to social capital. They serve as intermediaries between structural antecedents and social capital, and are necessary foundations for establishing social capital within neighborhoods (Carpiano, 2006). These interactions become the mechanism that facilitates individuals to come together and promote healthier conditions that provide 
direct benefit to self and members of the network (Szreter \& Woolcock, 2004). This study, like Carpiano's, views social cohesion as a separate and underlying precursor to social capital. The essential difference between the two is that cohesion leads to social capital and attends to the relationships, particularly the trust and reciprocity that exists among members of a network that can facilitate mutual benefit for individual members. Social capital attends to the collective benefit afforded by networks as a resource to advocate for the "community," to acquire resources that benefit the health of the community, or lead to societal benefit. In this study, social cohesion is used to: (1) comment on the nature of common ties, mutual trust, values, and familiarity between African American and Afro-Caribbean women in the 33311 ZIP--code, and (2) suggest areas to motivate members of the networks to support breast screening. So, social cohesion is necessary to create social capital. Without cohesion, a community would have no social capital to draw upon.

Social cohesion is operationalized by three items within the cross-sectional survey: (1) what is your general feeling of trust that you have for your neighbors, (2) would you say that most of the time people in your neighborhood try to be helpful, and (3) have you volunteered or been a member of any of these organizations in this community (Kiwachi, 1999).

Ethnic identity. Ethnic identity represents two areas of identity as described by Phinney (1999) as both the self-described label taken on by members of the nondominant ethnic groups in the US and the psychological implications of that membership. Ethnicity is used to refer to broad groupings of Americans on the basis of both race and 
culture of origin that are used by the US Census (African American, Asian, American Indian/Alaska Native, Native Hawaiian/Pacific Islander, Hispanic).

The theoretical origin for ethnic identity comes from psychology and its importance to the field represents three areas. These include: (a) the cultural values, attitudes, and behaviors that distinguish ethnic groups; (b) the subjective sense of ethnic group membership (i.e., ethnic identity) that is held by group members; and (c) the experiences associated with minority status, including powerlessness, discrimination, and prejudice (Phinney, 1996).

For this study, ethnic identity represents one of the key independent variables that may predict social cohesion (another independent variable) among African-American and Afro-Caribbean women in the community. The assumption is that women will develop a bond with other women dictated by the level of familiarity that is created by the women having a shared ethnic history or origin. Their self-described membership is also used for analytical purposes to compare outcome variables across the women (i.e., African American versus Caribbean). The psychological context of identity will suggest the level of importance placed by African-American and Afro-Caribbean women on being a member of their respective ethnic group and suggest whether this importance influences their health screening behaviors. Findings could be used to tailor future screening interventions.

Ethnic identity was operationalized in two areas of this dissertation. Both are within the cross-sectional survey. First, women were asked to define their ethnic group by selecting up to two ethnicities from among a list of 14 known ethnic groups relevant to women of African descent that were known to live in 33311 at the time of 2010 US 
Census. This list included residents that self-described as Black-African American, as well as, other Blacks that also described as Caribbean, West Indian, European, Canadian, or of origin from one of the US Caribbean islands and other locations. The crosssectional survey also included the 12-item scale of the Multi-Ethnic Identity Measure and the Other Group Orientation scale to capture the psychological components of identity (Phinney, 1992).

Culture. Drawing upon a cross-disciplinary context of cultural and community psychology, culture is defined as "a focus on the implicit and explicit patterns of meaning, practices, and artifacts distributed throughout the contexts in which people participate, and on how people are engaged" (as cited in O'Donnell \& Tharp, 2011, p. 23). Culture can be expressed by residents of a community in their "language, speech patterns, artifacts, music, values, and behavioral norms" (p.23).

Based upon this definition culture can provide a contextual reference for women that guides the motivation, rationalization, and prioritization to seek opportunities for a mammogram and complete the exam. The inclusion of culture as a prominent independent variable is based upon the research of Sussner and colleagues (2010). They studied the influence of cultural variations among ethnically diverse Black women regarding their perceived benefits and barriers related to genetic testing for breast cancer. They chose to operationalize cultural identity by using a measure of Africentrism (Grills, 1996) which was not found to be statistically significant in their study (Sussner et al., 2010). However, this could have been a function of the selected measure and the context of these items would only have relevance to individuals raised with an Americanized experience. Therefore this dissertation drew upon Sussner's study and operationalized 
cultural identity using qualitative methods where community members provided their perspectives and context for culture and its relationship to health behaviors.

\section{Other Essential Constructs}

One independent and two dependent variables comprise the remaining essential constructs of this research. The following section provides the rationale for these variables and describes how each was operationalized for this study.

\section{Independent Variable: Importance of Ethnic Identity to Sense of Self}

An underlying assumption of this study is that ethnic identity varies in its influence on decision-making and behaviors based upon the level of perceived importance that ethnicity holds within the psyche of the woman. Therefore, greater internal importance will heighten the awareness of the ethnicity of others, and may sway behaviors based upon the alignment to the priorities set by the ethnic group. Similar questions were included in the 2005 Citizenship Survey administered in London to study social cohesion nationally. Three of the items included in the Citizenship Survey were used to explore the internalized importance of ethnic identity (Laurence, 2011). This measure was operationalized by summing across the three questions to create one indicator of importance.

\section{Dependent Variable 1: Health Seeking Behavior}

Health-seeking behavior is represented by two distinct actions in the care setting. The first is likelihood of seeing a doctor before being sick. The second behavior is likelihood to see a doctor to screen for cancer. The first behavior represents the likelihood that a woman living in the target community would engage in a preventive medical visit. It was selected because the decision to see a doctor is a complex process 
whereby barriers such fear, avoidance, and fatalism can influence the decisions made by Black women (Peek, Savad \& Markwardt, 2008; Giersch et al., 2009; Allen, Mars, Tom \& et. al., 2013; Jones, 2014). Their influence on behavior may vary based upon the potential severity of the outcome (Poonawalla, Goyal, Mehrotra, Allicock, \& Balasubramanian, 2014). So the general preventive care visit was perceived as routine and less threatening while the second behavior screening for cancer was more threatening. Therefore, the two behaviors were included as items in the cross-sectional survey and represent the overall dependent variable of health-seeking behavior.

\section{Dependent Variable 2: Attitudes about Breast Cancer}

Baseline information on the general sensibilities of Black women in ZIP-code 33311 about breast cancer and screening does not exist and this information would be invaluable to inform the creation of health interventions that increase screening activity. The questions that comprised the attitude measures were derived from the Risk Behavior Diagnostic Scale (RBDS) (Witte et al., 1996). All 12 items that represent four distinct beliefs were included in this study. Summing all 12 questions became the measure of overall attitude towards breast cancer. The four subscales measure perceptions of disease severity, risk, self-efficacy, and response efficacy. Summative variables were also created to represent each sub-scale from the original 12 items included in the crosssectional survey.

\section{Review of Seminal Reports that Shaped the Dissertation}

Four seminal works influenced the conception of this study. Collectively, they are the foundation of this study and shaped the rationale for conducting this study. Each offered a unique view of the context of the issues presented in the introduction. 
The first report, "Closing the Gap, the State of Black Broward: Health Report" (2010) was created as a call to action by Sunshine Health and the Urban League of Broward County to prioritize attention to a failing health system for Broward's Black residents. Their call was to engage citizens and stakeholders to make the necessary changes that would promote equity for non-Hispanic Blacks that disproportionately present with an expansive list of health problems This report highlighted that the issues of individuals having poor health were complex, and stemmed from multiple, multi-layered factors originating from the individuals, the environment, community infrastructure, and governance. More importantly, voices of residents and community stakeholders needed to be actively engaged. This report influenced the selection of Carpiano's (2007) framework to guide this research and the inclusion of key informant interviews and semi-structured discussion groups with women from the community as data collection methods.

The second report, "Cancer Data for South Florida" (Kobetz \& Parker, 2010) has served since 2010 as the only analysis of breast, cervical, colon, and prostate cancers by geographical ZIP-codes in South Florida. The fact that for the past six years no other resource has been created and made available to public health advocates as guidance and information highlights the systemic factors that detract from the ability of public health and other stakeholders to improve local health conditions. Drawing on the work of the University of Miami, this report informed the selection of: (1) breast cancer as the target disease for study and (2) 33311 as the research site to conduct this research. The use of community residents as key informants to provide current perspectives on the issues that they face to screen for breast cancer was adopted to compensate for the absence of more current data specific to ZIP-code 33311. 
Sussner and colleagues (2010), provided evidence that reliance on a monolithic view of Blacks in the US regarding their health-related perceptions, risk and attitudes may be responsible for Type 1 error in public health research. Their findings support a growing body of evidence that factors such as nativity, country of origin, acculturation, culture, and ethnicity can influence health outcomes among Blacks. Their study led to interest of replicating their measures of ethnic identity which used the multi-ethnic identity measure (MEIM) to explore common outcomes among a similarly diverse population.

Finally, Carpiano (2007) provided an ecological framework for social capital that was appropriate for the complexities of the health disparities described in the other seminal reports. However, in Broward County, as in many other communities nationally, public health information does not adequately reflect the rich ethnic diversity of the community. Therefore, this study focused on a limited component of his model (social cohesion, values, and connectedness) as a viable option to build the capacity of researchers to scale their explorations to the fuller model. Collectively, the four reports became the foundation of this dissertation. They also set the stage for what became the purpose, hypotheses and questions addressed in this study. These components are presented in the next section.

\section{Statement of the Purpose}

The purpose of this exploratory study was to develop an understanding as to why an ethnically diverse group of Black women who self-describe as African American and Caribbean and live within a bounded Broward County community are not complying with screening recommendations for breast cancer. Three aims derived from this purpose 
were to: (a) explore perceptions and health-seeking behaviors about breast self-exams and mammograms among a community of ethnically diverse Black women, (b) determine if ethnic differences in their perceptions and health-seeking behaviors exist, and (c) characterize cultural and ethnic identity as a tool to address their health-seeking behaviors.

\section{Research Questions}

Major research questions were:

\section{Quantitative}

1. What are the perceptions and health-seeking behaviors about cancer among ethnically diverse Black women (African American and Afro Caribbean)?

2. What is the relationship between ethnic identity and health-seeking behaviors among African-American and Afro-Caribbean women?

3. What is the relationship between ethnic identity and social cohesion among AfricanAmerican and Afro-Caribbean women?

\section{Qualitative}

4. Are there differences in perceptions about cancer and mammograms between AfricanAmerican and Afro-Caribbean women?

5. How do ethnically diverse Black women characterize ethnic identity and culture?

6. How does ethnic identity and culture influence the health-seeking behaviors of ethnically diverse Black women?

\section{Hypotheses}

Null-hypotheses were: 
1. $\mathrm{H}_{0}$ : African-American and Afro-Caribbean women do not have a statistically significant difference in their perceptions and health seeking behaviors about cancer.

2. $\mathrm{H}_{0}$ : Ethnic identity will not have a statistically significant influence on the healthseeking behaviors of African-American and Afro-Caribbean women.

3. $\mathrm{H}_{0}$ : Ethnic identity will not have a statistically significant influence on the detection of social cohesion among African-American and Afro-Caribbean women.

\section{Delimitations}

The study was delimitated by the following:

1. Black women 40-85 years of age, living in or adjacent to the target ZIP-code 33311.

2. Women who self-described as Black or Black/mixed race.

3. Women who self-described as African American or Caribbean/West Indian.

4. Women who spoke English or Haitian-Creole.

5. Women able to complete the cross-sectional survey by reading the document in English, Haitian-Creole, or with the assistance of audio or verbal support in English or verbal support in Haitian-Creole.

6. Women who knew that they were born in the US or outside of the US.

\section{Limitations}

The study was limited by the following:

1. It is a cross-sectional study and no causality could be implied.

2. A convenience sample was used to enroll women into the study, limiting the generalizability of these findings to the perspectives and behaviors of the broader community. 
3. The majority of the women in this study reported a lower income and educational attainment compared to other Black women in Broward; consequently, their responses may not be generalizable to women with higher incomes and levels of education.

4. The majority of the Caribbean women and their parents were born outside of the US, therefore, their perceptions and behaviors may not represent other Caribbean women who were born in the US or have a parent who was born in the US.

5. Most of the African-American women have lived the majority of their lives in Florida; hence, their perceptions and behavior responses may not represent those women who have established residency in neighborhoods outside of Florida.

6. Data were self-reported and were not verified with other sources of information.

\section{Assumptions}

The following assumptions guided this study:

1. Women were honest in their responses to the survey and discussion questions.

2. Women understood the questions asked of them.

3. The selected data collection sites were easily accessed by the women.

4. Women were comfortable with the facilitators (both English and Creole speaking).

5. Placement of posters and other recruitment materials in the African-American

Research Library, churches, and other local businesses were able to recruit eligible women for the study.

6. Participating in on-air radio interviews at local Creole-language radio stations would recruit eligible Haitian women for the study.

6. The focus group and survey could be completed within a 2-hour window. 


\section{Definition of the Terms}

Black or Black Women - According to the U.S. Office of Management and

Budget, "Black or African American" refers to a person having origins in any of the Black racial groups of Africa. The 2010 Census includes persons who checked the "Black" checkbox. Selecting this checkbox may also include individuals who reported

- African American

- Sub-Saharan African (e.g., Kenyan, Nigerian)

- Afro-Caribbean (e.g., Haitian, Jamaican) (Jones \& Johnson, 2010).

Civic Engagement - Working to make a difference in the civic life of our communities and developing the combination of knowledge, skills, values and motivation to make that difference. It means promoting the quality of life in a community, through both political and non-political processes (Ehrlich, 2000).

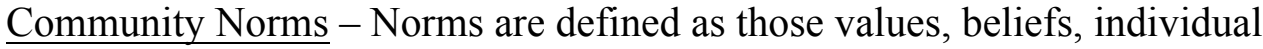
characteristics, and behaviors shared by most people in a "group," here represented as the broader community and the collective by ethnicity. In other words, norms are what most people value, believe, and do. The group could be a physical community (like a town or county), students within a school, employees of an organization or workplace, people linked by a common experience (such as first-time parents), or any other affiliation that allows individuals to establish a group identity. Norms can be positive and negative. (Linkenbach \& Otto, 2014)

Comprehensive Breast Health Strategy - Women incorporating breast self-exams, clinical exams, and mammograms in their ongoing activities to take care of their breast and facilitate early diagnosis of possible cancer in the breast. The American Cancer 
Society (ACS) (2016) promotes mammograms for women, but says that evidence does not support use of either the clinical breast or self-breast exam. ACS advises that "all women should be familiar with how their breasts normally look and feel and report any changes to a health care provider right away" (ACS, clinical breast exam and self-breast exam section, para. 1).

Cultural Identity - Defined as a "collective sense of consciousness that can audibly or silently reveal itself through history and language" (Airhihenbuwa \& Liburd, 2006, p. 491). Effects of this identity may or may not be internalized the same way as an individual or as a group, or across members that make up the group; nor does culture remain static over time. Airhihenbuwa \& Liburd (2006) explained that culture is reinforced both positively and negatively, visible and not, creating the social context experienced by people.

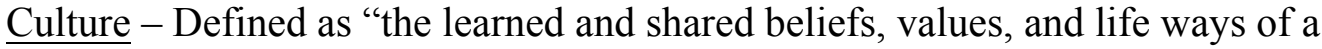
designated or particular group which are generally transmitted intergenerationally and influence one's thinking and action modes" (as cited in Egede, 2006, p. 668). Culture is also expressed at the community level where the community is characterized by norms associated with their culture (Linkenbach \& Otto, 2014).

Ethnic Identity - A multifaceted and dynamic construct reflecting the sense of culture, group membership and setting that can be conceptualized at an individual and collective perspective (Phinney \& Ong, 2007). Effects of ethnic identity may or may not be internalized the same way as an individual or as a group, or across members that make up the group. 
Ethnically Diverse Black Women - This term represents the ethnic variation among Black women who live in the target community and were eligible to participate in this study. Ethnicities represented in this study included African American, Haitian, Jamaican, Bahamian, Panamanian, American Indian, South African, and European. Health Perceptions - Perceptions reference the "thought processes" that the women in this study "go through before taking a health-related action" (Edberg, 2007, p.35). These are specifically the women's ideas, beliefs, and attitudes toward breast self-exams and having a mammogram as influenced by their family, social networks, culture, prior experiences with health representatives, and exposure to health information or the media. Health-Seeking Behaviors - This dependent variable includes two separate behaviors (a) seeing a doctor for a preventive visit, and (b) seeing a doctor related to a concern about screening for cancer (breast, cervical, colon).

Heterogeneity - Considers the national identity of Black residents living in Broward who participated in this study in addition to the racial identity of "Black." This provides an opportunity for participants to "self-identify" their group membership (i.e., African American, Jamaican, and Bahamian) to "capture more robust information" to complement measures of "nativity." (NA, p.24) Inclusion of this variable addresses the potential "differences immigrants have in self-perceptions and knowledge about racial definitions that differ from those in the United States". (US Census, p.24)

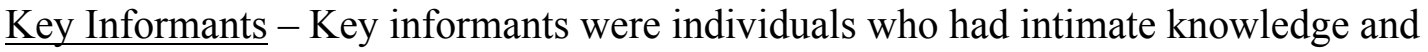
expertise of the social, political, or cultural aspects of the 33311 community, its residents or population at-large. Their knowledge informed the progression of the study from conceptualization to interpretation of the study. Informants held one of four roles in this 
study: (1) consultant prior to development of the research tools and conceptualization of the methodology, (2) served as members of a community advisory board created to guide the implementation of the study and interpretation of study data, (3) were enrolled from the 33311 community and the immediate vicinity as study participants, or (4) were volunteers from the community who assisted with study implementation through support and in-kind services. Study participants were the only mutually exclusive informant category.

Neighborhood Organization Participation - One of four forms of social capital and “refers to residents' formally organized collective activity for addressing neighborhood issues" (Carpiano, 2007).

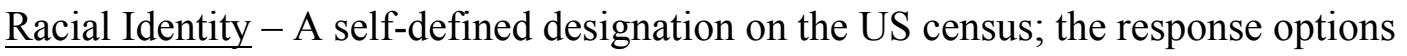
"adhere to the 1997 Office of Management and Budget (OMB) standards on race and ethnicity which guide the Census Bureau in classifying written responses to the race question": Individuals for the 2000 and 2010 census were provided five options (White, Black or African American, American Indian or Alaska Native, Asian, and Native Hawaiian or other Pacific Islander). "The racial categories included in the census questionnaire generally reflect a social definition of race recognized in this country and not an attempt to define race biologically, anthropologically, or genetically. In addition, it is recognized that the categories of the race item include racial and national origin or sociocultural groups. People may choose to report more than one race to indicate their racial mixture, such as "American Indian" and "White." People who identify their origin as Hispanic, Latino, or Spanish may be of any race.” Race. (n.d.). Retrieved July 20, 2016 from http://www.census.gov/topics/population/race/about.html. 


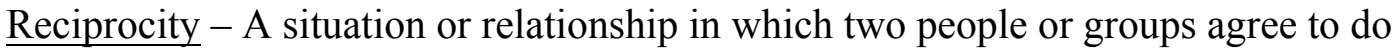
something similar for each other, to allow each other to have the same rights, etc. a reciprocal arrangement or relationship. Reciprocity. (n.d.). Retrieved July 20, 2016, from http://www.merriam-webster.com/dictionary/reciprocity

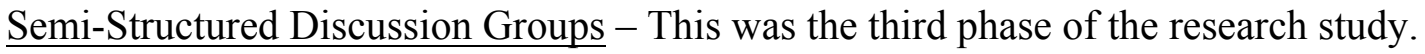
Semi-structured discussions followed the administration of the survey. Semi-setructured discussions were focus groups in which a semi-structured interview guide was used by the moderator to lead the discussion.

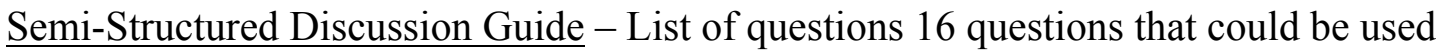
during the discussion groups. Questions were arranged in four clusters by topic. Moderator had the flexibility in the administration of these questions ask or omit questions from the list based upon the flow of the conversation.

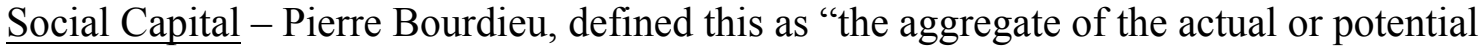
resources which are linked to possession of a durable network of more or less institutionalized relationships of mutual acquaintance or recognition" (Bourdieu, 1985, p.248).

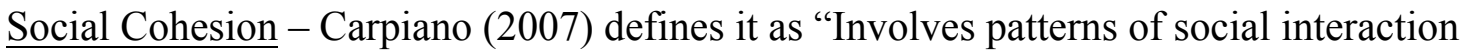
and values (e.g., network formation and ties, familiarity, and mutual trust). It serves as the foundation from which social capital can be formed."

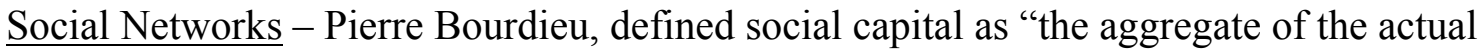
or potential resources which are linked to possession of a durable network of more or less institutionalized relationships of mutual acquaintance or recognition" 
(Bourdieu, 1985, p. 248; Bourdieu, 1980). Within social capital the networks "allow individuals in a community to claim access to resources possessed by their associates" (Portes, 1998, p. 4). Membership to the networks conveys importance because the "social relations within the networks translate into action and confer potential benefits and harms" (Carpiano, 2007, p). 641, for the residents.

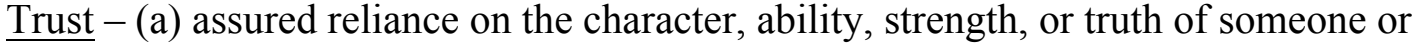
something, (b) one in which confidence is placed, (c) dependence on something future or contingent-hope. Trust. (n.d.). Retrieved July 20, 2016, from http://www.merriamwebster.com/dictionary/trust

\section{Summary}

In summary, the disproportionately high rates of mortality for Black women related to breast cancer are a consistent problem in Broward County. This problem is possibly mediated by: (a) the fact that so many of the Black women present with this disease in its later stages, and (b) the ethnic diversity found among Black women who live in this county. Broward is not alone, for the disproportionate number of Black women who die from breast cancer is a national concern for public health and it is a problem that remains unresolved. The goal then of this study is to understand, from the perspective of Black women that live in a community adversely impacted by this trend, why they are not obtaining mammograms in a more timely fashion. This dissertation used both semi-structured discussion groups and a self-administered survey to see if ethnic identity, culture, and social cohesion would explain this phenomenon. Chapter 1 summarized the need for this study, the questions that have been addressed by this 
research, and the limitations of this study. Chapter 2 will provide more detail of the

literature and social context of the community where the study was conducted.

\section{LIST OF REFERENCES}

Airhihenbuwa, C.O. \& Liburd, L. (2006). Eliminating health disparities in the African American population: the interface of culture, gender and power. Health Education Behavior, 33, 488-501.

Akinyemiju, T. F., Soliman, A. S., Johnson, N. J., Altekruse, S. F., Welch, K., Banerjee, M., ... Merajver, S. (2013). Individual and Neighborhood Socioeconomic Status and Healthcare Resources in Relation to Black-White Breast Cancer Survival Disparities. Journal of Cancer Epidemiology, 472-490. http://doi.org/10.1155/2013/490472

Allen, J.D., Mars, D.R., Tom, L. et al. (2013). Health beliefs, attitudes and service utilization among Haitians. Journal of Health Care Poor \& Underserved, 24(1), 106-119. doi: 10.1353/hpu.2013.0015.

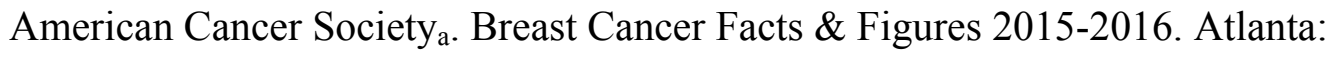
American Cancer Society, Inc., 2015.

American Cancer Society . Cancer Facts \& Figures for African Americans 2016-2018. Atlanta: American Cancer Society, 2016.

American Cancer Society, 2016. American Cancer Society recommendations for early breast cancer detection in women without breast symptoms. Retrieved from http://www.cancer.org/cancer/breastcancer/moreinformation/breastcancerearlydete ction/breast-cancer-early-detection-acs-recs

Bailey, E. J., Erwin, D. O., \& Belin, P. (2000). Using cultural beliefs and patterns to improve mammography utilization among African-American women: the Witness Project. Journal of the National Medical Association, 92(3), 136-142.

Bourdieu, P. (1980). Lecapitalsocial:notesprovisoires. ActesRech.Sci.Societ, 31, 2-3.

Bourdieu, P. (1985). The forms of capital. In Handbook of Theory and Research for the Sociology of Education, (ed.) JG Richardson, pp. 241-58. NewYork:Greenwood

Brondolo, E., Gallo, L.C. \& Myers, H.F. J. (2009). Race, racism and health: disparities, mechanisms, and interventions. Behavioral Medicine, 32 (1), 1-8. doi:10.1007/s10865-008-91903

Brown, C. R., Hambleton, I. R., Hercules, S. M., Alvarado, M., Unwin, N., Murphy, M. M., ... Younger-Coleman, N. (2017). Social determinants of breast cancer in the 
Caribbean: a systematic review. International Journal for Equity in Health, 16, 60. http://doi.org/10.1186/s12939-017-0540-z

Carpiano, R. M. (2006). Toward a neighborhood resource-based theory of social capital for health: Can Bourdieu and sociology help? Social Science \& Medicine, 62, $165-175$.

Carpiano, R.M. (2007). Neighborhood social capital and adult health: An empirical test of a Bourdieu-based model. Health \& Place, 13(3), 639-655.

CDC (2013) SMART BRFSS City and County Data, (PHSIPO). Retrieved December 15, 2013 http://apps.nccd.cdc.gov/BRFSS-

SMART/SelQuestion.asp?MMSA=All\&yr2=2011\&VarRepost=\&cat=WH\#WH

Consedine, N.S. (2012). The Demographic, system, and psychosocial origins of mammographic screening disparities: Prediction of initiation versus maintenance screening among immigrant and non-immigrant women. Journal of Immigrant Minority Health, 14(4), 570-582. doi:10.1007/s10903-011-9524-Z

Danforth, D. N. (2013). Disparities in breast cancer outcomes between Caucasian and African American women: a model for describing the relationship of biological and nonbiological factors. Breast Cancer Research, 15(3), 208. http://doi.org/10.1186/bcr3429

DeSantis, C. E., Fedewa, S. A., Goding Sauer, A., Kramer, J. L., Smith, R. A. \& Jemal, A. (2016). Breast Cancer Statistics, 2015: Convergence of Incidence Rates Between Black and White Women. Cancer: A Cancer Journal for Clinicians, 66 (1), 31-42. doi: 10.3322/caac.21320

DeSantis, C. E., Lin,C. C., Mariotto, A. B., Siegel, R. L., Stein, K. D., Kramer, J. L., ... \& Jemal, A. (2014). Cancer Treatment and Survivorship Statistics, 2014. Cancer: A Cancer Journal for Clinicians, 64 (4), 252-271. doi: 10.3322/caac.21235

Edberg, M. (2007) Essentials of Health Behavior Social and Behavioral Theory in Public Health, Richard Riegelman (Series Ed.), Sundbury MA:Jones and Bartlett Publisher, Inc.

Ehrlich, T. (Ed.). (2000). Civic Responsibility and Higher Education. (pp. Preface-xi). Phoenix, AZ:Oryx Press, 2000.

Erwin, D. O., Spatz, T. S., Stotts, R. C. and Hollenberg, J. A. (1999), Increasing Mammography Practice by African American Women. Cancer Practice, 7, 78-85. doi:10.1046/j.1523-5394.1999.07204.x 
Erwin, D., Spatz, T.S., Stotts, R.C., Hollenberg, J.A., \& Deloney, L.A.(1996). Increasing mammography and breast self-examination in African American women using the witness project model. Journal Cancer Education, 11(4), 210-215. http:// doi.org/10.1080/08858199609528430

Florida Department of Health Bureau of Epidemiology Chronic Disease Epidemiology Section, Division of Disease Control and Health Protection. Report of the Florida Behavioral Risk Factor Surveillance System (BRFSS) 2013 Data, Broward. Retrieved from http://www.floridahealth.gov/statistics-and-data/surveydata/behavioral-risk-factor-surveillancesystem/reports/ documents/2013county/ documents/Broward.pdf

Gierisch, J.M., O’ Neill, S. C., Rimer, B. K., DeFrank, J. T., Bowling, M. \& Skinner, C. S. (2009). Factors associated with annual-interval mammography for women in their 40s. Cancer Epidemiology, 33(1), 72-78. http:// doi.org/10.1016/j.cdp.2009.03.001

Grills, C., \& Longshore, D. (1996). Africentrism: psychometric analyses of a self-report measure. Journal of Black Psychology, 2(2), 86-106.

Hankerson, G., Council of Elders (2003). Across the Tracks. Fort Lauderdale, FL:Broward County Library Association.

Hughes, C., Lerman, C., \& Lustbader, E. (1996). Ethnic differences in risk perception among women at increased risk for breast cancer. Breast Cancer Research and Treatment, 40, 25-35.

Jackson, J. S., Neighbors, H. W., Torres, M., Martin, L. A., Williams, D. R. \& Baser, R. (2010). Use of Mental Health Services and Subjective Satisfaction With Treatment Among Black Caribbean Immigrants: Results From the National Survey of American Life. American Journal of Public Health, 97 (1), 60-67.

Jones, C., Maben, J., Jack R. H., Davies, E. A., Forbes, L., Lucas, G. \& Ream E. (2014). A systematic review of barriers to early presentation and diagnosis with breast cancer among black women. BMJ Open, 4(2), e004076. http://doi.org/10.1136/bmjopen-2013-004076

Jones, N. A. \& Johnson, T. D. (2012). The Black Population: 2010 [PowerPoint]. Retrieved from https://www.census.gov/newsroom/releases/pdf/2012-0201 black slides.pdf

Kawachi, I., Kennedy, B.P. \& Glass, R. (1999). Social Capital and self-rated health: A contextual analysis. American Journal Public Health, 89(8), 1187-1193.

Kobetz, E. \& Parker, D. Cancer Data for South Florida: A Tool for Identifying Communities in Need, Report to the Health Foundation of South Florida. 
Disparities and Community Outreach Core, University of Miami Sylvester Comprehensive Cancer Center; 2010. Research Funded by Health Foundation of South Florida and community-based organizations.

Lannin, D.R., Mathews, H.F., Mitchell, J., Swanson, M.S. (2002). Impacting cultural attitudes in African-American women to decrease breast cancer mortality. The American Journal of Surgery, 184, 418-423.

Laurence, J. (2011). The effect of ethnic diversity and community disadvantage on social cohesion: A multi-level analysis of social capital and interethnic relations in UK communities. European Sociological Review, 27(1), 70-89.

Linkenbach, L. \& Otto, L. (2014). Promoting Positive Community Norms. A Supplement the CDC's Essentials for Childhood: Steps to Create Safe, Stable, Nurturing Relationships and Environments. Retrieved from Division of Violence Prevention at the Centers for Disease Control and Prevention, Atlanta, GA. https://www.cdc.gov/violenceprevention/pdf/efc-promoting-positive-communitynorms.pdf.pdf

Lipkus, I.M., Iden, I. M., Terrenoire, J. \& Feaganes, J. R. (1999). Relationships among breast cancer concern, risk perceptions, and interest in genetic testing for breast cancer susceptibility among African-American women with and without a family history of breast cancer. Cancer Epidemiology Biomarkers \& Prevention, 8(6), 533-539.

McQueen, A., Vernon, S.W., Rothman, A. .J, Norman, G.J., Myers, R.E. \& Tilley, B.C. (2010). Examining the role of perceived susceptibility on colorectal cancer screening intention and behavior. Behavioral Medicine, 40, 205-217.

Musgrave, C. F., Allen, C. E., \& Allen, G. J. (2002). Spirituality and health for women of color. American Journal of Public Health, 92(4), 557-60. PMCID: PMC1447116

National Academies (1996). Spotlight on Heterogeneity, The Federal Standards for Racial and Ethnic Classification, Summary of a Workshop by National Research Council Committee on National Statistics. Presented by Barry Edmonston, Juanita Tamayo Lott, Joshua Goldstein of the National Research Council (U.S.). Retrieved from

https://books.google.com/books?id=Oj8rAAAAYAAJ\&printsec=frontcover\&sour $\mathrm{ce}=\mathrm{gbs}$ ge summary $\mathrm{r} \& \mathrm{cad}=0 \# \mathrm{v}=$ onepage $\& \mathrm{q} \& \mathrm{f}=$ false

O'Donnell, C. R. and Tharp, R. G. (2012). Integrating cultural community psychology: Activity settings and the shared meanings of intersubjectivity. American Journal of Community Psychology, 49, 22-30. doi:10.1007/s10464-011-9434-1 
O’Neal, C. W., Wickrama, K. A. S., Ralston, P. A., Ilich, J. Z., Harris, C. M., Coccia, C., ... Lemacks, J. (2014). Health insurance status, psychological processes, and older African Americans' use of preventive care. Journal of Health Psychology, 19(4), 491-502. http://doi.org/10.1177/1359105312474911

Osypuk, T. L. (2013). Future Research Directions for Understanding Neighborhood Contributions to Health Disparities. Revue D'epidemiologie et de Sante Publique, 61(2), S61-S68. http://doi.org/10.1016/j.respe.2013.03.040

Padamsee, T. J., Wills, C. E., Yee, L. D., \& Paskett, E. D. (2017). Decision making for breast cancer prevention among women at elevated risk. Breast Cancer Research, 19, 34. http://doi.org/10.1186/s13058-017-0826-5

Patel, K., Kanu, M., Liu, J., Bond, B., Brown, E., Williams, E., ... Hargreaves, M. (2014). Factors influencing Breast Cancer Screening in Low-Income African Americans in Tennessee. Journal of Community Health, 39(5), 943-950. http://doi.org/10.1007/s10900-014-9834-X

Peek, M.E., Sayad, J.V. \& Markwardt, R. (2008). Fear, fatalism and breast cancer screening in low-income African-American women: the role of clinicians and the health care system. Journal General Internal Medicine, 23(11), 1847-53. doi: 10.1007/s11606-008-0756-0. Epub 2008 Aug 27

Phinney, J.S. (1992). The Multigroup Ethnic Identity Measure: a new scale for use with diverse groups. Journal of Adolescent Research, 7, 156-176.

Phinney, J.S. \& Ong, A.D. (2007). Conceptualization and measurement of ethnic identity: current status and future directions. Journal of Counseling Psychology, 54(3):271-281.

Poonawalla, I., Goyal, S., Mehrotra, N., Allicock, M., Balasubramanian, B.A. (2014). Attitudes of South Asian women to breast health and breast cancer screening: Findings from a community based sample in the United States. Asian Pacific Journal of Cancer Prevention, 15(20), 8719-8724.

Portes, A. (1998). Social Capital: Its Origins and Applications in Modern Sociology. Annual Review of Sociology, 24(1), 1-24 DOI:10.1146/annurev.soc.24.1.1.

Race. (n.d.). In Miriam-Webster's Learner's Dictionary. Retrieved July 20, 2016 from http://www.census.gov/topics/population/race/about.html.

Ray, B. (2003). The role of cities in immigrant integration. Migration Policy Institute, Washington, DC. Retrieved April 18, 2017 from http://www.migrationpolicy.org/article/role-cities-immigrant-integration 
Reciprocity. (n.d.). In Miriam-Webster's Learner's Dictionary. Retrieved July 20, 2016, from http://www.merriam-webster.com/dictionary/reciprocity

Rice, L. J. \& Halbert, C. H. (2017). Chapter Five - Social Networks across Common Cancer Types: The Evidence, Gaps, and Areas of Potential Impact. Advances in Cancer Research, 133, 95-128. doi: 10.1016/bs.acr.2016.09.002

Russell, K. M., Champion, V. L., Monahan, P. O., Millon-Underwood, S., Zhao, Q., Spacey, N., ... Paskett, E. D. (2010). Randomized Trial of A Lay Health Advisor and Computer Intervention to Increase Mammography Screening in African American Women. Cancer Epidemiology, Biomarkers \& Prevention : A Publication of the American Association for Cancer Research, Cosponsored by the American Society of Preventive Oncology, 19(1), 201. http://doi.org/10.1158/1055-9965.EPI-09-0569

Schmitz, K. H., Neuhouser, M. L., Agurs-Collins, T., Zanetti, K. A., Cadmus-Bertram, L., Dean, L. T., \& Drake, B. F. (2013). Impact of Obesity on Cancer Survivorship and the Potential Relevance of Race and Ethnicity. JNCI Journal of the National Cancer Institute, 105(18), 1344-1354. http://doi.org/10.1093/jnci/djt223

Scott, J. (Ed.) (2015). Dictionary of Sociology (4 ed.). Retrieved from http://www.oxfordreference.com.ezproxy.fiu.edu/view/10.1093/acref/9780199683 581.001.0001/acref-9780199683581-e-2113? rskey $=$ saAfts\&result $=2$

Sellers, R.M., Rowley, S.A., Chavous, T.M., Shelton, J.N. \& Smith, M.A. (1997). Multidimensional Inventory of Black Identity: a preliminary investigation of reliability and construct validity. Journal of Personal Social Psychology, 73, 805815.

Short, S. E., Yang, Y. C., \& Jenkins, T. M. (2013). Sex, Gender, Genetics, and Health. American Journal of Public Health, 103(0 1), 10.2105/AJPH.2013.301229. http://doi.org/10.2105/AJPH.2013.301229

Sussner, W.M., Edwards, T.A., Thompson, H.S., et al. (2011). Ethnic, racial and cultural identity and perceived benefits and barriers related to genetic testing for breast cancer among at-risk women of African descent in New York City. Public Health Genomics, 14, 356-370.

Trust. (n.d.). In Miriam -Webster's Learner's Dictionary. Retrieved July 20, 2016, from http://www.merriam-webster.com/dictionary/trust

Urban League of Broward County, (2013).Closing the Gap. The State of Black Broward: Health Report. Retreived from ULbroaward.org August 8, 2016.

US Census, 2014a. 2010-2014 American Community Survey 5-Year Estimates. Selected characteristics of the native and foreign born populations for broward county. U.S. 
Census Bureau, 2010-2014 American Community Survey 5-Year Estimates. Retrieved from

http://factfinder.census.gov/faces/tableservices/jsf/pages/productview.xhtml?src=C $\mathrm{F}$

US Census, 2014b. 2010-2014 American Community Survey 5-Year Estimates. Selected characteristics of the native and foreign born populations for 33311. U.S. Census Bureau, 2010-2014 American Community Survey 5-Year Estimates. Retrieved from

http://factfinder.census.gov/faces/tableservices/jsf/pages/productview.xhtml?src=C $\underline{F}$

Ward, S.H., Lin, K., Meyer, B., et al. (2008).Increasing colorectal cancer screening among African Americans, linking risk perception to interventions targeting patients, communities and clinicians. Journal of the National Medical Association, 100(6), 748-758.

Williams, D. R., Mohammed, S. A., Leavell, J., \& Collins, C. (2010). Race, Socioeconomic Status and Health: Complexities, Ongoing Challenges and Research Opportunities. Annals of the New York Academy of Sciences, 1186, 69101. http://doi.org/10.1111/j.1749-6632.2009.05339.x

Work, D. (2001). My Soul is a Witness: A History of Black Fort Lauderdale. Virginia Beach, VA:Donning Company Publishers. 


\section{CHAPTER II. REVIEW OF THE LITERATURE}

\section{Literature, Background, and Setting}

Black women have the highest mortality rates associated with breast cancer and are most likely to present late with advanced stages of this disease (ACS, 2015; DeSantis et al. 2016). Recent studies have explored the possibility of ethnic variation among Black women for these outcomes (Rice \& Halbert, 2017; Sussner et al., 2010). While the approach and study site may differ, the goal of this dissertation has in common with these studies the goal of drilling deeper into understanding whether the contributing factors for mortality are the same across the various ethnicities of women of African descent who live in the study site. Researchers have begun to appreciate that norms associated with country of origin may vary among Black people who live in the US (Molina \& James, 2016; Tsai et al., 2017; Stewart \& London, 2014). As a result, understanding relevant within-group variations in key behavioral factors among Black women may explain risk and mortality for Black women in the US. The following studies offer examples:

One study investigated possible ethnic variations in tumor characteristics and environmental exposures that may have led to the development of breast cancer (Camacho-Rivera, Kalwar, Sanmugarajah, Shapira \& Taioli, 2014). This study included women who reported being black without Caribbean ancestry (African Americans) and Black women with Caribbean ancestry (Caribbean) from Brooklyn, NY. Their study found statistically significant differences in breast cancer survival associated with ethnicity. Specifically, Black women with estrogen receptor negative tumors and progesterone negative receptive tumors, who were born outside of the US, experienced a significantly worse survival than women born in the US (Camacho-Rivera, Kalwar, 
Sanmugarajah, Shapira \& Taioli, 2014). Likewise, for women born outside the US, mainly in the Caribbean, their tumors were biologically more aggressive than the same size and age-matched tumors in the sample born in the US (Camacho-Rivera, Kalwar, Sanmugarajah, Shapira \& Taioli, 2014).

Another study explored patterns of mammography screening among immigrant and nonimmigrant women to establish predictors for adherence to mammography guidelines (Consedine, 2012). This study included African American, English Caribbean, Haitian, Dominican, Eastern European, and European American women from Long Island and Brooklyn, NY. Yet another study addressed a woman's nativity to understand the relationship between early life experiences and risk factors for breast cancer among Black women in Brooklyn (Borrell, Castor, Conway \& Terry, 2006). Women were stratified as US-born Black and other Black ethnic groups (Caribbean, Latin American and continental African-birthplace).

These selected studies highlight that race and ethnicity are being appreciated as powerful factors to address breast cancer screening among Black women. However, this work is concentrated in a limited region of the US, primarily the northeast; the scope of this research is broad and lacks replication. Also, the subpopulations are so varied that, as with heterogeneity between US- and foreign-born women, ethnic differences within members of the foreign-born populations may exist. This then sets the stage for pursuing research of diverse populations of Black women in South Florida.

\section{Process to Identify Relevant Literature}

Three procedures were used to identify relevant literature for this project. First, computerized databases were searched, including PubMED (PMC) and Dissertation 
Abstracts, using the key words: "ethnic identity," "cultural identity," "racial identity," "health disparities," "breast cancer," "cancer," and "social cohesion". Because researchers also use the terms "ethnicity," "race," and "culture," these terms were added to the searches. The original search initiated to develop this study was restricted to articles published between 2000 and 2013. An additional search was conducted to identify citations of three seminal reports that guided the conceptualization of this research: Carpiano (2006), Sussner and colleagues (2010), and the Urban League of Broward (2013). Carpiano's research (2007) introduced "social cohesion" as an essential element for communities to develop the capacity to address health issues within a neighborhood. The desire to examine the influence of cultural, ethnic, and racial identity on breast screening behavior and attitudes originated from the work of Sussner and colleagues (2010), who found that self-identity influenced behavior and knowledge regarding genetic testing for breast and cervical cancer among ethnically diverse Black women in New York. The Urban League report (2010) was published after passage of Affordable Care Act legislation and offered a comprehensive review of the various social and economic factors that contribute to health equity. This report highlights the extensive disparity of ZIP-code 33311. The area has a diabetic rate more than twice as high as the county rate and an unhealthy food index at well above the county rate (Urban League, 2010, p. 20). PubMed (PMC) was consulted for citations and other relevant articles.

Second, local newspapers, books, and oral histories that documented the history of Black residents in the target community of Broward County were reviewed. These materials were obtained from the African American Research Library and Cultural Center (AARL) and the Fort Lauderdale Historical Association. AARL provided access 
to a series of oral histories entitled "Trailblazers of Broward County," developed by Dr. Kitty Oliver; and two published histories of Blacks in Broward County entitled, "Across the Tracks" by Dr. Gwendolyn Hankerson with the Council of Elders and "My Soul is a Witness, A History of Black Fort Lauderdale" by Deborah Work, and the circulation of three local papers. Newspaper clippings were accessed from the vaults at the Historical Society.

Lastly, electronic databases available on the Internet from the US Bureau of the Census and other governmental agencies were searched. The American Factfinder website was the primary source used to obtain community-level information regarding demographic data on the residents and the target community. Terms used in the search included race, ethnicity, gender, nativity, age, residence, and language spoken at home, income, education and ZIP code. The website for the State of Florida Department of Health, Fort Lauderdale Office, was also accessed to obtain statistics on breast cancer, mammography utilization in Broward County, and reports released by the department on their breast screening programs.

\section{The Problem of Breast Cancer Screening in the Community \\ Breast cancer among Black women nationally and South Florida.}

Breast cancer continues to be the second-most prevalent cancer among all women, following skin cancer, in the US. In one year, an estimated 123,840 women will have been diagnosed with breast cancer. Of these cases, 40,290 women will have died from this disease (ACS, 2015; DeSantis et al., 2016). Recent data indicate that incidence rates over the past five years have been decreasing among white women while, during the same period, the rates for Black women continue to increase (ACS, 2015). Meanwhile, 
Black women continue to have the highest mortality rates among all racial and ethnic groups, with Black women living in the Southern region of the US having the highest rates (ACS, 2015; DeSantis et al., 2015). Moreover, Black women continue to be diagnosed at later stages, have the greatest likelihood of developing the most aggressive forms of breast cancer, and have the lowest survival rates among all ethnicities (ACS, 2015, DeSantis et al., 2016).

The disparity between Black and white women for breast cancer is an ongoing health concern for Florida, with a greater challenge faced by Black women who live in South Florida. White women and women of African descent together comprise the largest segment of all breast cancer cases in Florida; however, this burden is not evenly distributed between the two ethnicities (ACS, 2016). Within south Florida, from 20102012, the incidence rate for breast cancer between Black and white women in Broward County was 1.9:1, suggesting that Black women in Broward were almost twice as likely to develop breast cancer than white women (Florida Charts, 2016). Likewise, the risk for Black women to develop this disease exceeds that for the state, where the incidence rate between Black women and white women is 1.4:1 (Florida Charts, 2016). Age-adjusted mortality data from 2012-2014 also show a similar pattern, although mortality rates between Black and white women indicate that Black women fair slightly better in Broward compared to the mortality rates for women of African descent statewide (1.2:1 in Broward versus 1.3:1 in Florida) (Florida Charts, 2016). The reasons why breast cancer risk remains higher for Black women have not been fully explicated.

Breast cancer risks for residential ZIP-code 33311. A disproportionate number of Black women who live in the target community (ZIP-code 33311) are diagnosed with 
late-stage breast cancer compared to those who live in other parts of Broward County. A report released by the Disparities \& Community Outreach Core in the Sylvester Cancer Center at the University of Miami (Kobetz \& Parker, 2010) reviewed reported breast, cervical, and colon cancer cases in three counties within South Florida (Miami-Dade, Broward, and Monroe) to identify community-level disparities in cancer mortality by ZIP-code. This study compared state and county cancer morbidity and mortality data extracted from the Florida Cancer Data System (FCDS), the state cancer registry (Kobetz \& Parker, 2010). The University of Miami report indicated that a higher proportion were diagnosed with late-stage breast cancer in the target community $(48.0 \%)$ compared to other women who lived in Broward (36.0\%), the state $(35.0 \%)$, and the national average (39\%) (Table1).

\begin{tabular}{|c|l|l|l|l|l|}
\hline \multicolumn{6}{|c|}{ Table 1. Cancer Profile from FCDS Geocodes by } \\
\hline & $\begin{array}{l}\text { 5-Yr Total } \\
\text { Cases and } \\
\text { Percentage } \\
\text { Diagnosed Late } \\
\text { in ZIP-code } \\
\text { Area 33311 }\end{array}$ & $\begin{array}{l}\text { 5-Yr Total } \\
\text { Cases and } \\
\text { Percentage } \\
\text { Diagnosed Late } \\
\text { in Broward } \\
\text { County }\end{array}$ & $\begin{array}{l}\text { 5-Yr Total } \\
\text { Cases and Per. } \\
\text { Diagnosed } \\
\text { Late in State } \\
\text { of Florida }\end{array}$ & $\begin{array}{l}\text { Rates } \\
\text { Significantly } \\
\text { Higher in } \\
\text { Target } \\
\text { Community } \\
\text { than } \\
\text { County? }\end{array}$ & $\begin{array}{l}\text { Increase } \\
\text { from 1997- } \\
\mathbf{2 0 0 2}\end{array}$ \\
\hline Breast & $123(48 \%)$ & $5,323(36 \%)$ & $60,901(35 \%)$ & Yes & Yes \\
\hline Cervical & $24(54 \%)$ & $441(53 \%)$ & $3,945(51 \%)$ & No & Yes \\
\hline Colorectal & $138(57 \%)$ & $3,889(59 \%)$ & $44,885(57 \%)$ & No & No \\
\hline
\end{tabular}

Source: Kobetz \& Parker, 2010

\section{Underlying Factors that Contribute to Breast Cancer Mortality in the Community}

Screening adherence and risk for breast cancer. Underlying the high rates of mortality for Black women are their low adherence rates to screening recommendations for having a mammogram (ACS, 2015). Early and consistent participation in breast 
screening is recommended to address breast cancer for it (a) reduces the rate of women that are diagnosed with this disease in its later stages and (b) increases women's opportunities for survival (ACS, 2016). Consequently, many of these women will only receive care after many of their available options have become ineffective due to the delay in diagnosis. Despite the availability of programs such as the Florida Breast and Cervical Cancer Early Detection Program (FLBCCEDP) that provides assistance to lowincome, uninsured, and underserved women to access mammograms, a large number of eligible Black women who live in Broward are still diagnosed late with this disease. Likewise, many Black women do not receive mammograms. During 2013, only 45.1\% $(25.9 \%-64.4 \%, \mathrm{p} \leq 0.05)$ of Black women $40-76$ years of age self-reported completing a mammogram within the past 12 months compared to $53.4 \%(42.5 \%-64.3 \%, \mathrm{p} \leq 0.05)$ of the white women in the same cohort (Florida Department of Health, 2013). The reasons why Black women do not take more advantage of these programs that have been successful in other states is unclear.

\section{Individual-, structural-, and environmental-level factors. Several} sociodemographic factors contribute to Black women not being adherent to breast cancer screening recommendations (i.e., lack of medical insurance and lower educational attainment). Several individual-level factors influence the health-seeking behaviors of Black women and they include: a lack of understanding about the disease and perception of risk, along with a woman's perception about her health; fear about the exam; nativity, and overall attitudes about the utility of medical procedures and screening (vis-à-vis fatalism, trust and benefit) (Lannin, Matthews, Mitchell \& Swanson, 2002; Guidry, Matthews-Juarez \& Copeland, 2003; Jackson et al., 2007; Beckjord \& Klassen, 2008; 
Peek, Sayad \& Markwardt, 2008; Gierisch et al., 2009; Jones et al., 2014). Additionally, women living in ethnically diverse communities similar to Broward often do not follow screening guidelines due to a complex, multi-level set of barriers that exist influencing Black women's perception of cancer risk for developing cancer and using cancer screening (Ward et al., 2008; Griva et al., 2009; McQueen et al., 2010; Nadarzynski et al, 2012; Marcus et al., 2013; Allen et al., 2013). Therefore, any investigation that limits its examination to individual-level factors will not effectively address the complexities found in the issues that influence Black women's participation in breast cancer screening and involvement in the US health care system.

Cancer screening interventions have been criticized because they tend to focus on individual-level determinants, absent consideration of other social and systemic factors that have been associated with unhealthy outcomes that exist among poor or disenfranchised populations (Pasick \& Burke, 2008). Among these are a woman's insurance status and their accessibility to medical and screening services which have been critical structural barriers to women's health behaviors (O’Neal et al., 2014; Jones et al., 2014). Other structural factors include whether the women are part of a network that promotes healthier behaviors (i.e., sharing information, resources, emotional and psychological support) and length of time being part of the community influence the health behaviors of African-American and Caribbean women (Berkman \& Glass, 2001; Griffith, Johnson, Zhang, Neighbors \& Jackson, 2011).

The disproportionate risk of Blacks involves a host of systemic factors, such as lower socioeconomic status, high unemployment and underemployment, lower educational attainment, high rates of economic consumerism coupled with low rates of 
vested ownership in economic and social structures, history of medical mistrust and abuse, health attitudes, cancer beliefs, community resources, faith, and institutional racism (Andersen \& Hill-Collins, 1997; Coburn, 2000; Lynch, 2000; Purnell et al., 2010; Brittain et al, 2012; Sajid et al., 2012). Moreover, non-adherence to screening recommendations within the 33311 ZIP-code area occurs as part of a broader, long-term issue of poorer health outcomes for the residents.

Report Black Broward (2010) illustrates that Black people, especially, in ZIPcode 33311, face several health issues simultaneously. Health issues range for HIV/AIDS, chronic conditions, mental health, and comorbidities within an environment where unemployment and underemployment, lower educational attainment, and limited access to nutritional resources prevail. Many factors interplay with women's ability to engage with Broward's health care system. As a result, the report suggests that (a) the issue of non-compliance occurs in an environment where poorer health is normalized by the residents and stakeholders and (b) poor health is linked to the overarching issue of residents not receiving primary or preventive care services. Therefore, understanding how these factors influence low participation in cancer screening among Black women is necessary as a foundation to eliminate this disparity in Broward.

Current cancer screening practice. As has been mentioned, the most effective means to reduce cancer mortality is to encourage individuals to be screened early and for them to remain compliant with screening recommendations after an initial exam (ACS, 2016). Broward County has adopted a National Breast and Cervical Cancer Early Detection Program (NBCCEDP) to improve women's breast cancer screening rates (FL Department of Health, 2015). This model was created by the Centers for Disease Control 
and Prevention (CDC) to assist low-income, uninsured, and underserved women who need access to breast and cervical cancer screening and diagnostic services (Miller, Plescia \& Ekwueme, 2014). While Broward County enrolled over 1,200 women in this program from 2010 to 2012 (Broward Department of Health, 2013), data reported by Kobetz and Parker (2010) suggest that many of the women who are developing breast cancer may not enroll in this program or are enrolling too late to benefit from early and frequent screening. Furthermore, the national program has successfully served approximately 4.4 million women in the past 20 years, but the barriers faced by Black women in Broward may be more complex and multi-layered than in other areas (Broward Department of Health, 2013; Levano et al, 2014).

\section{Description of Residents and Featured Study Community}

Demographic characteristics of women living in ZIP-code 33311. Since the Sylvester Cancer Center 2009 study, the target community has remained predominately of African descent; slightly more women over 18 years of age than men live in the target community and in the rest of Broward (53.3\% and 52.3\% respectively). More households were headed by women in the target community (30.4\%) as compared to Broward (15.0\%). The median age was 39.7 years for women living in the target community compared with 34.1 years of age for the remainder of Broward. More families living in the target community (35.1\%) made less than $\$ 25,000$ per year compared with $15.9 \%$ for Broward County. One-fifth (21.1\%) of those 65 years old and older who lived in the target area had incomes below the Federal poverty level compared with $12.2 \%$ that lived elsewhere in Broward. Just over one quarter (26.3\%) of the women 
18-64 years of age in this community were living under the poverty level compared with $12.2 \%$ in Broward (Table 2).

\begin{tabular}{|c|c|c|c|c|c|c|c|c|c|c|}
\hline \multicolumn{11}{|c|}{$\begin{array}{c}\text { Table 2. Black Women Living in Target Community Compared to Broward } \\
\text { Source: US Census Bureau, American Fact Finder 2008-2012 American Community } \\
\text { Survey 5-Year Estimates }\end{array}$} \\
\hline Setting & $\begin{array}{l}\text { ‘08-'12 } \\
\text { Total Pop } \\
\text { Est }(16+)\end{array}$ & $\begin{array}{c}\% \\
\text { Black } \\
\text {-not } \\
\text { Hispa } \\
\text { nic } \\
(\%)\end{array}$ & $\begin{array}{l}\text { Wome } \\
\text { n } 18+ \\
(\%)\end{array}$ & $\begin{array}{l}\text { Wome } \\
\mathrm{n} 65+ \\
(\%)\end{array}$ & $\begin{array}{c}\text { Househol } \\
\text { ds }\end{array}$ & $\begin{array}{l}\text { Av } \\
\text { Hous } \\
\text { e Size }\end{array}$ & $\begin{array}{l}\text { Women } \\
\text { Employ } \\
\text { ed } 16+ \\
(\%)\end{array}$ & $\begin{array}{l}\text { Media } \\
\mathrm{n} \\
\text { Income }\end{array}$ & $\begin{array}{l}\text { Incom } \\
\text { e } \\
<\text { Pover } \\
\text { ty } 18- \\
64(\%)\end{array}$ & $\begin{array}{l}\text { Income } \\
<\text { Povert } \\
\text { y } 65+ \\
(\%)\end{array}$ \\
\hline 33311 & 47,630 & 83.5 & 53.3 & 57.8 & 20,641 & 3.03 & 50.4 & $\begin{array}{l}30 \\
744\end{array}$ & 26.3 & 21.1 \\
\hline Broward & $1,418,498$ & 25.8 & 52.3 & 26.7 & 665,913 & 3.33 & 56.0 & 51,603 & 12.2 & 12.5 \\
\hline
\end{tabular}

Ethnic diversity of Black women in ZIP-code 33311. Almost $78 \%$ of the residents living in the target community were born in the US compared to $65.7 \%$ in Broward County. In both the target community and Broward, almost half of those foreign born (22.9 v. 34.9\%) were women (53.8 v. 53.9\%). Of those who are foreign born, more in the target community (94.8\%) originated from regions that represent the Caribbean compared to other parts of Broward County (78.5\%). Likewise, almost $23 \%$ of the residents in the target community self-identified as West Indian compared to $13.5 \%$ in Broward County. Approximately $20 \%$ in the target community spoke a language other than English within their homes compared to $37.5 \%$ in Broward County (Table 3). 


\begin{tabular}{|l} 
Table 3. Demographic Distribution in Target Community by Ethnicity and Nativity \\
Source: US Census Bureau, American Fact Finder 2008-2012 American Community Survey 5-Year \\
Estimates \\
\hline Setting
\end{tabular}

Geographical boundaries of target community. The target community, ZIP-

code 33311 , contains 63,786 men, women, and children, according to the 2010 US Census (2014). This ZIP-code is comprised of five residential areas and includes the City of Fort Lauderdale to its south and east, Wilton Manors to its north and east, Lauderhill and Lauderdale Lakes to its west, and central city which is surrounded by the other neighborhoods. The community is a mix of residential and sole proprietorship; the majority of residents are renters $(33,599)$ compared to homeowners $(29,135)$. Each of these separate segments has its own unique characteristics. In the northwest corner is an established West Indian community, to the northeast border an affluent and predominately white community, and surrounding the lower east rim is a flourishing, gentrified community. Within the target area, however, the residents find few modern amenities, with off-brand gas stations, road side BBQ establishments and independently- 
owned restaurants, a well-known open vegetable stand, established cemeteries, and a newly opened Bank of America. This study sought to enroll Black women who lived primarily in the central, northwest, and lower central sections of the targeted area. The residents who lived in the omitted areas of the study site were predominately not of African descent and thus did not represent the populations of interest nor fit the selection criteria.

Fiqure 3. Map of Residential ZIP-code 33311

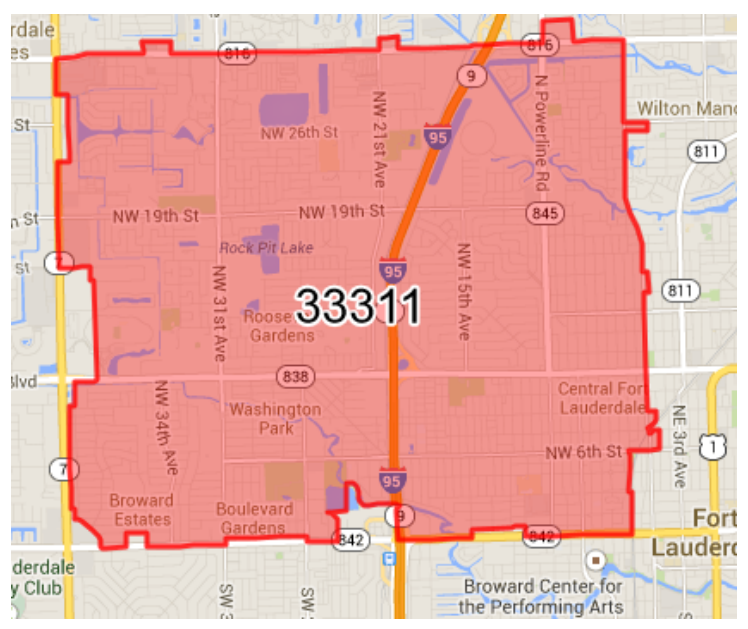

\section{Social Cohesion and Women's Health}

Importance of social capital. Social capital is broadly seen as the various levels of social relationships formed through social networks ability of actors to secure benefits by virtue of membership in social networks and other social structures" (Portes, 1998, p. 6). Thus, by belonging to social networks, individuals can secure certain benefits and resources that would not be possible in the absence of these networks. Eriksson (2011) explains that members of the community draw upon internalized norms, group solidarity, and reciprocity among members to create social support, social influence, social control 
and social participation among members to develop backing and trust regarding a central issue of the network or community. These characteristics move individuals along a pathway where individuals: (a) are motivated to create access to resources or deny others access to resources that foster health in the community, (b) through modeling demonstrate behavior that is healthy or destructive to one's health status, (c) receive status from association with or exclusion from the network, and (d) obtain cognitive skills, gratification, or a sense of belonging by being involved in the network. Collectively, these characteristics act as incentives, influence health behavior, and increase an individual's social capital cache in the community (Coleman, 1988). Given the theoretical relevance of social capital to a population's health outcomes, this dissertation will explore three components of the model developed by Carpiano (2006), social cohesion, connectedness, and values, to potentially explain the difficulty of Black women to adhere to screening recommendations in this community.

Relationship of social capital and social cohesion to health. Social cohesion is an important component of social capital and is defined as patterns of social interaction and values (such as network formation and ties, familiarity, and mutual trust) that lead to social capital, and which serve as intermediaries between structural antecedents and social capital, but are necessary foundations for establishing social capital within neighborhoods (Carpiano, 2006). There are three distinct qualities to social cohesion that manifest at both the individual level and at the group level. Eriksson (2011) points out that just as individuals have the capacity to bond, bridge, and connect together, creating a common network around a particular issue, so too do the networks have the capacity to bond, bridge and connect with each other in a community around a health-related issue. 
Social cohesion exists across these networks to the extent that the functions of bonding, bridging and connecting occur. Specifically, to bond means that individuals connect and trust each other to form a network. To bridge means that across networks, mutual trust, support, norms of reciprocity, and norms of solidarity occur so that residents can work toward a common priority in their community. Finally, to connect means that diverse networks work together to obtain resources outside of their community for a common need.

African-American and Afro-Caribbean women living in ZIP-code 33311 would benefit from these connections through their involvement in situations that create social support, social integration, reciprocity, and civil society (Szreter and Woolcock, 2003). Therefore, this dissertation is concerned with the likelihood that ethnic identity and culture could be used to facilitate cohesion within and across African-American and Caribbean women. If so, then the relationship between both groups to work together would suggest an opportunity to bridge the populations for a common goal to prioritize better health-seeking behaviors in their community.

Social capital conceptual framework literature review. The conceptual framework selected for this study was adapted from Carpiano's (1997) model of social capital (Figure 1). His model illustrates various pathways that lead to health outcomes within modern, urban, communities. The major components found among these pathways include individual-level traits among the residents, social cohesion within a community, the values and connections that create cohesion in the communities, and the presence of social capital in the community. Carpiano's model also accounts for the influence of 
external, structural-level, factors that act upon a community's ability to promote or seek healthier alternatives.

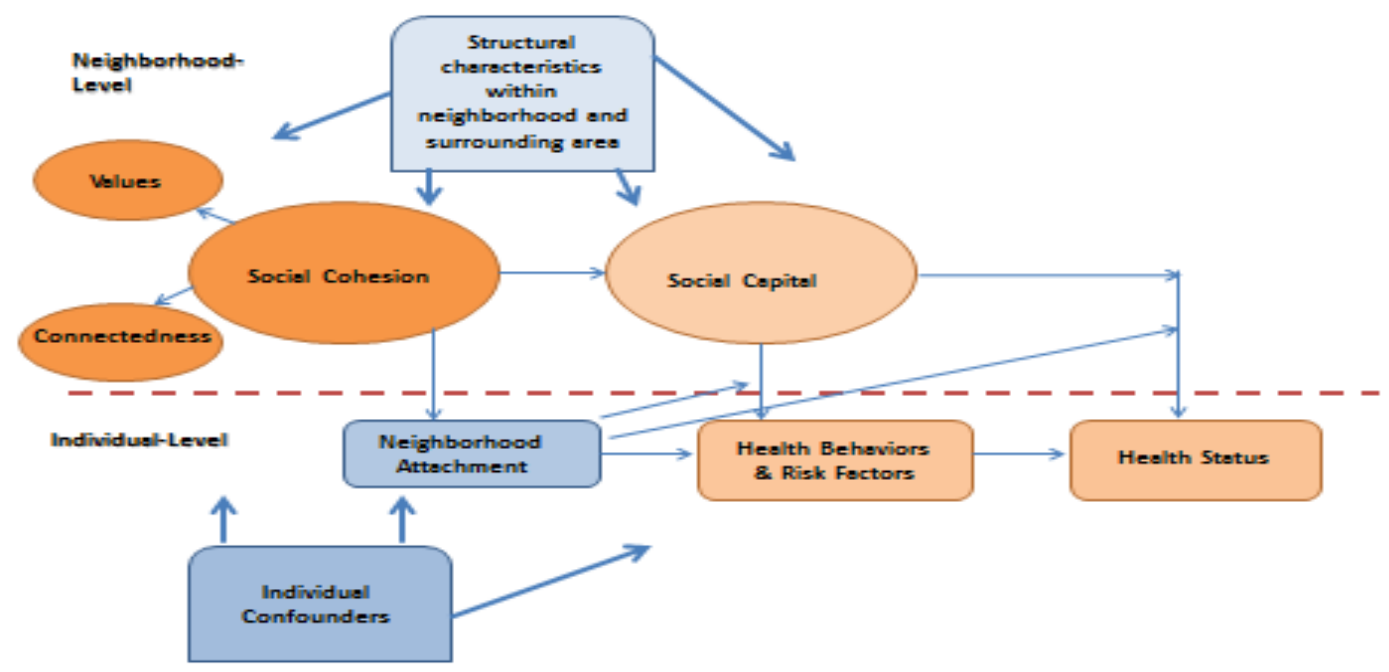

Figure 4. Structured Characteristics, Social Cohesion, and Social Capital: Carpiano's Conceptual Model

\section{Analytical Framework}

The scope of this dissertation's research limited its exploration of Carpiano's model to three constructs: 1) social cohesion, 2) values, and 3) connectedness, and their influence on the health status of residents within this community (Carpiano, 2007) (Figure 3). Modifications were made to the constructs that represented values and connectedness.

The construct "culture" was used to represent the social connections that often exist among Black women because of commonalities in cultural beliefs, values, and norms in particular groups or the community where they live (Jones et al., 2014; Egede, 2006). Common connections created by culture can lead to perception of cohesiveness between Black women who mutually share common cultural beliefs, norms, and values 
(Melvin et al., 2016; Klassen \& Washington, 2008). The same culturally moderated interactions dictate the thinking and actions of Black women individually and the norms adopted in their communities to engage in health behaviors (Likenbach \& Otto, 2014; Klassen \& Washington, 2008).

Connections are also created among Black women due to commonalities in their personal or familial ethnic identity. Ethnic identity, like cultural identity, can promote cohesion among Black women who, on some level, connect related to their ethnicities (Dean et al., 2014). Likewise, culture and ethnic identity are interrelated while having an independent influence on perceived cohesion among Blacks older adults who live in urban, residentially segregated neighborhoods (Armstrong-Brown, Eng, Hammond, Zimmer \& Bowling, 2015; Sussner et al., 2011). Modifications have been made accordingly to the conceptual model (Figure 4).

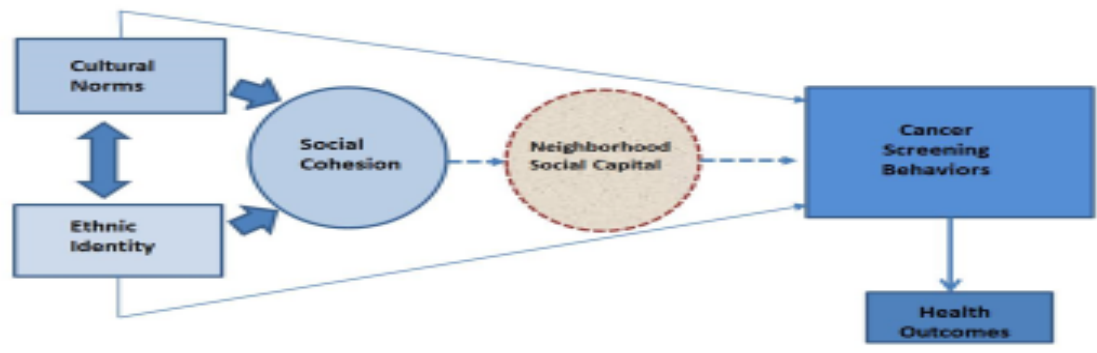


Ethnic identity, connectedness and Black women. In view of the importance placed by Afro-Caribbean and African-American women on ethnicity to define trust, interpersonal attitudes, and familiarity in social interactions, ethnic identity best represents the concept connectedness for Black women (Jaynes, 2005; Branch \& Young, 2006). The differences in social experiences and expectations among African Americans and Afro-Caribbean immigrants in the US have dictated the capacity of the two groups to coexist cohesively in a community (Jaynes, 2005; Shaw-Taylor \& Tuch, 2007; Branch \& Young, 2006). In the mid-1960's the United States reduced its restrictions on individuals who could emigrate from the Caribbean to this country. Given the social environment of this time and the dominance of segregation in this country, African Americans and AfroCaribbean immigrants had to live in the same communities and found themselves in direct competition with each other for employment opportunities that were limited. While African Americans and the new immigrants both originated from Africa, their migrations patterns from Africa created two cultures and, over time, destroyed their perception of a common legacy among the descendants. Their relationships since have been characterized by misunderstandings, stereotypes, friction, and at times, described as contentious (Jaynes, 2005; Shaw-Taylor \& Tuch, 2007; Branch \& Young, 2006). As a result, Caribbean and African Americans historically have avoided each other socially and maintain separate existences within the same communities, yet, maintain separate enclaves where both groups prioritize individual identities, cultural ties, culture, and social characteristics (Jaynes, 2005).

Essential to the establishment of social cohesion is the ability of neighbors to identify with each other, find common ground, and establish trust (Kawachi, Kennedy \& 
Glass, 1999). Therefore, in ethnically diverse communities like ZIP-Code 33311, ideas such as the contact hypothesis argue that the ability for the two groups to establish close and sustained contact with each other requires an interaction that promotes the development of positive attitudes toward an opposite group (Jaynes, 2005). The fact that ethnic enclaves remain a vested part in communities such as this one, works against the likelihood of developing cohesiveness between the two groups. Laurence (2013) explains that in diverse communities contact among ethnically diverse neighbors moderates the negative effects of diversity improving conditions yet the effects are further moderated by levels of disadvantage in the community (Laurence, 2013). As such, low levels of contact and the large number of the women that are within the lower income level may challenge the residents to enthusiastically pursue cohesive ties that bond, bridge and link in their community. Consequently, an investigation into the likelihood that African American and Caribbean women could find common ground despite their potential differences based upon their culture and ethnicity is warranted.

Ethnic identity can also be viewed as a multifaceted and dynamic construct reflecting the sense of culture, group membership, and setting that can be conceptualized at an individual and collective perspective (Phinney \& Ong, 2007). Phinney (1990) explains that ethnic identity contains several characteristics that include selfidentification, sense of belonging to the group, perceived similarity, salience, commitment, social participation, and cultural practices. Likewise, Phinney and Ong (1997) argue that the effects of culture and ethnic identity may not be internalized the same way as an individual, as a group, or across members that make up the group; nor does culture remain static over time. 
Culture, norms, and Black women. Culture shapes the values adopted by Black women and the subsequent beliefs influence their health behaviors. Culture is defined as "the learned and shared beliefs, values, and life ways of a designated or particular group which are generally transmitted intergenerationally and influence one's thinking and action modes" (as cited in Egede, 2006, p. 668). Culture is also expressed at the community level where the community is characterized by actual and perceived norms associated with their culture (Linkenbach \& Otto, 2014). For this dissertation, it has been assumed that the behavioral norms adopted by the women and the broader community to engage with screening and healthcare services result from the overarching values, beliefs, and attitudes that have been shaped by their culture (Linkenbach \& Otto, 2014). Likewise, women's perceived norms, whether of the individual or of the collective body of residents, influence behaviors and community priorities.

Following the above rationale, it is also expected that culture provides a contextual reference for a woman that guides her motivation, rationalization, and prioritization to seek opportunities for a mammogram and complete the exam. Beyond the issue of cost, sociocultural concerns and beliefs often become the primary influence on the uptake of a mammogram by Black women (Jones et al., 2014; Lannin, 2002). Therefore, identifying the specific cultural characteristics and their context for the community towards breast cancer and the exam is imperative if one plans to make a substantial improvement in the screening behavior of the women (Hoffman-Goetz \& Mills, 1997; Pasick \& Burke, 2008).

Hence, factors such as self-efficacy, perceived cancer risk, anticipated pain from the exam, and knowledge about breast cancer and the exam are viable obstacles for 
women participating in the exam. Added to this list are issues of faith as culture, social support, cultural identity, cultural empowerment, and expectations (Airhehenbuwa \& Webster, 2004). While these factors have been found to influence the behavior of Black women, more evidence is needed to determine if their influence has the same significance when heterogeneity and nativity are also considered. Some of these factors are known but the inclusion of culture as a prominent underlying domain is a novel approach to address health disparities among Black women. Given that almost one-fourth of the community in this study represents women who self-describe as Caribbean and are foreign-born, such examinations are necessary.

The inclusion of culture as a prominent independent variable is based upon the research of Sussner and colleagues (2010). They studied the influence of cultural variations among ethnically diverse Black women regarding their perceived benefits and barriers related to genetic testing for breast and cervical cancers. Their cultural identity was African-American centered (Africentrism) and lacked some relevance for women who had emigrated from the Caribbean. Other studies have found that culture influences health-seeking behaviors of Black women, although these have been conducted in primarily African-American samples (Shinagawa, 2000; Dushay et al., 2001; Lannin et al., 2002). Additionally, emerging evidence is finding that factors such nativity, temporal orientation, medical mistrust, spirituality, and fatalism are important when investigating Black women's participation in mammography (Donovan and Tucker, 2000; Lannin et al., 2002; Peters, et al., 2005; Ford et al., 2007; Peek et al. 2008; Gullatte et al., 2010). Those studies that have used ethnically diverse populations have been limited in their geographic location to primarily the northeastern region of the US or overseas (Karlsen, 
2004; Arthur \& Katkin, 2006; Sussner et al., 20011). Expanding explorations of ethnic identity, culture, nativity and heterogeneity in areas such as South Florida where certain ethnicities have not been examined is needed. Moreover, since this area of research is newer, replication and confirmatory stories are also needed.

Social networks as component of social cohesion. Social networks are important in establishing a cohesive community. Networks typically are comprised of people who may or may not be familiar with each other, but it is the social structure that binds these individuals together in a community (Kritsotakisa \& Gamarnikow, 2003). Networks can have access to or create resources that can be used to improve health (Lochner, Kawachi, Brennan \& Buka, 2003). Network members often define health problems, facilitate the location of the services, and can influence the use of community resources toward health programming (Lin, 1999; Kawachi \& Berkman, 2000; Kawachi, 2006). These factors, in turn, lead to the creation of neighborhood social capital. (Carpiano, 2006, 2007).

Within many urban social environments, there are several pathways by which social networks can strengthen the relationship between social cohesion and a community's health status. First, the presence of strong social bonds may promote the diffusion of knowledge about health-related innovations (e.g., breast and cervical cancer screening), and through modelling or association, improve the target health behaviors (Erwin et al., 2006; Kim et al., 2006). Second, drawing on evidence related to low collective efficacy within a neighborhood to address cancer screening, social cohesion may help to generate informal social control over health-related behaviors, and possibly facilitate collective action among residents to ensure access to local services and 
amenities that may be relevant to health (Erwin, 2006). Third, through psychosocial processes, social cohesion may serve as a source of affective support and mutual respect, and strengthen qualities such as mutual trust among neighbors or other psychosocial resources at the individual level, which in turn may directly, or indirectly, improve mental and physical health (Kim \& Kawachi, 2011). These opportunities have encouraged this research to suggest avenues that social networks may be promoted within the community to encourage cohesion and greater uptake of breast cancer screening.

\section{Historical Accounts of Cohesion}

\section{Cohesion among African-American and Afro-Caribbean populations.}

Osborne (2000) explains that race, ethnicity, and nativity have created divisions among Blacks in the United States. Black immigrants have recognized the critical and inferior position that African Americans occupy compared to most whites living in the US. The social divisions that occur between African Americans and Black Caribbeans have been attributed to various negative stereotypes that are depicted about African Americans through the media, literature, and racist propaganda that historically confront people of African descent before arriving in the US (Jaynes, 2005). The vestiges of slavery were different for African Americans compared to other Blacks whereby post emancipation resulted in Blacks being able to advance socially and assume leadership roles which did not typically occur among Blacks in the US (Jaynes, 2005). The differences in these experiences have had a lasting impact on the ability of both groups to find common ground, and in some cases, non-American Black women may make conscious decisions to disassociate themselves from African American women and perceive it necessary to 
assert their cultural and national differences to succeed in this country (Portes \& Stepick, 1993; Osborne, 2000; Butterfield, 2001).

Black American women, conversely, expect allegiance from Black Caribbean women because of common racial and ancestral backgrounds. However, Reid (1970), Zepher (1996), Portes \& Stepick (1994) and Butterfield (2001) have explained that the historical experiences of Blacks enslaved in the US differ greatly from those in other countries and the American interpretations for the concepts "race" and "ethnicity" have created a different context for racial and ethnic identity than assumed by African Americans. The result is an environment having a propensity for misunderstanding, competition, and embedded hostility between African Americans and people of African descent who have emigrated from the Caribbean, Africa, or European countries (Osborne, 2000; Butterfield, 2001).

Cohesion within residential Zip-code 33311. Work (2001) and Hankerson (2003) developed two detailed accounts on the historical experience of Black residents in Broward County tracing their experiences from late $19^{\text {th }}$ century. These books describe the social networks that promote community cohesion, the external factors that influenced the social and health conditions of Black residents, and strategies adopted by community members to promote health, wellbeing, survival and growth for Black populations living in the community and surrounding segregated Black communities. Cohesive social networks that once offered stability, security, and opportunity for the target community may have diminished over time. Early Black settlers migrated from northern areas of Florida, the Bahamas, and the surrounding southern states to live and earn income in what was once called "Colored Town." Colored Town ran from north and 
south in Broward County extending in the northern most area of Deerfield and Pompano through central Broward to southern cities within Broward with Blacks living in Pompano, Deerfield, Dania, Carver Ranches, Hallandale and Ojus. The extension of Colored Town covered an area that permeated most of the formal areas of early Broward, but the Black communities located in Broward had limited access to the larger Broward and Fort Lauderdale areas because of the Jim Crow and segregationist practices that governed during this period (1900-1970's).

Central County, where the target community is located, offered many of the early and longstanding social, economic, medical and spiritual resources for all of Black Broward. The two publications on this community describe the culture of the community as one of trust, collective action, and pride for the community (Work, 200; Hankerson 2003). The residents represented resourceful, educated, blue-collar workers and field laborers; all living and worshipping side-by-side in a protective cluster. Until the 1960's Central County offered access to economic, social services, and social support that was created internally by and for other Black residents. To that extent, Central County played an important role in promoting a quality of life for all Blacks within these cities.

Black residents have remained for generations in the geographical boundaries of the target community and the broader Colored Town. The residents have experienced the adverse effects of slavery, racism, segregation, and social isolation that have been an underlying context for most in this community (Work, 2001; Hankerson, 2003). This context could influence members' sense of trust and willingness to secure outside assistance with community matters and ongoing trust with people from outside of their community network (Linn, 1999; Kawachi and Berkman, 2000; Kawachi, 2006). 
The target community has been a gateway for people emigrating from the Caribbean to establish themselves as residents in this country. This process has been met with resistance from African-Americans who have lived for several generations in the area. At issue has been perceived competition for jobs, awards, entitlements and respect from members of the dominant Anglo culture coupled with the influence of stereotypes and conflicting cultural characteristics (Osborne, 2001; Jackson \& Cothran, 2003). During the 1990's and early 2000's the growth of ethnic gangs became a major issue for the communities in Broward County. Their growth was in response to ethnic competition and hostility among local youth that was in response to ethnic and cultural differences between African-American and Caribbean groups (Jaynes, 2007). This culminated in 2001 with an interethnic summit to find common solutions as well as Broward County Schools taking action within locations with high West Indian student enrollment (Jaynes, 2007).

\section{Summary}

This chapter has presented information that is relevant to framing the disproportionate problems of mortality related to breast cancer and diagnosis of breast cancer in its later stages for Black women nationally and in the specific research site in Broward County. While research has been able to offer information that consistently explains the barriers faced by Black women to screen for breast cancer, less evidence is available that supports the integrity of this data for ethnically diverse Black women who live in the US. As a result this study will explore ethnic differences in attitudes, sociocultural factors, and health-seeking behavior in a diverse sample of AfricanAmerican and Afro-Caribbean women living in an area of Broward County, FL. 
Contextual considerations such as nativity, culture, and ethnic identity are newer lines of thought for most researchers related to health-related investigations of Black women. Greater consideration of these factors in warranted. This study has included one measure of nativity and three measures of ethnic identity in the cross-sectional survey. This study also dedicated a significant part of the semi-structured interviews to collect information from the women that identifies cultural characteristics and the meaning of culture as social context for their community and their self-described ethnicity. Social cohesion is an important characteristic of social capital, but a better understanding of the landscape of social interactions between Afro-Caribbean and African-American women is needed to suggest the likelihood that ethnic identity, culture and social cohesion will be effective in this community.

Based on this review of the literature, a study of breast cancer screening among African-American and Afro-Caribbean women 40 years of age and older living in ZIPcode 33311 will be conducted to address the following six questions:

\section{Quantitative}

1. What are the perceptions and health-seeking behaviors about cancer among ethnically diverse Black women (African-American and Afro-Caribbean)?

2. What is the relationship between ethnic identity and health-seeking behaviors among African-American and Afro-Caribbean women?

3. What is the relationship between ethnic identity and social cohesion among AfricanAmerican and Afro-Caribbean women? 


\section{Qualitative}

4. Are there differences in perceptions about cancer and mammograms between AfricanAmerican and Afro-Caribbean women?

5. How do ethnically diverse Black women characterize ethnic identity and culture?

6. How does ethnic identity and culture influence the health-seeking behaviors of ethnically diverse Black women?

Chapter 3 will provide an overview of the methods used in this study to answer these six questions.

\section{LIST OF REFERENCES}

Acevedo-Garcia, D., Bates, L. M., Osypuk, T. L., \& McArdle, N. (2010). The effect of immigrant generation and duration on self-rated health among US adults 20032007. Social Sciences and Medicine, 71, 1161-1172. doi:10.1016/j.socscimed.2010.05.034

Airhihenbuwa, C. O. (1995). Health and Culture Beyond the Western Paradigm. Thousand Oaks, CA:Sage Publications.

Airhihenbuwa, C.O. \& Liburd, L. (2006). Eliminating health disparities in the African American population: the interface of culture, gender and power. Health Education Behavior, 33, 488-501.

Allen, J.D., Mars, D.R., Tom, L., et al. (2013). Health beliefs, attitudes and service utilization among Haitians. Journal of Health Care Poor \& Underserved, 24(1), 106-119. doi: 10.1353/hpu.2013.0015.

Altschuler, A., Somkin, C. P., Adler, N. E. (2004). Local services and amenities, neighborhood social capital, and health. Social Science and Medicine 59, 12191229 http://dx.doi.org/10.1016/j.socscimed.2004.01.008

American Cancer Society (2013). Cancer Facts \& Figures for African Americans 20132014. Atlanta: American Cancer Society.

Andersen, M. L. \& Hill-Collins, P. (1997). Race, Class, and Gender: An Anthology (with InfoTrac) (The Wadsworth Sociology Reader Series), Belmont, CA:Wadsworth Publishing Co. 
Armstrong-Brown, J., Eng, E., Hammond, W. P., Zimmer, C., \& Bowling, J. M. (2015). Redefining Racial Residential Segregation and its Association With Physical Activity Among African Americans 50 years and Older: A Mixed Methods Approach. Journal of Aging and Physical Activity, 23(2), 237-246. http://doi.org/10.1123/japa.2013-0069

Beckjord,E. B. \& Klassen, A. C. (2008). Cultural values and secondary prevention of breast cancer in african american women. Cancer Control, 15(1), 63-71.

Berkman LF, Glass T. Social integration, social networks, social support, and health. In: Berkman LF, Kawachi I, editors. Social epidemiology. Oxford Press University:Oxford, UK; 2000. pp. 137-173.

Borrell, L.N., Castor, D., Conway, F. P. \& Terry, M. B. (2006). Influence f nativity status on breast cancer risk among US black women. Journal of Urban Health Bulletin of the New York Academy of Medicine, 83 (2), 211-220. doi:10.1007/s11524-0059014-5

Bowen, D., Hickman, K.M. \& Powers, D. (1997). Importance of psychological variables in understanding risk perceptions and breast cancer screening of African American women. Women's Health, 3(3-4), 227-242.

Branch, A. \& Young, R. (2006). Ethnic identity development of African Americans: Experiences in search of a paradigm. The Western Journal of Black Studies, 30(3), 160-170.

Brittain, K., Loveland-Cherry, C., Northhouse, L., Caldwell, C.H. \&Taylor, J.Y. (2012). Sociocultural differences and colorectal cancer screening among African American men and women. Oncology Nursing Forum 39(1), 100-107.

Brook, J.S. \& Pahl, K. (2005 The protective role of ethnic and racial identity and aspects of an Africentric orientation against drug use among African American young adults. Journal of Genetic Psychology, 166, 329-345.

Butterfield, S.P. (2001). Big tings a gwaan: Constructions of racial and ethnic identity among second-generation west indian immigrants. (Order No. 3016812, University of Michigan). ProQuest Dissertations and Theses, , 245-245 p. Retrieved from http://ezproxy.fiu.edu/login?url=http://search.proquest.com/docview/304708024?ac countid=10901.

Carlyse, S.K. (2012). Nativity differences in chronic health conditions between nationally representative samples of Asian American, Latino American, and Afro-Caribbean American respondents. Journal Immigrant Minority Health, 14(6), 903-911. 
Carpiano, R. M. (2006). Toward a neighborhood resource-based theory of social capital for health: Can Bourdieu and sociology help? Social Science \& Medicine, 62, 165175

Carpiano, R.M. (2007). Neighborhood social capital and adult health: An empirical test of a Bourdieu-based model. Health \& Place, 13(3), 639-655.

Coburn, D. (2000). Income inequality, social cohesion and the health status of populations: the role of neo-liberalism. Social Science \& Medicine, 51, 134-146.

Coleman, J.S. (1988). Social capital in the creation of human capital. American Journal of Sociology, 94 (Suppl), 95-120. http://www.jstor.org/stable/2780243

Coon, H. M. \& Kemmelmeier, M. (2001). Cultural Orientations in the United States. (Re)Examining Differences Among Ethnic Groups Journal of Cross-Cultural Psychology, 32(3), 348-364. doi: 10.1177/0022022101032003006 http://gsappweb.rutgers.edu/cstudents/readings/Summer/Summer/Kelly Diversity/ Coon $\% 202001 \% 20$ individualism $\% 20$ and $\% 20$ collectivism.pdf

Dean, L., Subramanian, S., Williams, D. R., Armstrong, K., Charles, C. Z., \& Kawachi, I. (2014). The Role of Social Capital in African-American Women's Use of Mammography. Social Science \& Medicine, 104, 148-156. http://doi.org/10.1016/j.socscimed.2013.11.057

DeSantis, C. E., Fedewa, S. A., Goding Sauer, A., Kramer, J. L., Smith, R. A. \& Jemal, A. (2016). Breast Cancer Statistics, 2015: Convergence of Incidence Rates Between Black and White Women. Cancer: A Cancer Journal for Clinicians, 66 (1), 31-42. doi: $10.3322 /$ caac. 21320

Donovan, K.A. \&Tucker, D.C. (2000). Knowledge about genetic risk for breast cancer and perceptions of genetic testing in a sociodemographically diverse sample. Journal Behavioral Medicine, 23(1), 15-36

Egede, L. E. (2006), Race, Ethnicity, Culture, and Disparities in Health care. Journal of General Internal Medicine, 21, 667-669. doi:10.1111/j.1525-1497.2006.0512.x

Eriksson, M. (2011). Social capital and health - implications for health promotion. Global Health Action, 4, 10.3402/gha.v4i0.5611. http://doi.org/10.3402/gha.v4i0.5611

Erwin, D.O., Johnson, V. A., Trevino, M., Duke, K., Feliciano, L. \& Jandorf L. (2007). A comparison of African American and Latina social networks as indicators for culturally tailoring a breast and cervical cancer education intervention. Cancer, 109 (2 Suppl), 368-377. 
Florida Department of Health (2015). Annual Legislative Report of the Florida Breast Cancer Early Detection and Treatment Referral Program. Retrieved from http://www.floridahealth.gov/diseases-and-conditions/cancer/breastcancer/ documents/bcc-2013-legislative-report.pdf

Ford, M.E., Alford, S.H., Britton, D., McClary, B. \& Gordon, H.S. (2007). Factors influencing perceptions of breast cancer genetic counseling among women in an urban health care system. Journal Genetic Counseling, 16, 735-753.

Fruchter, R. G., Wright, C., Habenstreit, B., Remy, J. C., Boyce, J. G. \& Imperato, P.J. (1985). Screening for cervical and breast cancer among Caribbean immigrants. Journal of Community Health, 10(3), 121-135.

Gilbert, K. L., Quinn, S. C., Goodman, R. M., Butler, J., \& Wallace, J. (2013). A MetaAnalysis of Social Capital and Health: A Case for Needed Research. Journal of Health Psychology, 18(11), 1385-1399. http://doi.org/10.1177/1359105311435983

Griffith, D. M., Johnson, J., Zhang, R., Neighbors, H. W., \& Jackson, J. S. (2011). Ethnicity, Nativity and the Health of American Blacks. Journal of Health Care for the Poor and Underserved, 22(1), 142-156. http://doi.org/10.1353/hpu.2011.0011

Griva F, Anagnostopoulos F, Madoglou S. (2009). Mammography screening and the theory of planned behavior: suggestions toward an extended model of prediction. Women Health. 49(8):662-681. doi: 10.1080/03630240903496010.

Gullatte MM, Brawley O, Kinney A, Powe B, Mooney K (2010). Religiosity, spirituality, and cancer fatalism beliefs on delay in breast cancer diagnosis in African American women. Journal of Religion and Health, 49(1):62-72. doi: 10.1007/s10943-0089232-8. Epub 2009 Jan 30

Hankerson, G., Council of Elders (2003). Across the Tracks. Fort Lauderdale, FL:Broward County Library Association.

Hoffman-Goetz, L. \& Mills, S.L. (1997). Cultural barriers to cancer screening among African American women: A critical review of the qualitative literature. Women's Health: Research on Gender, Behavior, and Policy, 3(3\&4), 183-201.

Jaynes, G. D. (2005). Encyclopedia of African American society. Thousand Oaks, Calif: Sage Publications.

Jones, C. E., Maben, J., Jack, R. H., Davies, E. A., Forbes, L. J., Lucas, G., \& Ream, E. (2014). A systematic review of barriers to early presentation and diagnosis with breast cancer among black women. BMJ Open, 4(2), e004076. http://doi.org/10.1136/bmjopen-2013-004076 
Kawachi, I., Kennedy, B.P. \& Glass, R. (1999). Social Capital and self-rated health: A contextual analysis. American Journal Public Health, 89(8), 1187-1193.

Kawachi, I., Subramanian, S. V. \& Kim, D. (2008). Social Capital and Health. New York, NY:Springer Science.

Kawachi I, Berkman L. (2000). Social cohesion, social capital, and health. In: Berkman LF, Kawachi I, eds. Social epidemiology. NY: Oxford University Press, p. 174-190.

Kawachi, I., Subramanian, S.V., \& Kim, D.(2008). Social capital and health: a decade of progress and beyond. In: Kawachi I, Subramanian SV, Kim D, eds. Social capital and health. New York, NY: Springer, pp. 126.

Kennedy, B.P., Kawachi, I., Prothrow-Stith, D., Lochner, K. \& Gupta, V. (1998). Social capital, income inequality, and firearm violent crime. Social Science Medicine, 47, 7-17.

Kim, D., Subramanian, S.V., \& Kawachi, I. (2008). Social capital and physical health: a systematic review of the literature. In: Kawachi I, Subramanian SV, Kim D, eds. Social capital and health. New York,NY: Springer, pp. 13990.

Kim, D., Subramanian, S.V. \& Kawachi, I. (2006).Bonding versus bridging social capital and their associations with self rated health: a multilevel analysis of 40 US communities. Journal Epidemiology Community Health, 60(2), 116-122.

Kim, D., Kawachi, I. (2008). Social Capital and Health: Edited By Ichiro Kawachi, S. V. Subramanian, and Daniel Kim. American Journal of Epidemiology, 168(11), 1340.

Kinket, B., \& Verkuyten, M. (1997). Levels of ethnic self-identification and social context. Social Psychology Quarterly, 60(4), 338-354. Retrieved from http://ezproxy.fiu.edu/login?url=http://search.proquest.com.ezproxy.fiu.edu/docvie $\mathrm{w} / 212710101$ ? accountid $=10901$

Kinney, A.Y., Croyle, R.T., Dudley, W.N., Bailey, C.A., Pelias, M.K. \& Neuhausen, S.L. (2001). Knowledge, attitudes, and interest in breast-ovarian cancer gene testing: a survey of a large African-American kindred with a BRCA1 mutation. Preventive Medicine, 33(6), 543-551

Kobetz, E. \& Parker, D. Cancer Data for South Florida: A Tool for Identifying Communities in Need, Report to the Health Foundation of South Florida. Disparities and Community Outreach Core, University of Miami Sylvester Comprehensive Cancer Center; 2010. Research Funded by Health Foundation of South Florida and community-based organizations. 
Kushner, H. I., \& Sterk, C. E. (2005). The limits of social capital: Durkheim, suicide, and social cohesion. American Journal of Public Health, 95(7), 1139-43. Retrieved from

http://ezproxy.fiu.edu/login?url=http://search.proquest.com.ezproxy.fiu.edu/docvie $\underline{\mathrm{w}} / 215098958$ ? accountid $=10901$

Lakey, B. amd Cohen, S. (2000). Social support theory and measurement In S. Cohen, L. G. Underwood and B. H. Gottlieb (Eds.). Social Support Measurement and Intervention: A Guide for Health and Social Scientists. Oxford University Press, Oxford:United Kingdom. (pp. 29-52).

Lannin, D.R., Mathews, H.F., Mitchell, J., Swanson, M.S. (2002). Impacting cultural attitudes in African-American women to decrease breast cancer mortality. The American Journal of Surgery, 184, 418-423.

Laurence, J. (2011). The effect of ethnic diversity and community disadvantage on social cohesion: A multi-level analysis of social capital and interethnic relations in UK communities. European Sociological Review, 27(1), 70-89.

Leininger MM (1985). Transcultural care diversity and universality: a theory of nursing. Nursing Health Care, 6(4), 208-212.

https://www.ncbi.nlm.nih.gov/pubmed/3846132

Levano, W., Miller, J. W., Leonard, B., Bellick, L., Crane, B. E., Kennedy, S. K., Haslage, N., ..., and Tharp, F. S. (2015). Public education and targeted outreach to underserved women through the National Breast and Cervical Cancer Early Detection Program. Cancer, 120 (16), 2591-2596. doi:10.1002/cncr.28819

Lochner, K. A., Kawachi, I., Brennan, R. T., Buka, S. L. (2003). Social capital and neighborhood mortality rates in Chicago. Social Science and Medicine, 56(8), 1797-1805. doi:10.1016/S0277-9536(02)00177-6

Mc Laughlin, L., \& Braun, K. (1998). Asian and Pacific Islander cultural values: Considerations for health care decision-making." Health and Social Work, 23 (2), 116-126. http://www.euromedinfo.eu/how-culture-influences-health-beliefs.html/

McQueen, A., Vernon, S.W., Rothman, A. .J, Norman, G.J., Myers, R.E. \& Tilley, B.C. (2010). Examining the role of perceived susceptibility on colorectal cancer screening intention and behavior. American. Behavioral Medicine, 40, 205-217.

Melvin, C. L., Jefferson, M. S., Rice, L. J., Cartmell, K. B., \& Halbert, C. H. (2016). Predictors of Participation in Mammography Screening among Non-Hispanic Black, Non-Hispanic White, and Hispanic Women. Frontiers in Public Health, 4, 188. http://doi.org/10.3389/fpubh.2016.00188 
Miller, J. W., Plescia, M. \& Ekwueme, D. U. (2014). Public health national approach to reducing breast and cervical cancer disparities. Cancer, 120 (16), 2537-2539. doi: $10.1002 /$ cncr. 28818

Moore, S., Haines, V., Hawe, P. \& Shiell, A. (2006). Lost in Translation: A geneology of the "social capital" concept in public health. Journal Epidemiology of Community Health,60, 729-734. doi: 10.1136/jech.2005.041848

Nadarzynski, T., Waller, J., Robb, K.A. \& Marlow, L.A. (2012). Perceived risk of cervical cancer among pre-screening age women (18-24 years): the impact of information about cervical cancer risk factors and the causal role of HPV. Sexually Transmitted Infection, 88(6), 400-406. Epub 2012 Apr 19

Nastas, D. (2012). Redefining culture from a psychological Perspective2. Psihologia Sociala, 30, 107-120. Retrieved from http://ezproxy.fiu.edu/login?url=http://search.proquest.com.ezproxy.fiu.edu/docvie w/1313208237? accountid=10901

O’Neal, C. W., Wickrama, K. A. S., Ralston, P. A., Ilich, J. Z., Harris, C. M., Coccia, C., Lemacks, J. (2014). Health insurance status, psychological processes, and older African Americans' use of preventive care. Journal of Health Psychology, 19(4), 491-502. http://doi.org/10.1177/1359105312474911

Osborne, B.L. (2000). Clash of identities in South Florida: An examination of the complexity of racial and national identity and their impact on ethnic solidarity between Black Americans and Jamaicans (Doctoral Dissertation, Florida International University). Retrieved from http://search.proquest.com/docview/304668492

Pasick, R. J. \& Burke, N. J. (2008). A critical review of theory in breast cancer screening promotion across cultures. Annual Review of Public Health, 29, 351-368.

Peek, M.E., Sayad, J.V. \& Markwardt, R. (2008). Fear, fatalism and breast cancer screening in low-income African-American women: the role of clinicians and the health care system. Journal General Internal Medicine, 23(11), 1847-53. doi: 10.1007/s11606-008-0756-0. Epub 2008 Aug 27.

Peters, N., Domchek S. M., Rose, A., Polis, R., Stopfer, J. \& Armstrong, K. (2005). Knowledge, Attitudes, and Utilization of BRCA1/2 Testing among Women with Early-Onset Breast Cancer. Genetic Testing, 9(1), 48-53. doi:10.1089/gte.2005.9.48

Phinney, J.S. \& Ong, A.D. (2007). Conceptualization and measurement of ethnic identity: current status and future directions. Journal of Counseling Psychology, 54(3):271281. 
Plescia, M., Wong, F. L., Pieters, J. \& Joseph, D. (2014). The National Breast and Cervical Cancer Early Detection Program in the era of health reform: A vision forward. Cancer, 120(16 suppl), 2620-2624. doi: 10.1002/cncr.28826

Portes A. (1998). Social capital: its origins and applications in modern sociology. Annual Review of Sociology, 24, 1-24.

Portes, A. \& Stepick, A. (1994). City on the edge: The transformation of Miami. Berkeley, Calif: University of California Press.

Purnell, J.Q., Katz, M.L., Andersen, B. A. \& Bennett, N. (2010). Social and cultural factors related to perceived colorectal cancer screening benefits and intentions in African Americans. Journal of Behavioral Medicine, 33(1), 24-34.

Molina, K. M., \& James, D. (2016). Discrimination, internalized racism, and depression: A comparative study of African American and Afro-Caribbean adults in the US. Group Processes \& Intergroup Relations : GPIR, 19(4), 439-461. http://doi.org/10.1177/1368430216641304

Reid, I.D.A. (1970). The Negro immigrant: His background, characteristics, and social adjustment, 1899-1937. New York:AMS Press.

Sajid, S., Kotwal, A.A. \& Dale, W. (2012). Interventions to improve decision making and reduce racial and ethnic disparities in the management of prostate cancer: A systemic review. Journal of General Internal Medicine, 27(8), 1068-1078.

Shaw-Taylor, Y. \& Tuchs, S. A. (2007). The Other African Americans. Contemporary African and Caribbean Immigrants in the United States. Lanham, MD:Roman \& Littlefield Publishers, Inc.

Shinagawa, S.M. (2000). The excess burden of breast carcinoma in minority and medically underserved communities. Cancer 88, (Suppl 5), 1217-1223. doi: 10.1002/(SICI)1097-0142(20000301)88:5+<1217::AID-CNCR7>3.0.CO;2-K

Stephens, C. (2008). Social capital in its place: Using social theory to understand social capital and inequalities in health. Social Sciences \& Medicine, 66, 1174-1184. doi:10.1016/j.socscimed.2007.11.026

Stewart, K. A. \& London, A. S. (2015). Falling through the cracks: Lack of health insurance among elderly foreign-born and native-born blacks. Journal of Immigrant and Minority Health, 17, 1391-1400. doi: 10.1007/s10903-014-0099-3

Sussner, W.M., Edwards, T.A., Thompson, H.S., et al. (2011). Ethnic, racial and cultural identity and perceived benefits and barriers related to genetic testing for breast 
cancer among at-risk women of African descent in New York City. Public Health Genomics, 14, 356-370.

Sussner, K. M., Thompson, H. S., Jandorf, L., Edwards, T. A., Forman, A., Brown, K., ... Valdimarsdottir, H. B. (2009). The influence of acculturation and breast cancerspecific distress on perceived barriers to genetic testing for breast cancer among women of African descent. Psycho-Oncology, 18(9), 945-955.

http://doi.org/10.1002/pon.1492

Tsai, H.-J., Surkan, P. J., Yu, S. M., Caruso, D., Hong, X., Bartell, T. R., ... Wang, X. (2017). Differential effects of stress and African ancestry on preterm birth and related traits among US born and immigrant Black mothers. Medicine, 96(5), e5899. http://doi.org/10.1097/MD.0000000000005899

Urban League of Broward County, (2013).Closing the Gap. The State of Black Broward: Health Report. Retreived from ULbroaward.org August 8, 2016.

US Census, 2014a. 2010-2014 American Community Survey 5-Year Estimates. Selected characteristics of the native and foreign born populations for broward county. U.S. Census Bureau, 2010-2014 American Community Survey 5-Year Estimates.

Retrieved from http://factfinder.census.gov/faces/tableservices/jsf/pages/productview.xhtml? src=C $\underline{F}$

US Census, 2014b. 2010-2014 American Community Survey 5-Year Estimates. Selected characteristics of the native and foreign born populations for 33311. U.S. Census Bureau, 2010-2014 American Community Survey 5-Year Estimates. Retrieved from http://factfinder.census.gov/faces/tableservices/jsf/pages/productview.xhtml? src=C $\underline{\mathrm{F}}$

Viruell-Fuentes, E. A., Miranda, P. Y. \& Abdulrahim, S. (2012). More than culture: Structuralracism, intersectionality theory, and immigrant health. Social Science \& Medicine, 75, 2099-2106 doi: 10.1016/j.socscimed.2011.12.037

Ward, S.H., Lin, K., Meyer, B., et al. (2008).Increasing colorectal cancer screening among African Americans, linking risk perception to interventions targeting patients, communities and clinicians. Journal of the National Medical Association, 100(6), 748-758.

Wolff, J. (1999). Cultural studies and the sociology of culture. Contemporary Sociology, 28 (5), 499-507 http://www.jstor.org/stable/2654982

Work, D. (2001). My Soul is a Witness: A History of Black Fort Lauderdale. Virginia Beach, VA:Donning Company Publishers. 
Zepher, F. (1996). Haitian Immigrants in Black America: A Sociological and Sociolinguistic Portrait. Westport, CT: Bergin and Garvey. 


\section{CHAPTER III. METHODS}

This chapter presents the methodology used to conduct a mixed-methods exploratory study consisting of three phases: Phase 1, creation of a community advisory board, Phase 2, administration of a cross-sectional survey to women who served as key informants from the community, and Phase 3, the conduct of a semi-structured discussion group with the same women. The chapter has been organized by the three study phases.

\section{Phase 1. Creation of a Community Advisory Board (CAB)}

Preliminary steps were taken to become familiarized with the community and to develop an orientation to the current social-, health-, and politically-related issues that impacted local residents. Several weeks were spent in the City of Fort Lauderdale's historical archives reviewing collected news clippings and researching documentation held at the African American Research Library and Cultural Center (AARL) that included relevant dissertations and books held in the special collections archives. All of these resources were specific to the history of the community. They included the experiences of Black people in their quest to become residents of the area and the various social and interpersonal dynamics that were part of the transition of various Black ethnicities to live in the community.

Key informants were first engaged in the study during this preliminary stage of the research. A list of key organizations and individuals was created based upon the research at the historical archives and AARL. A series of cold calls yielded five people who agreed to an in-person interview. These individuals provided their perspectives regarding the methodology and scope of the study, commentary on the history of the 
community, strategies for incorporating ethnically diverse participants, weighed in on the possible configuration of the $\mathrm{CAB}$, and suggested individuals for consideration in the CAB. Their suggestions included planning for the possibility that inclusion of all ethnicities would be difficult, the need to use a door-to-door approach to reach individuals, verified the AARL as a central venue for data collection activities, and suggested content for inclusion in the cross-sectional survey.

Eligibility to serve as a member of $\mathrm{CAB}$ was based upon the person: (1) a resident of the community, (2) a stakeholder in the health and welfare of the members of the community, (3) relevant expertise or affiliation with cancer/health organization, or (4) familiar with the community or the population. Efforts were made to create a $\mathrm{CAB}$ that reflected the demographics of the community and the inclusion criteria of this study; therefore, all but three $\mathrm{CAB}$ members were both 40 years of age or older and Caribbean or African American.

Starting with a community recommended health provider during the preliminary activities, snowball sampling methods were used to identify other potential candidates. Each person was interviewed and agreed to serve as a member and often recommend other candidates. $\mathrm{CAB}$ consisted of a total of 15 members with 10 people participating in the first face-to-face meeting where they provided comments on the proposed methods, data collection tools, and scope of the research. Five more members were added by the second face-to-face meeting to address gaps in expertise among the body identified by $\mathrm{CAB}$ members during the first face-to-face meeting.

Membership was defined as (1) commitment to two in-person meetings and one meeting by phone or some other electronic platform, (2) input on the data collection 
methods and materials, (3) support with the research activities, (4) feedback on intermediary data, and (5) facilitation with disseminating outcome findings into the community through a community meeting and local paper. As such, $\mathrm{CAB}$ members assumed the role of the second group of key informants to the study. Additionally, several volunteers provided support to activities through in-kind contributions for space to implement the discussion groups, advertising in community papers, dissemination of recruitment materials in the community, and support recruitment efforts by offering access to residents via donated radio airtime.

\section{Data Collection During Phase 2 and Phase 3}

Data from the study participants were collected during Phases 2 and 3. Phase 2, was the completion of a self-administered, cross-sectional survey and Phase 3 was the participation in a semi-structured discussion group. Phase 2 and Phase 3 were completed in the same two-hour time period with the study participants first completing the survey and, immediately following, participating in a discussion group. Post-hoc power analysis revealed that to achieve a medium effect size with two groups for analysis (African American versus Afro Caribbean) a minimum number of 108 surveys were needed. Thirty additional surveys were collected from women who were in attendance at AARL for sufficient power. These women met the same eligibility criteria in age, residency, and ethnicity but only completed the survey and did not participate in a discussion group (Figure 5). 


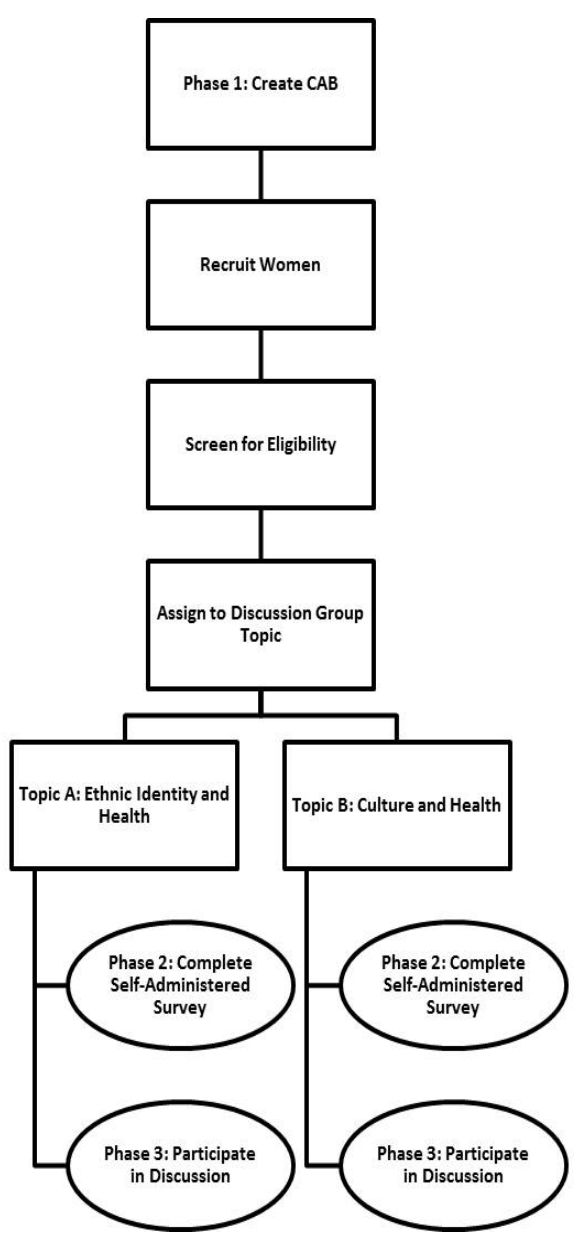

Figure 6. Order of Research Study Phases 1-3: Community Advisory Board, Administration of Self-Administered Survey, and Participation in Semi-Structured Discussion Group

\section{Activities that Facilitated Phase 2 and Phase 3}

Recruitment of study participants. A systematic process was followed to recruit the sample. The process incorporated three major steps: (1) construct a comprehensive list of key venues used by Black women within the community, (2) solicit participants through in-person contact while distributing study materials (fliers and posters) to individuals in the community, and (3) team with other community stakeholders (newspapers, local officials, CAB members, and volunteers) to expand the circle of 
access to eligible women via radio announcements, newspaper ads, and word of mouth. This list contained suggestions made by members of the study's CAB to identify additional areas within the community to recruit participants. CAB members suggested churches, nail, hair, and beauty salons, city agency offices, clinics, city parks, and a few restaurants. This researcher added laundromats and used the online Yellow Pages directory to identify all churches and salons within the community.

A stratified sample of nine out of 70 churches was drawn created using churches located in ZIP-code 33311 and the perimeter of the community. From a list created by public listings of churches, one church was randomly selected to represent each of the nine faith denominations (Catholic, Baptist, AME, Episcopal, Pentecostal, Methodist, Christian, Agape/Haitian, Other). Efforts were made to coordinate presentations and recruitment activities, church services, or other sponsored activities. Google Maps was used to identify the addresses of the salons and Laundromats within the community. Roads were selected from these maps based upon ease of access and density of these business locations. This researcher then walked the length of the major roads distributing study materials. In addition to CAB members, community stakeholders (i.e., local media and health ministry personnel) and other study participants provided suggestions for the recruitment list, actively disseminated research materials, and provided in-kind assistance with marketing this study in the community.

Study sample. A convenience sample of 121 women of African descent was recruited for this study. Each woman was 40 years of age and older, lived in or adjacent to the targeted ZIP code, self-identified racially as Black or mixed race and ethnically as African American or Caribbean (West Indian). Women who were foreign-born, spoke 
Haitian-Creole as their primary language, or had limited English literacy were included. Racial identity was included because the research inquiry was specific only to the issues and experiences of women of African descent who lived in or near this community. Age limitations were imposed to restrict participants to those women of average risk that met suggested age criteria by the American Cancer Society for participation in breast cancer screening (ACS, 2015). Residential restrictions were made to confine the geographical boundaries within the area of high prevalence. Four months into a projected eight month period residential restrictions were modified. Modifications enabled this researcher to advertise and recruit women from business and residences just across from the initial community perimeter. Accommodations were also made for language and literacy to allow participation from the full spectrum of Black women who lived in this community.

Protection of human subjects. This study protocol and procedures were approved by the Institutional Review Board (IRB) at Florida International University prior to data collection. Written informed consent was obtained on the day of the scheduled discussion group. Each participant also consented to have the group discussions recorded. To protect privacy, no identifying information was requested on the survey questionnaire. Names of participants were collected on sign-in sheets and consent forms.

\section{Phase 2: Administration of Self-Administered Surveys}

Study participants completed a cross-sectional survey following the consent process. One hour was reserved for the completion of Phase 2. Thirty additional women were recruited at the end of the data collection period to complete only the cross-sectional 
survey to increase statistical power for quantitative analyses. A total of 120 were enrolled into the study and 117 used in the quantitative analyses.

\section{Overview of Survey Content}

The self-administered survey (Appendix A) was designed to investigate the relationships of ethnic identity, racial identity, and social cohesion to the health perceptions and health-related behaviors of Black women. The domains of ethnic identity and racial identity were taken from the research tool created by Sussner and colleagues (2010) where they tested the relationship of ethnic, cultural, and racial identity to the perceptions and behaviors of Black women to genetic testing for breast cancer in New York City. The Multigroup Ethnic Identity Measure (MEIM) (Phinney, 1992) and the Other-Group Orientation Scale (Phinney, 1992) were used in the Sussner study to measure ethnic identity. Racial identity was measured by the Centrality subscale, one of four subscales that comprise the full Multidimensional Inventory of Black Identity (MIBI) (Sellers, Rowley, Chavous, Shelton \& Smith, 1997). The questionnaire replicates the approach of Sussner and colleagues (2010) to predict participation in genetic testing for breast and cervical testing among ethnically diverse Black women with a similar population, but addressing preventive medical behaviors and screening for cancer.

The questionnaire also included demographic items regarding participants' age and date of birth, residency characteristics, nativity, parental nativity, self-described race, self-described ethnicity, an estimate of previous year's annual income, educational attainment, insurance status, description of medical care point of contact, and summary of prior cancer screening history. Questions selected for the demographic section were to represent commonly used items that identify a population's characteristics (i.e., age, 
income, and education) and items that were thought to be appropriate for a component of the population who were multiethnic and foreign-born.(i.e., self-identified race, selfidentified race, nativity and parental nativity). Questions related to women's residency were included to document the stability of the residents within the target area and to suggest whether enough time had passed for residents who lived in close proximity to interact with each other. The demographic items were developed by the researcher.

The survey's remaining sections explored participant's health-seeking behaviors specific to completing a preventive medical exam and screening for cancer, perceived health status, awareness of regional breast and cervical cancer resources, perceived barriers, availability of health-related resources, perceptions toward mammograms and breast cancer, perceived risk for developing breast cancer, measures of social cohesion as an individual and member of a social group, and community-level social cohesion.

Unlike the Sussner study, this survey did not include measures of cultural identity in the cross-sectional survey. Instead, the discussion groups were used to document the women's perceptions and meaning of culture as specified by the community.

\section{Detailed Description of Each Survey Item.}

Ethnic identity. Ethnic Identity was calculated using both the Multi-Group Ethnic Identity Measure (MEIM) and the Other-Group Orientation Scale (OGO) both developed by Phinney (1992). These measures have been used in tandem to report ethnic identity with consistent internal reliability across various ethnic groups.

MEIM is composed of 12 questions as the full scale. It has been used to assess one's ethnic self-identification. MEIM has as its components two subscales: (1) ethnic identity achievement, herein, referred to as "ethnic search," and (2) affirmation and 
belonging, henceforth called "ethnic affirmation". Ethnic search mean score documents the extent to which a woman feels secure and confident in her ethnicity. This mean score comes from five questions within the full MEIM that independently ask the extent that she seeks information, is involved in social groups or organizations that include primarily their ethnic group, amount of thought given to the impact social group membership has on her life, whether she communicates with others to obtain information about their her group, and participates in cultural practices of her group. Ethnic affirmation subscale refers to a person's sense of group membership and attitudes toward the individual's group. The mean score for this subscale comes from seven questions within the full MEIM scale. It independently assesses clarity towards ethnic background, happiness with belonging, strength in belonging, amount she relates to, level of pride for, strength in attachment, and good feelings toward the ethnic group. The MEIM has shown comparable differentiation between various ethnic groups with an internal reliability of $\boldsymbol{\alpha}=.83$ for African Americans across all scales and $\boldsymbol{\alpha}=.87$ for its subscales (Brown et al., 2014). All 12 items that comprise the MEIM inclusive of the two subscales (ethnic search and ethnic affirmation) were used in the survey.

OGO acts as a mediating scale and is co-administered with the MEIM to explore a person's attitudes toward interacting with people from other ethnic groups. OGO scale mean score assesses a woman's attitude toward interacting with other ethnicities and is derived from six questions that individually ask whether she likes to meet and get to know other ethnicities, feels it is better that different ethnicities do not mix together, attempts friendships with members from other ethnic groups, is involved in activities with people from other ethnic groups, enjoys being around people from other ethnic 
groups, and amount of time spent with members of other ethnicities. The OGO has demonstrated less internal consistency in its use with alphas ranging from .35 (Ponterotto, Baluch, Greig \& Rivera, 1998) to .82 (Taub, 1995). Both MEIM and OGO use a 4-point Likert (strongly disagree to strongly agree) scale with the higher score indicating greater ethnic identity. A mean score is the preferred scoring method for the four scales (Yap et al, 2014). All six questions that make up the OGO were included in this survey.

Racial identity. Centrality subscale, one of four separate components of the Multidimensional Inventory of Black Identity (MIBI), was used to measure racial identity (Sellers et al., 1997). Eight questions in the centrality subscale collectively measure the extent race is a core component of an individual's self-concept. Individually the questions assess how being Black affects feelings about oneself, self-image, sense of what kind of person you are, reflections on who you are, plays a factor in one's social relationships, their sense of belonging to other Black people, their attachment to other Black people, and whether one's personal destiny is tied to other Black people. Centrality subscale also uses a 4-point Likert (strongly disagree to strongly agree) with the higher score indicating that race is a core component of self-concept. Strong internal reliability $(\boldsymbol{\alpha}=.79)$ was found for this measure with African-American and AfroCaribbean women (Sussner et al., 2010). All items created for the centrality subscale were included in this survey.

Social cohesion. Social cohesion was measured by three questions, "This question is about your general feeling of trust that you have for your neighbors." Following this lead-in statement the line of questioning continued with, "Would you say 
that most of the time people in your neighborhood try to be helpful, or are they mostly looking out for themselves," and "Have you volunteered or been a member of any of these groups within the past 3 years?" These items came from Kawachi and colleagues (1999) to measure social cohesion.

Community cohesion. Three questions were used to measure community cohesion with ordinal data. These questions came from 2005 Citizenship Survey, London, England, that was originally used to assess social cohesion (Laurence, 2011). They asked, "To what extent do you agree or disagree that this local area is a place where people from different backgrounds get on well together? How much do you agree or disagree that it is possible to fully belong as a US resident and maintain separate cultural or religious identity? What proportion of people in this local area are of the same ethnic group as you?" A 5-point Likert scale was used to answer the first two questions. A 4point Likert was used for the final question. Higher scores indicated more cohesion in the social networks. The items, "How much do you agree or disagree that it is possible to fully belong as a US resident and maintain separate cultural or religious identity," "How important is your ethnic or racial background to your sense of who you are," "How important is your national identity to your sense of who you are," and "How important is the country your family came from originally to your sense of who you are" were taken the items from the 2005 Citizenship Survey to explore community cohesion. Reliability information was not available on these items. All of these questions were used in this survey.

Health orientation and health-seeking. Thirteen items were used in this series. The first of 13 items, "How is your health in general?" was a 5-point Likert scale taken 
from NHANES 2011-2012 CAPI Questionnaire (CDC, 2012) ranging from Excellent to Poor. The remaining 12 items were developed for the cross-sectional survey. The 12 questions reflect the researcher's general interest in the women's access and use of health care services prior to participating in the research. They include, "Have you ever been tested for any of these diseases?" (Breast, Cervical, Colon) (yes/no for each disease), "Have you ever been told by a doctor that you have or have had any of these diseases?" (Breast, Cervical, Colon) (yes/no for each disease), "How often do you go to the doctor or another type of provider for health care?" (Never to Monthly), "How likely are you to go to a doctor or another provider just to stay healthy?" (Very unlikely to Very Likely), “How likely are you to go to a doctor or another provider to screen for cancer?" (Very Likely to Very Unlikely), "Do you have health insurance?” (Yes, No), “Do you plan to participate in the insurance exchange provided by the Affordable Care Act (Obamacare)?” (Yes, No), “Do you already have a primary care doctor?” (Yes, No), Where do you go if you need medical care?"(List of facilities), “Do you have your own form of transportation?" (Yes, No), "How far would you be willing to travel to see a doctor?_miles", "Are you working as an employee or are you self-employed?" (Employee, Self-employed, Unemployed). They have not been tested for internal reliability.

Perceived risks and screening beliefs. The Risk Behavior Diagnosis Scale (RBDS) (Witte et al., 1996) provides a 12-item scale that measured four distinct beliefs: disease severity, disease susceptibility, self-efficacy (for carrying out the behaviors), and response efficacy (whether the behavior will produce a positive result). The scale has been validated for condom use, seatbelt use, and colon cancer screening with Cronbach's 
alpha ranging from 0.71 to 0.90 . Dassow (2005) adapted this scale to measure women's beliefs about breast cancer, colon cancer, and osteoporosis. RBDS was used to measure perceived risk and beliefs about breast screening. A 5-point Likert (ranging from strongly disagree to strongly agree) were the responses; the higher score indicating stronger agreement with the statement made in the question item. All questions in the RBDS were included in this survey.

Screening status. The following two questions documented whether the women had screened for breast, cervical, or colon cancer in the past and the outcome of these exams. The first question asked, "Have you ever been tested for any of these diseases (breast cancer, cervical cancer, colon cancer)". The second question asked, "Has a doctor ever told you that you have (breast cancer, cervical cancer, colon cancer)". Both questions were used to determine pre-existing screening status at the time they completed the survey. Reliability information was not available for these questions.

Screening intention. The original question written to address behavioral intention was changed from "Do you plan to take a mammogram exam?" to "Do you plan to visit the Broward Department of Health, your doctor or a local clinic within the next 3 months to get your breast examined?" The language was changed to support the following questions on awareness of screening services in the community and to bind the question within a specific time frame. Yes, no and not sure were the possible response options in this series. These questions were written for this study and reliability information is not available.

Screening barriers. One question was originally created to address screening barriers, but this was changed after consultation with the primary advisor. Individuals 
were originally asked to rank from 1 to 15 the reasons that would prevent them from screening for breast cancer. Most of the response options originated from patient anecdotal data on a FIU colon cancer study (McKinney \& Palmer, 2010), barriers listed in the literature, and comments made by community members. The question was revised to a list of barriers. For each barrier, women were asked to provide two separate responses for each topic: indicate that she agreed that the listed topic made it (1) difficult to see a doctor and that topic (agreed/disagreed) and the topic (2) prevented her from going to the doctor (yes/no). The intent was to document the issues that made it difficult to screen and distinguish from this list those issues that prohibited screening. These questions were written for this study and reliability information is not available..

Awareness about Broward Department of Health screening resources. Three questions were used to determine the extent to which women were aware of Broward county sponsored screening resources. The questions were, "Did you know that Broward's Department of Health has a program to provide breast exams (mammograms) and PAP smears," "Have you ever used these services," and "Do you have friends, family, or neighbors who have used these services". Response options for these questions were yes, no and not sure. These questions were written by the researcher for this study.

Importance of identity to define sense of self. Three questions were used to create a single dichotomous variable for importance of identity to define sense of self. This was done was to develop a measure that offered commentary on the overall influence in identity in the respondent's everyday sense of who they are. The questions were Importance of racial and ethnic background to sense of self," "Importance of national identity to sense of self," and "Importance of home country to sense of self." 
The original scale was a 4-point Likert that ran from 1, "not at all important" to 4, "very important." The new response options were "low importance" and "high importance." This item was developed for this study and reliability information is not available.

\section{Content Validity of Survey Instruments}

English-language instrument. Content validity of the English language survey was obtained through a pilot test with a sample of ten English-speaking women who resided in the target community and met the inclusion criteria. Five of the panelists opted to use the electronic modality and five selected the paper survey. The women were timed and their comments regarding clarity, accuracy of the directions, readability, burden, and contextual meaning were recorded and used to revise the final document. Suggestions included that a separate series of items tailored to topic of identity was unnecessary and should be removed. Panel verified that the locations of possible medical care venues included in Q41 was appropriate and exhaustive for women in the community. Members also added Q51 to correctly account for women who may respond no to the preceding question.

Haitian Creole-language instrument. A systematic process of translation, verification, and testing was used to validate the data collection tools in Haitian-Creole. Three research assistants, fluent in Haitian-Creole, collectively translated the Englishlanguage survey, recruitment materials, and IRB-approved consent form to Creole language. Each document was divided into three sections and, in rotation, one person would translate a segment while the other two reviewed the content for accuracy to English-language documents, consistency, and cultural relevance. Consensus was used to select wording that held meaning across the various regional and class dialects of Creole. 
For instance, where medical or procedural terms did not exist in the Creole language, words from the French vocabulary were used along with a Creole-language explanation of the French term inserted alongside the French language word. Readability was then tested with a community-member sample of four Haitian women and their comments were incorporated into the next draft. The Haitian language survey was back translated into English by research staff. A seasoned Haitian medical researcher reviewed the draft for accuracy of medical terms and overall congruency with the English-language document. The document in both languages was sent to an approved translation vendor that certified the translations before use.

\section{Phase 3. Participation in Semi-Structured Discussion Groups}

Discussion group settings. Of 16 groups completed, 14 had clear audio. Four were included in this dissertation. Data collection occurred in two venues within the targeted neighborhood. The first was a centrally located public library. This library was patronized daily for educational, social, and community activities. Study participants visited during regular business hours with research activities occurring in a private, first floor seminar room in the building. Discussion groups were held at this site from October 2015 to February 2016. The second venue was a worship center that served a predominately Haitian congregation and provided social support services to the larger neighborhood. Here, the study was conducted within a community recreation hall adjacent to the faith center. One day was dedicated to data collection, one session was held in the afternoon and another in the evening.

Discussion group procedures. Each discussion group followed the same

process. The researcher (a) welcomed the women and provided an overview of the study, 
(b) confirmed their interest in continuing with the study, (c) offered the option of completing the consent and survey document using audio, written, or verbal communication, (d) provided ample time for participants to read and discuss the consent form, (e) had the participants sign the consent form that provided permission to tape record the session, (f) provided a signed copy of consent to the participants for their records, $(\mathrm{g})$ requested the participant to complete the cross-sectional survey instrument, and (h) conducted the discussion group. Each participant was given the option to end the session at any time without penalty.

Upon completion of the discussion group session, participants received a $\$ 30.00$ store gift card for completing both the survey and discussion group. A $\$ 15.00$ card was also given to the women who only completed the survey. Participants were also notified that the findings would be shared publicly at the African-American Research Library and Cultural Center upon completion of the write-up and doctoral defense.

Each discussion session was recorded using a digital audio taping device. Each digital file was saved on the researcher's computer and labeled by discussion topic (ethnic identity or cultural identity), the groups' ethnic composition (African American, Caribbean, Mixed Ethnicities), and date. The digital files were destroyed at the completion of the analysis process.

Fourteen group discussions using a semi-structured interview guide were used to collect data on personal meanings attributed to culture and ethnic identity and offer strategies for using these concepts to promote breast screening in ZIP-Code 33311. Eight of these groups addressed culture as their primary topic of interest and five groups addressed ethnic identity. One additional session was held to clarify prior comments 
made across all of the sessions. A third-party transcription company transcribed group discussion sessions. Transcribed documents were reviewed to verify accuracy. Thematic analysis was conducted by the researcher who used both pre-set and open codes to analyze four transcribed one-hour semi-structured group discussions. SPSS version IBM 10 was used to facilitate quantitative analyses.

\section{Semi-structured discussion group guide.}

Phase 3 was directed by a semi-structured discussion group guide (Appendix B). One guide was created to lead the semi-structured discussions and the same guide was tailored to support two thematically different discussions where one addressed the relationship of culture and health and the second on ethnic identity and health. This guide was designed to explore the meanings of culture and ethnicity, their relationship to women's perspectives about their health, and suggest ways that ethnicity and culture could be used to improve Black women's involvement in mammograms and preventive medical visits.

The discussion guides were developed over the course of four months and each consisted of 19 questions. Prior experiences as a volunteer for five years with a local breast cancer support and educational program for women of color and as a volunteer on a recent project to conduct focus groups in Dade and Broward county for a local chapter of Susan G. Komen regional office provided insight into the special challenges that Black women were facing in their journey with breast cancer. In particular, these experiences informed the researcher that culture influenced their comfort to talk about breast cancer and to seek help with this disease. Likewise, for many Black women their sense of support and empowerment was linked to their bonds with other Black women. To the 
extent that these resonated for more Black women in Broward was unknown. The questions in the guides were based upon the experiences and perspectives encountered in these volunteer activities.

Faculty members of the dissertation committee were consulted in the development of the two discussion guides. They provided direction regarding the construction of the preliminary constructs, sequencing of the questions, and scope of the questions. A convenience sample of three adults reviewed the questions for readability and comprehension. Changes were made to the instrument based upon their comments. A second convenience sample comprised of members from the community reviewed the questions for readability, relevance, and comprehension. Two guides were created from the review. Both guides contained 19 questions that were arranged in four major sections: (1) participants' meaning of culture or ethnic identity, (2) relationship of ethnic identity or culture to health seeking behaviors of themselves and other Black women, (3) their personal experiences with mammograms, and (4) additional comments.

The discussion guides were developed to contextualize the meanings of the terms ethnicity and culture to the lived experiences of women of Haitian, Caribbean, and African-American descent who lived in the community. The researcher's theory was that the meaning of these terms would be unique to the community of women based upon a presumed diversity in their social norms, social networks, and lived experiences. Likewise, these nuances would differ by their ethnic membership. The guides were created specifically to articulate the meanings of ethnicity and culture from the perspective of Black women who lived in the target area; to document their rationale for Black women in the community not being more engaged with preventive health practices, 
particularly in a comprehensive breast health plan; articulate their suggested strategies for using ethnic identity and cultural identity to better promote breast health; and to document their lived experiences with mammography and breast cancer.

English Language Guides. Questions were reviewed by two faculty members who suggested edits to the questions that were selected for inclusion, to the wording of the questions, and to the sequencing of the questions in the guides. The guides were then piloted with a group of three women who provided comment on clarity, comprehension and cultural appropriateness of the questions. The completed guide was then translated in Haitian-Creole language. The translation process was conducted in the same manner described for the cross-sectional survey and completed by the same research assistants. As with the cross-sectional survey, the guide was reviewed by an external language specialist, reviewed by Haitian women who were fluent in Creole, and edited by an FIU researcher also fluent in Haitian-Creole. After each review, revisions were made to address clarity, wording, and accuracy in response to formative recommendations made by community members.

The semi-structured discussion group guide was used to collect data for three domains: the meanings of culture and ethnic identity, the influence of culture on health seeking behaviors or the meaning of ethnicity on health seeking behaviors, and women's perspectives about mammograms. These questions were developed in consultation with the major professor and committee members with expertise in qualitative research methods. 


\section{Data Analysis}

This section describes how both the survey data and discussion group data were processed and analyzed for this study. This description was divided by the typology of the data (survey or discussion group). The specifics were ordered with a presentation first of the quantitative then qualitative analyses.

\section{Quantitative Analyses}

\section{Research Question 1}

To test the first hypothesis two separate analyses addressed health perceptions and four others explored health-seeking behaviors. Mann-Whitney U nonparametric test of independent samples was used to compare the distribution of RBDS measures for cancerrelated perceptions and attitudes about breast self-exam between African American and Caribbean women. Univariate and multivariate regression was used on RBDS measures for cancer-related perceptions and attitudes regressed on ethnic identity controlling for covariates.

Bivariate analyses were conducted to describe self-reported behaviors of AfricanAmerican and Afro-Caribbean women regarding those who had ever completed a test for breast, cervical, and colon cancer, as well as, their use of a primary care doctor and nonWestern medical providers. Crosstabs were conducted to explore the ethnic distribution of women who were likely to see a doctor as a preventive medical visit and women who were likely to see a doctor to screen for cancer. Finally, crosstabs explored the association between awareness of Broward County Department of Health services and use of screening services. Crosstabs were created to explore this relationship for the 
overall sample of women and compared outcomes of African-American with AfroCaribbean women.

\section{Research Question 2.}

The second hypothesis used binary logistic regression to develop regression models for three separate health-seeking behaviors: a) complete a mammogram, b) seeing a doctor before being sick and c) seeing a doctor to test for cancer. In model 1 complete a mammogram was regressed on overall attitude about cancer controlling for covariates. In model 2 seeing a doctor before being sick was regressed on ethnic identity controlling for covariates. Similarly, in model 3 seeing a doctor to test for cancer was regressed on ethnic identity controlling for covariates.

\section{Research Question 3.}

The third hypothesis used binary logistic regression and social cohesion was regressed on ethnic identity represented by ethnic membership and MEIM total score controlling for covariates.

All analyses were conducted using SPSS version from IBM SPSS Statistics 21. Because not all participants had complete data for each variable, missing data values were imputed using packaged function of IBM SPSS Statistics 21.

\section{Qualitative Analyses}

Assembled teams to assist with qualitative analyses. A thematic analysis of four transcripts was conducted by two, two-person teams (Team A and Team B). The four persons were ethnically diverse, representing the ethnicities of the study participants, familiar with the target community and trained in qualitative analysis. The selected 
transcripts were discussions that had the fewest missing items from the designated interview guide. Two transcripts were assigned to Team A and two to Team B.

The teams did not complete the entire analysis of the qualitative data as planned but had offered input into the development of the initial set of open codes. The external reviewers did not complete this process because of conflicting personal and work-related obligations, loss of interest in the project, and unavailable time to perform this work. This researcher then built upon the initial codes that were identified by the team and independently consolidated this list so that is was responsive to the goals of this project and preceded to develop the appropriate themes and categories included in this dissertation. The remainder discussion is written from the perspective of the post-team, analyses.

Steps taken by reviewers in the qualitative analyses. The coding and analysis of the four discussions were guided by a combined methodology that used pre-set codes and “open coding." The preset codes were doctor's visit, screening, mammogram, culture, and prevention. The parameters of the open coding were any issues, people, or perspectives that were related to seeing a doctor for a preventive care visit and seeing a doctor to test for cancer.

The methods required three independent reads of each transcript. The transcripts were reviewed first for context and familiarity of the conversations. The second review was for the purpose of identifying any words or phrases that were related to the two psychosocial constructs (culture and social cohesion) and the two health-seeking behaviors of interest in this study (action as a preventive health care visit and action to screen for cancer). A distinction was then made for items that appeared in all four 
discussions or only in a discussion among primarily African American or Haitian participants. This researcher conducted a final review using a process referred to as "open-coding" to identify other pertinent themes not captured by the pre-set codes to document the women's operational definition for the term culture.

A systematic process was adopted to guide the decision making in this analysis. First, the reviewer studied the transcript to identify passages that suggested relevance to the pre-set codes. Each selected passage was transferred electronically to the thematic matrix and similar categories were grouped together (Appendix C). Categories were created for each area of interest in this study to suggest a structure for the open-codes that transcended across all four interviews and identified within-group differences by ethnicity (African American versus Haitian). Themes associated with the broader research interests of culture, social cohesion, health perceptions, and breast exams that were identified by the participants were reported on the matrices. This researcher further reduced the content of the themes related to the broader research interests of this study and reported this information in this dissertation by the self-described ethnic membership of the participants.

Steps taken by researcher to prepare data for the coding process. Additional steps were conducted to support this analysis. A third-party, transcriptionist created transcripts for 11 of the 15 interview sessions. The researcher transcribed three of the remaining interviews resulting in 14 of the 15 interviews transcribed. Audio was not properly recorded for the $15^{\text {th }}$ sessions. This researcher also reviewed each audio with the written notes made during the sessions to familiarize with data. 
Transcripts were separated by topic area (cultural identity or ethnic identity). This dissertation reports a subset of the data collected during this process. Four cultural identity transcripts were selected for analysis because they (a) followed closest to the interview guide and (b) the guide was administered to the each of the available ethnic demographics for this study (African American, Caribbean, Haitian in English and Haitian in Creole). The content of each transcript was then entered into an MS Excel spreadsheet that had been created to support the data analysis. The spreadsheet included 16 tabs. Each tab was dedicated to the individual questions included in the discussion guide. .

Coding considerations. This researcher read the transcribed content that had been entered into the MS Excel spreadsheets and coded the information to identify responses that were relevant to the aims of this study. This process required that the researcher identify content that represented components of the underlying concepts related to the constructs cultural identity and culture.

For cultural identity, the researcher selected excerpts of each discussion that identified the persons, extended family, and community norms that influenced their health-seeking behaviors-including the uptake of mammograms and completion of a preventive medical visit. Excerpts were selected that explained the women's perceptions about receiving mammograms, screening for cancer, and completing a preventive health care visit- describing the factors that enabled or hindered their participation in these behaviors. This researcher also noted from the transcripts evidence of those aspects found in the women's culture that had a positive or negative influence on their health behaviors and areas where the participants suggested that their culture could be used to support 
women to adhere more fully with breast screening recommendations. Coding considerations, especially those related to culture, were influenced by the methodology and rationale of Airhihenbuwa (1995).

The final step of the analysis process involved developing a summation of the comments made by the participants in the discussions. The researcher assigned the codes by the characteristics of the group and to the factors that frame each of the discussions. Specifically, the ideas offered in the discussions identified factors that translated to the beliefs and perspectives within the community and among Black women that influenced participation in mammograms. The suggestions were stratified along nativity and ethnicity to identify comments that were common or varied by these demographics whenever possible.

\section{Dissemination Strategies}

Several actions have been planned to disseminate the findings from this dissertation to key individuals and organizations that are responsible for health policy and programming in Broward County. Findings from this study will be disseminated in a community forum within 60 days following the defense. In addition to the public forum, one paper is currently being developed by the researcher and the major faculty advisor for publication. Findings from this study have also translated to support the development of a breast health intervention that empowers Black women to transition towards a comprehensive approach for breast health care entitled BCM model: breast self-exam (BSE), clinical breast exam, and mammograms. 


\section{Summary}

Chapter 3 detailed the analytical methods used in this mixed-methods study.

Three hypotheses were proposed in the quantitative component of this study.

Independent samples $t$ test was conducted for the first analysis and binary logistic regression for the remaining two hypotheses. Qualitative methods were used to code the transcriptions from four semi-structured interviews to potential influence culture, social cohesion, and women's health perceptions to women seeking preventive medical health care, completing a mammogram, and screening for cancer. Pre-set and open-coding were used to analyze these discussions. Chapter 4 details the findings from these analyses.

\section{LIST OF REFERENCES}

Airhihenbuwa, C. O. (1995). Health and Culture Beyond the Western Paradigm. Thousand Oaks, CA:Sage Publications.

Bowen D, Hickman KM, Powers D. (1997). Importance of psychological variables in understanding risk perceptions and breast cancer screening of African American women. Women's Health, 3(3-4):227-42.

Braun, V \& Clarke, V. (2006). Using Thematic Analysis in Psychology. Qualitative Research in Psychology, 3(2), 77-101. doi:10.11912/1478088706qp063oa

CDC (2012) NHANES Computer Assisted Personal Interview. Retrieved December 13, 2013 http://www.cdc.gov/nchs/nhanes/nhanes2011-2012/questexam11 12.htm

Dassow, P. (2005). Setting educational priorities for women's preventive health: measuring beliefs about screening across disease states. Journal of Women's Health, 14(4), 324-330. doi: 10.1089/jwh.2005.14.324

Florida Department of Health (2015). Annual Legislative Report of the Florida Breast Cancer Early Detection and Treatment Referral Program. Retrieved from http://www.floridahealth.gov/diseases-and-conditions/cancer/breastcancer/ documents/bcc-2013-legislative-report.pdf

IBM SPSS Statistics 21 
Kawachi, I., Kennedy, B.P. \& Glass, R. (1999). Social Capital and self-rated health: A contextual analysis. American Journal Public Health, 89(8), 1187-1193.

Kobetz, E. \& Parker, D. Cancer Data for South Florida: A Tool for Identifying Communities in Need, Report to the Health Foundation of South Florida. Disparities and Community Outreach Core, University of Miami Sylvester Comprehensive Cancer Center; 2010. Research Funded by Health Foundation of South Florida and community-based organizations.

Laurence, J. (2011). The effect of ethnic diversity and community disadvantage on social cohesion: A multi-level analysis of social capital and interethnic relations in UK communities. European Sociological Review, 27(1), 70-89.

Levy, A. R., Bruen, B. K., \& Ku, L. (2012). Health Care Reform and Women's Insurance Coverage for Breast and Cervical Cancer Screening. Preventing Chronic Disease, 9, E159. http://doi.org/10.5888/pcd9.120069

McKinney S, Palmer R. (2013). The Influence of Gender on Colorectal Cancer Knowledge, Screening Intention, Perceived Risk and Worry among African Americans in South Florida. Journal of Community Health, 39, 230-238. doi: $10.1007 / \mathrm{s} 10900-013-9812-8$

Plescia, M., Wong, F. L., Pieters, J., \& Joseph, D. (2014). The National Breast and Cervical Cancer Early Detection Program in the Era of Health Reform: A Vision Forward. Cancer, 120(0 16), 2620-2624. http://doi.org/10.1002/cncr.28826

Ponterotto, J.G., Galuch, S., Greig, T., \& Rivera, L. (1998). Development and initial score validation of the Teacher Multicultural Attitude Survey. Educational and Psychology Measurement, 58, 1002-1016.

Sellers, R.M., Rowley, S.A., Chavous, T.M., Shelton, J.N. \& Smith, M.A. (1997). Multidimensional Inventory of Black Identity: a preliminary investigation of reliability and construct validity. Journal of Personal Social Psychology, 73, 805815.

Sussner, W.M., Edwards, T.A., Thompson, H.S., et al. (2010). Ethnic, racial and cultural identity and perceived benefits and barriers related to genetic testing for breast cancer among at-risk women of African descent in New York City. Public Health Genomics, 14, 356-370.

Urban League of Broward County, (2013).Closing the Gap. The State of Black Broward: Health Report. Retreived from ULbroaward.org August 8, 2016.

US Census, 2014a. 2010-2014 American Community Survey 5-Year Estimates. Selected characteristics of the native and foreign born populations for Broward County. U.S. 
Census Bureau, 2010-2014 American Community Survey 5-Year Estimates.

Retrieved from

http://factfinder.census.gov/faces/tableservices/jsf/pages/productview.xhtml? src=C $\underline{\mathrm{F}}$

US Census, 2014b. 2010-2014 American Community Survey 5-Year Estimates. Selected characteristics of the native and foreign born populations for 33311. U.S. Census Bureau, 2010-2014 American Community Survey 5-Year Estimates. Retrieved from

http://factfinder.census.gov/faces/tableservices/jsf/pages/productview.xhtml? src=C $\underline{\mathrm{F}}$

Witte K, Cameron KA, McKeon AJK, Berkowitz JM. (2012) Predicting Risk Behaviors: Development and Validation of a Diagnostic Scale. Journal of Health

Communication, 1, 317-341.

Yap, S. C. Y, Schwartz, S. J., Kim S. Y., .., \& Vazsonyi, A. T. (2014). /investigating the Structure and Measurement Invariance of the Multigroup Ethnic Identity Measure in a Multiethnic Sample of College Students. Journal of Counseling Psychology, 61(3), 437-446. doi: 10.1037/a0036253 


\section{CHAPTER IV: RESULTS}

Quantitative methods were selected to collect data during Phase 2. The findings from Phase 2 describe the distribution of health behaviors among women and examine the influences of ethnic identity and social cohesion on health-seeking behaviors. Qualitative methods were selected to collect data during Phase 3. The findings from Phase 3 identify the social determinants, cultural and community norms and class and income factors that influence delay or avoidance of mammography for African-American and Afro-Caribbean women.

\section{Presentation of Phase 2 Data}

Section 1 describes the sample's demographics and Section 2 presents the quantitative findings.

\section{Section I: Description of Study Participants}

A total of 121 women were enrolled into this study and 117 were included in the analyses because five or more responses in the MEIM scales were missing for four participants. Over one half (51.3\%) of the women self-described as Afro-Caribbean and the remainder as African-American (48.7\%). Age groups were evenly distributed with $31.6 \%$ being $40-49$ years old, $31.6 \%$ being $50-59$ years old, and $36.8 \% 60$ years and older. Most of the women who participated in this study had been long-time residents with $84.6 \%$ having lived in Broward at least 10 years and $70.1 \%$ having lived in the predominately Black population areas at least 10 years. The sample was equally represented by women who lived in the ZIP-code $33311(51.3 \%)$ and those who lived in the ZIP-codes adjacent to ZIP-code 33311 (48.7\%). Most had at least a high-school education (64.1\%), most had some form of health insurance $(76.9 \%)$, and most indicated 
that they had a primary-care physician (83.8\%). Other characteristics that may have moderated health behaviors included: almost one-fifth of the sample had sought medical services from a non-westernized health provider, most considered their health to be poor (73.5\%), and most were foreign-born (56.4\%).

\begin{tabular}{|c|c|c|c|}
\hline & $\begin{array}{l}\text { Caribbean / } \\
\text { West Indian }\end{array}$ & $\begin{array}{c}\text { African } \\
\text { American } \\
\end{array}$ & \\
\hline Covariates & n (\%) & n (\%) & $X^{2} P$ \\
\hline Age & & & .30 \\
\hline $40-49 * *$ & $21(35.0)$ & $16(28.1)$ & \\
\hline $50-59$ & $21(35.0)$ & $16(28.1)$ & \\
\hline $60+$ & $18(30.0)$ & $25(43.9)$ & \\
\hline Self-identified race & & & .01 \\
\hline Black & $53(88.3)$ & $57(100.0)$ & \\
\hline Other & $7(11.7)$ & $0(0.0)$ & \\
\hline Educational Attainment & & & .86 \\
\hline Less than High (primary) School** & $22(36.7)$ & $20(35.1)$ & \\
\hline High (primary) School and Above & $38(63.3)$ & $37(64.9)$ & \\
\hline Income & & & .01 \\
\hline$\$ 15,000$ and Above & $20(33.3)$ & $34(59.6)$ & \\
\hline Under $\$ 15,000^{* *}$ & $40(66.7)$ & $23(40.4)$ & \\
\hline Residency & & & $<.001$ \\
\hline Resident of ZIP-code $33311^{* *}$ & $20(33.3)$ & $40(70.2)$ & \\
\hline Resident in Adjacent ZIP-codes & $40(66.7)$ & $17(29.8)$ & \\
\hline Length of Time In Target & & & .10 \\
\hline Less than 10 Years** & $22(36.7)$ & $13(22.8)$ & \\
\hline $10+$ Years & $38(63.3)$ & $44(77.2)$ & \\
\hline Length of Time In County & & & .16 \\
\hline Less than 10 Years** & $12(20.0)$ & $6(10.5)$ & \\
\hline $10+$ Years & $48(80.0)$ & $51(89.5)$ & \\
\hline Foreign-Born Status Participant & & & $<.001$ \\
\hline Participant and Parents US-Born & $7(11.7)$ & $56(98.2)$ & \\
\hline Either Parent or Participant & $3(5.0)$ & $1(1.8)$ & \\
\hline $\begin{array}{r}\text { Both Participant and Parents } \\
\text { Foreign-Born** }\end{array}$ & $50(83.3)$ & $0(0.0)$ & \\
\hline $\begin{array}{r}\text { Importance of Identity to Sense } \\
\text { of Self }\end{array}$ & & & .30 \\
\hline High Importance & $4(6.8)$ & $7(12.5)$ & \\
\hline Low Importance & $55(93.2)$ & $49(87.5)$ & \\
\hline
\end{tabular}


Bivariate analyses were performed to compare the distribution of AfricanAmerican and Afro-Caribbean women across nine socio-demographic characteristics. Four characteristics showed a statistically significant difference $(\mathrm{p}<0.05)$ between African-American and Afro-Caribbean women (Table 1).

All African-American women self-identified their race as Black while a significant number of Afro-Caribbean women did not self-describe their race as Black $(12.5 \%, \mathrm{p}<0.01)$. African-American women were more likely to report their annual salary as above $\$ 15,000(59.6 \%)$ compared to Afro-Caribbean women $(36.5 \%, \mathrm{p}<0.01)$. Afro-Caribbean women lived both within (33.3\%) and outside (66.7\%) Zip-code 33311 compared to African-American women who were more likely to live within ZIP-code 33311 (70.2\% versus $29.8 \%$ ). Afro-Caribbean women were also more likely to be foreign-born than African-American women $(\mathrm{p}<0.01)$.

Two of the seven variables that measured community norms showed statistically significant differences by ethnicity. Afro-Caribbean women were more likely to use a non-western medical provider $(\mathrm{p}<0.03)$ and less likely to have previously screened for colon cancer $(\mathrm{p}<0.05)$. Additional community norms investigated for statistically significant differences by ethnic membership included having a primary care doctor, insurance status, perceived health, screened for cervical cancer, and awareness that DOH provides breast screening services in the community. None of these were found to be significant at the $95 \%$ level. Most of the women had primary care providers, although more Caribbean women were without one (23.8\%) compared to African-American women (10.5\%). While most women had health insurance (76.9\%), more Afro-Caribbean women were without (28.3\%) compared to African-American women (17.5\%). Finally, 
almost one-third of both African-American and Afro-Caribbean women perceived their health as poor.

Ethnic identity had been conceptualized as consisting of two independent measures. The first was ethnic group membership, a nominal variable created to communicate the self-described ethnicities of the women as either Afro-Caribbean of African American. The second was the presentation of the MEIM and the centrality scale. The MEIM included three scales and a companion scale, the other group orientation scale (OGO), and a centrality scale. Collectively, they indicated the values that a person had attached to their ethnic origin and signaled its influence in the conduct of everyday activities, creation of interpersonal relationships, and decision making. African-American and Afro-Caribbean women were different with respect to the OGO $(\mathrm{p}<0.01)$ suggesting that African-American women were more receptive and engaged with women who were not of the same ethnicity in contrast to Afro-Caribbean women in Broward County.

Social cohesion was an independent variable that addressed community norms. Social cohesion represented communication and interaction among people to share information, protect and promote community interests, and included social networks within the relationships of the community. African-American women (66.7\%) were found to have more socially cohesive characteristics than Afro-Caribbean women (43\%, $p<.01)$ (Table 2).

Importance of identity to self, referred to the level of importance one's identity had on decision making, social activities, priorities, and behavior. Bivariate analyses by ethnicity showed that both African-American (87.5\%) and Afro-Caribbean women (93.2\%), indicated that this construct was very important to their self-concept $(p=0.30)$. 
This construct was influential as an independent variable for measuring community norms. Most women had screened at least once for colon cancer $(p=0.05)$, almost half of the women had not been examined for cervical cancer $(\mathrm{p}=.0 .78)$, and most perceived their health to be poor $(\mathrm{p}=0.88)$.

\begin{tabular}{|c|c|c|c|c|}
\hline Outcome Variables & $\begin{array}{l}\text { Caribbean / } \\
\text { West Indian }\end{array}$ & $\begin{array}{c}\text { African } \\
\text { American }\end{array}$ & & \\
\hline & n (\%) & n (\%) & $\mathbf{n} / \mathbf{a}$ & $P$ \\
\hline \multicolumn{5}{|l|}{ Health-Seeking Behaviors } \\
\hline Completed a Mammogram & & & & .08 \\
\hline Yes & $45(75.0)$ & $50(87.7)$ & & \\
\hline No & $15(25.0)$ & $7(12.3)$ & & \\
\hline See Doctor for Preventive Exam & & & & .08 \\
\hline Yes & $43(72.9)$ & $49(86.0)$ & & \\
\hline No & $16(27.1)$ & $8(14.0)$ & & \\
\hline See Doctor to Screen for Cancer & & & & .10 \\
\hline Yes & $43(72.9)$ & $47(85.5)$ & & .09 \\
\hline No & $16(27.1)$ & $8(14.5)$ & & \\
\hline \multicolumn{5}{|l|}{ Community Norm } \\
\hline Measure of Social Cohesion & & & & .01 \\
\hline Low/Weak & $34(56.7)$ & $19(33.3)$ & & \\
\hline High/Strong & $26(43.3)$ & $38(66.7)$ & & \\
\hline Perceptions & Mean (SD) & Mean (SD) & CI & $\boldsymbol{P}$ \\
\hline $\begin{array}{r}\text { Overall Attitude about the } \\
\text { Disease }\end{array}$ & $3.81(.65)$ & $3.91(.45)$ & $\begin{array}{c}(-.100 \\
.309)\end{array}$ & .31 \\
\hline Perceived Urgency & $4.49(.94)$ & $4.61(.77)$ & $\begin{array}{l}(-.197 \\
.429)\end{array}$ & .46 \\
\hline Benefit to Screening & $3.89(1.08)$ & $4.00(.81)$ & $\begin{array}{c}(-.236 \\
.458)\end{array}$ & .53 \\
\hline Perceived Risk & $2.78(1.25)$ & $2.82(.91)$ & $\begin{array}{c}(-3.63 \\
.434)\end{array}$ & .86 \\
\hline Ability to do Self-Exam & $.406(.91)$ & $4.22(.74)$ & $\begin{array}{c}(-1.44, \\
.461)\end{array}$ & .30 \\
\hline
\end{tabular}




\section{Section II: Presentation of Quantitative Findings}

Q1. What were the perceptions and health-seeking behaviors about cancer among ethnically diverse Black women (African American and Afro Caribbean)?

\section{Exploring ethnic differences in health perceptions.}

$H_{0}$ : African-American and Afro-Caribbean women do not have a statistically significant difference in their perceptions and health seeking behaviors about cancer.

Risk Behavior Diagnosis Scales (RBDS). A Mann-Whitney U test was conducted to evaluate whether Afro-Caribbean women would score in the same manner, on average, in their overall health perceptions about (a) breast cancer, (b) sense of urgency about breast cancer, (c) risk to develop breast cancer, (d) ability to perform a breast self-exam, and (e) personal health benefit by completing a mammogram. Sense of urgency was not expected to rank higher for Afro-Caribbean than African American women (60.05 versus 57.89 respectively); however, this difference was not significant at the $95 \%$ level of confidence. None of the rank tests were statistically significant; therefore, the conclusion would be to fail to reject the null-hypothesis of no difference (Table 3).

\begin{tabular}{|c|c|c|c|c|}
\hline \multicolumn{5}{|c|}{ Table 6. Mean Rank of RBDS Scales by Ethnicity of Participants $(\mathrm{N}=117)$} \\
\hline & \multicolumn{2}{|c|}{ Mean Rank } & \multirow[b]{2}{*}{ z-score } & \multirow[b]{2}{*}{$p(\mathrm{x} \leq \mathbf{0 . 0 5})$} \\
\hline $\begin{array}{c}\text { RBDS Scale (perceptions and } \\
\text { attitudes) }\end{array}$ & $\begin{array}{c}\text { African } \\
\text { American }\end{array}$ & $\begin{array}{c}\text { Afro } \\
\text { Caribbean }\end{array}$ & & \\
\hline $\begin{array}{r}\text { Overall Attitude about } \\
\text { Disease }\end{array}$ & 61.45 & 56.68 & -.762 & .45 \\
\hline Urgency & 57.89 & 60.05 & -.413 & .68 \\
\hline Risk & 61.20 & 56.91 & -.697 & .49 \\
\hline Ability to do Self-Exam & 61.39 & 56.73 & -.758 & .45 \\
\hline Benefits & 60.12 & 57.93 & -.353 & .72 \\
\hline
\end{tabular}




\section{Exploring ethnic differences in health-seeking behaviors.}

$H_{1 B}:$ African-American and Afro-Caribbean women do not have a statistically

significant difference in their health-seeking behaviors about breast cancer and

other cancers.

Five health-related characteristics were referenced in this hypothesis: (a) number of women that had ever been screened for breast cancer, (b) number of women that had ever been screened for cervical cancer, and (c) number of women that had ever been screened for colon cancer, (4) number of women that had a primary care physician (PCP) at the time of completing the survey, and (5) number of women who used a nontraditional medical provider for their healthcare at the time of completing the survey. Bivariate crosstabs compared these five characteristics for African-American and AfroCaribbean women. Statistically significant differences were found between AfricanAmerican and Afro-Caribbean women who used a non-traditional healthcare provider $(p=.03)$ and women who had ever been screened for colon cancer $(p=.05)$. Although African-American women were more likely to indicate an intention for the remainder behaviors, these differences were not statistically significant at the $95 \%$ level of confidence (Figure 7). 


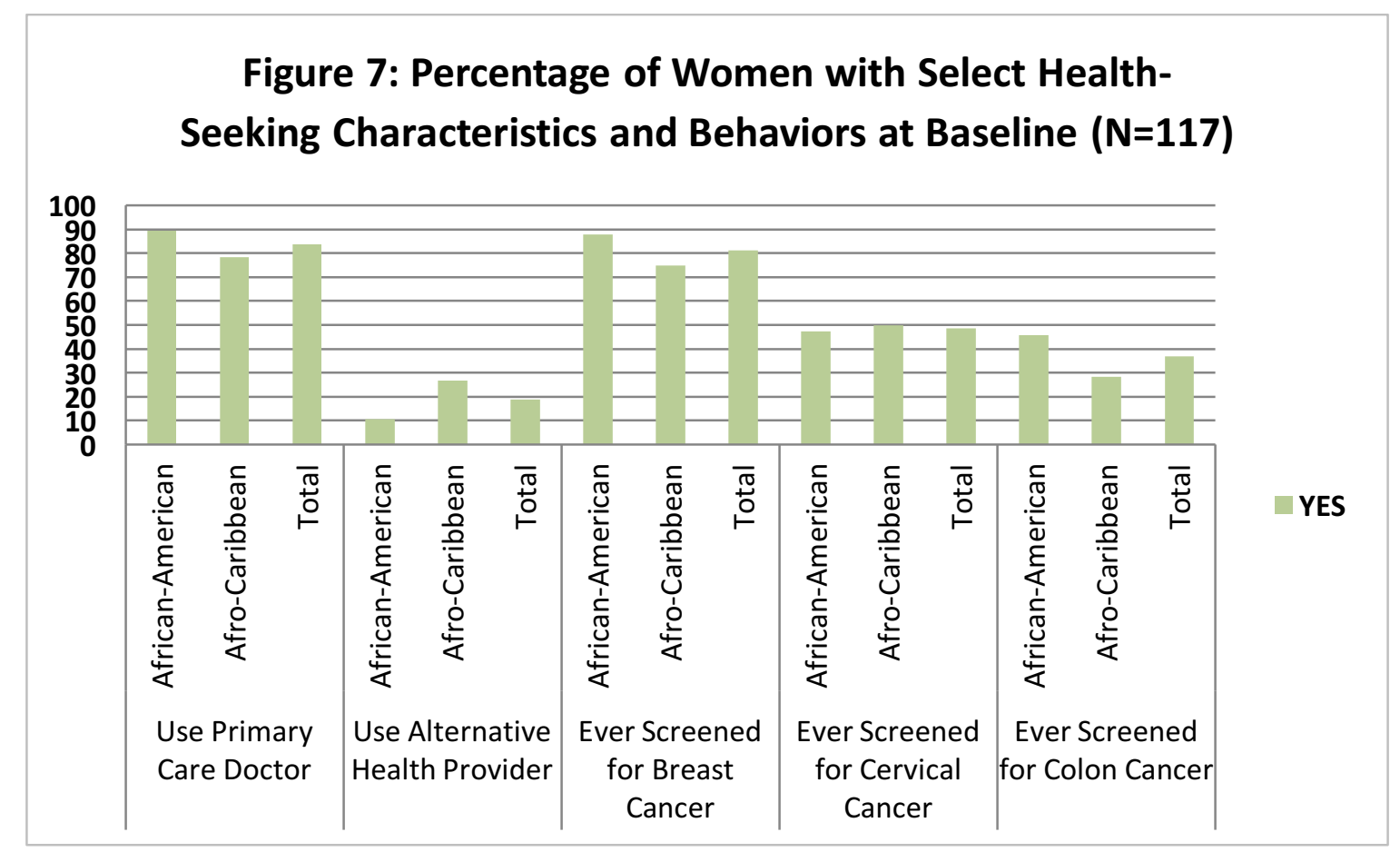

Exploring ethnic differences in health-seeking behaviors. Crosstabs examined differences between African-American and Afro-Caribbean women who expressed their intent to complete two separate health-seeking behaviors: a) the likelihood to go to a doctor or another professional before being sick, and 2) the likelihood to go to a doctor or other provider to screen for cancer.

Preventive medical visit. More women regardless of ethnicity indicated that they would participate in a preventive medical visit in the future. Almost twice as many AfroCaribbean women indicated that they would not participate in this activity capered to African-American. The difference between African-American and Afro-Caribbean women reporting they would not engage in preventive medical visits was not statistically significant at the $95 \%$ level of confidence level of $95 \%$. Therefore, fail to reject the nullhypothesis of no difference. (Table 7) 


\begin{tabular}{|c|c|c|c|}
\hline & Afro-Caribbean n (\%) & \begin{tabular}{|l} 
African American n \\
$(\%)$
\end{tabular} & p-value \\
\hline Unlikely & $16(27.1 \%)$ & $8(14.0 \%)$ & \multirow[t]{3}{*}{$\mathrm{P}=0.08$} \\
\hline Likely & $43(72.9 \%)$ & $49(86.0 \%)$ & \\
\hline Totals & $59(50.9 \%)$ & $55(48.2 \%)$ & \\
\hline
\end{tabular}

Medical visit to screen for cancer. More women, regardless of ethnicity, expressed a positive likelihood to see a doctor to screen for cancer. As with a preventive medical visit, a larger proportion of the African-American women would complete this exam than African-Caribbean women. Interestingly, comparing the two activities of African-American women were less likely to screen for cancer (85.5) compared to their involvement in a preventive medical visit (86.0\%). (Table 8). The difference between African-American and Afro-Caribbean women who reported they would screen for cancer was not statistically significant at the $95 \%$ level of confidence. Therefore, fail to reject the null-hypothesis of no difference.

\begin{tabular}{|r|c|c|c|}
\hline \multicolumn{1}{|c|}{ Table 8: Ethnic Differences in Likelihood of Women to Screen for Cancer (N=11) } \\
\hline & Afro-Caribbean n (\%) & $\begin{array}{l}\text { African American n } \\
(\%)\end{array}$ & \multirow{2}{*}{ p-value } \\
\hline Unlikely & $16(27.1 \%)$ & $8(14.5 \%)$ & \multirow{2}{*}{$\mathrm{P}=0.10$} \\
\hline Likely & $43(72.9 \%)$ & $47(85.5 \%)$ & \\
\hline Totals & $59(51.8 \%)$ & $55(48.2 \%)$ & \\
\hline
\end{tabular}

Multigroup Ethnic Identity Measure (MEIM). Univariate and multivariate linear regression analyses were performed to identify sociodemographic and ethnic factors that may have influenced the attitudes and perceptions of African-American and AfroCaribbean women regarding breast cancer and screening for cancer. Ethnic identity was documented by the MEIM and the scores for Black women who lived in Broward were 
compared to the scores of a similarly ethnically diverse sample of Black women who lived in New York City.

Women who lived in Broward had the following characteristics: more likely to have had a generally positive outlook about their ethnicity (Total MEIM), more involved in finding out information about their ethnic group (ethnic search), demonstrated that race has held a prominent role in a woman's self-concept (racial identity), and had maintained a greater sense of pride in being a member of their ethnic group (ethnic affirmation) than the women in the New York study. The women who participated in Broward appeared to be less socially involved with women outside of their ethnic groups than the women in the Sussner study. In comparison, four of the fives scales used to measure ethnic identity in Broward had mean scores that were 3.0 or higher (Table 9).

\begin{tabular}{|c|c|c|c|c|}
\hline \multicolumn{2}{|c|}{ Table 9. Mean Distribution of the Predicator Variable MEIM for Sussner (2010) and this } \\
Dissertation
\end{tabular}

Ethnicity in terms of ethnic group membership (Caribbean) was significantly associated with three of the five measures of attitudes and behaviors toward breast cancer 
and mammograms: overall attitudes $(p=0.05)$, sense of urgency $(p=0.04)$ and perceived risk $(p=0.02)$ (Table 10). A woman's score on the OGO scale was the only component of the MEIM ethnic identity measures that had a significant association. OGO scores were positively associated with overall attitudes toward breast cancer screening and breast cancer $(p=0.01)$, sense of urgency to screen $(p=0.03)$, perceived risk for developing the disease $(p=0.03)$, and perceived benefit of the test $(p=0.05)$ (Table 10$)$. OGO was not a significant predictor for perceived capacity to complete a self-exam, but educational attainment (less than high school) $(\mathrm{p}=0.04)$ and age $(40-49$ years $)(p=0.05)$ had statistically significant relationships with this behavior.

\begin{tabular}{|c|c|c|c|c|c|}
\hline \multicolumn{6}{|c|}{ Table 10: Factors that Influenced Women's Perceptions and Attitudes $(\mathrm{N}=117$} \\
\hline $\begin{array}{l}\text { Attitudes to } \\
\text { Influence }\end{array}$ & $\begin{array}{l}\text { Factors Found } \\
\text { to Influence } \\
\text { Behaviors } \\
\end{array}$ & $\begin{array}{l}\text { B coefficient/ } \\
\text { parameter } \\
\text { estimate }(\mathrm{SE})\end{array}$ & p-value* & $\begin{array}{l}\text { Confidence } \\
\text { Interval }\end{array}$ & $\mathbf{R}^{2}$ \\
\hline $\begin{array}{l}\text { Overall } \\
\text { Attitudes }\end{array}$ & $\begin{array}{l}\text { Ethnicity } \\
\text { Other Group } \\
\text { Orientation } \\
\text { (OGO) } \\
\text { Age 40-49 } \\
\text { Age 50-59 }\end{array}$ & $\begin{array}{l}-.440(.23) \\
.324(.12) \\
\\
-.490(.14) \\
-.270(.14)\end{array}$ & $\begin{array}{l}0.05 \\
0.01\end{array}$ & $\begin{array}{l}(-.889, .009) \\
(.092, .555) \\
\\
(-.772,-.209) \\
(-.549, .008)\end{array}$ & .22 \\
\hline $\begin{array}{l}\text { Sense of } \\
\text { Urgency to } \\
\text { Screen }\end{array}$ & $\begin{array}{l}\text { Ethnicity } \\
\text { Age } 40-49 \\
\text { OGO } \\
\text { Self-ID as Black }\end{array}$ & $\begin{array}{l}-.667(.33) \\
-.806(.21) \\
.372(.17) \\
.713(.36) \\
\end{array}$ & $\begin{array}{l}0.04 \\
0.01 \\
0.03 \\
0.05\end{array}$ & $\begin{array}{l}(-1.316,-.018) \\
(-1.213,-.399) \\
(.037, .706) \\
(-.001,1.426)\end{array}$ & .35 \\
\hline $\begin{array}{l}\text { Perceived Risk } \\
\text { for Developing } \\
\text { Cancer }\end{array}$ & $\begin{array}{l}\text { OGO } \\
\text { Years in Broward } \\
\text { Ethnicity }\end{array}$ & $\begin{array}{l}.505(.23) \\
-.872(.35) \\
-1.033(.44) \\
\end{array}$ & $\begin{array}{l}0.03 \\
0.01 \\
0.02 \\
\end{array}$ & $\begin{array}{l}(.055, .962) \\
(-1.564,-.180) \\
(-1.913,-.153) \\
\end{array}$ & .19 \\
\hline $\begin{array}{l}\text { Perceived } \\
\text { Capacity to Do a } \\
\text { Self-Breast } \\
\text { Exam }\end{array}$ & $\begin{array}{l}\text { Education Level } \\
40-49\end{array}$ & $\begin{array}{l}-.404(.19) \\
-.447(.22)\end{array}$ & $\begin{array}{l}0.04 \\
0.05\end{array}$ & $\begin{array}{l}(-.779,-.029) \\
(-.885,-.009)\end{array}$ & .14 \\
\hline $\begin{array}{l}\text { Perceived } \\
\text { Benefit to } \\
\text { Screen }\end{array}$ & $\begin{array}{l}\text { OGO } \\
\text { Nativity } \\
\text { Parental Nativity }\end{array}$ & $\begin{array}{l}.402(.20) \\
-1.964(.73) \\
1.861(.82)\end{array}$ & $\begin{array}{l}0.05 \\
0.01 \\
0.03\end{array}$ & $\begin{array}{l}.004, .807) \\
(-3.417,-.511) \\
(.230,3.492)\end{array}$ & .16 \\
\hline
\end{tabular}


Q2. What was the relationship between ethnic identity and health-seeking

behaviors among African-American and Afro-Caribbean women?

$H_{0}$ : Ethnic identity will not have a statistically significant influence on the

health-seeking behaviors of African-American and Afro-Caribbean

women.

Two behaviors represented the construct "health-seeking" for the second research question: (a) likelihood the woman will see a doctor before being sick and (b) likelihood the woman will see a doctor to screen for cancer. Binary logistic regression was performed to explore the relationship of ethnic identity to both behaviors.

\section{Influence of ethnic identity on health-seeking behaviors}

Likelihood to see a doctor as a preventive medical visit. The first model included ethnic group membership (African American or Afro Caribbean) and the MEIM total score represented ethnic identity. Both variables served as independent predictors of seeing a doctor while not sick. This model was not found to be a good predictor of preventive health care $\left[\chi^{2}=3.56, d f=2, p=0.17\right]$. Both ethnic group membership (African American or Afro Caribbean) and a women's s total MEIM score were not significantly associated with the women's behavior to ever complete a preventive medical visit; thus the null-hypothesis of no difference is supported and fail to reject the null hypothesis (Table 11).

A second model was created that entered the two measures of ethnic identity along with the sociodemographic characteristics of the women who would ever see a doctor in a clinic, hospital, or private office before being sick. This act symbolized a woman going to a doctor for a preventive behavior. Having health insurance was the only 
factor that had a significant association with women who would see a physician for a preventive medical visit $[\beta=-2.500, \mathrm{SE}=.76, p<0.01, \mathrm{CI}=(.02, .36)]$. Ethnic identity remained unrelated to a physician visit for preventive health care regardless of the woman's sociodemographic characteristics. Findings continue to support the null hypothesis of no difference; thus, fail to reject the null hypothesis.

Likelihood to ever see a doctor to screen for cancer. Logistic regression was used to examine if ethnic identity (self-described as African American or Afro-Caribbean and the MEIM total score) could independently predict for women to ever screen for cancer. Ethnic identity represented by both constructs was not associated with women screening for cancer $\left[\chi^{2}=51.978, d f=1, p=0.58\right]$ (Table 11).

Model 2, however, found that ethnic identity (MEIM total score) had a statistically significant association after the socio-demographic variables were entered into the analysis (Table 11). Logistic regression indicated three sociodemographic factors along with the ethnic identity represented by the MEIM total score was statistically significant to a woman ever screening for cancer $(\mathrm{p}<0.05)$. Afro-Caribbean women's behavior to ever screen for cancer (referent) was associated with: whether she incorporated holistic and non-western medical services in her health regimen $(p=0.04)$, her income was under $\$ 15,000$ the previous year $(p=0.01)$, and she exhibited an overall positive outlook of her ethnic identity $(p=0.05)$. 


\begin{tabular}{|c|c|c|c|c|c|}
\hline \multicolumn{6}{|c|}{$\begin{array}{l}\text { Table 11: Logistic Regression Models of Factors Associated with Health Seeking } \\
\text { Behaviors among African American and Caribbean Women }\end{array}$} \\
\hline \multicolumn{6}{|c|}{ Behavior 1: Likelihood to see a doctor as a preventive care visit. $(n=117)$} \\
\hline Factor & $\mathbf{B}$ & SE & Df & $\boldsymbol{P}$ & CI \\
\hline Insurance Status & -2.500 & .76 & 1 & $<0.01$ & $(.019, .361)$ \\
\hline \multicolumn{6}{|c|}{ Behavior 2: Likelihood to see a doctor to screen for cancer. $(n=115)$} \\
\hline Factor & B & SE & Df & $\boldsymbol{P}$ & CI \\
\hline $\begin{array}{r}\text { non-Western Medical } \\
\text { Provider }\end{array}$ & -1.934 & .93 & 1 & 0.04 & $(.033, .929)$ \\
\hline Income & -1.677 & .68 & 1 & 0.01 & $\begin{array}{l}(1.433, \\
20.225) \\
\end{array}$ \\
\hline MEIM Score & 1.452 & .73 & 1 & 0.05 & $\begin{array}{l}(1.028, \\
17.743)\end{array}$ \\
\hline
\end{tabular}

To summarize, ethnic identity as ethnic group membership or the MEIM total score did not independently have a statistically significant effect on whether women would seek a preventive health visit. Not having health insurance was the only sociodemographic factor to have a statistically significant effect on the women in this community to ever seek preventive health care. Ethnic identity was unrelated to ever screening for cancer. Use of a non-Westernized medical provider and income below $\$ 15,000$ were associated with not screening for cancer and larger MEIM total scores were associated with screening for cancer.

\section{Relationship of Ethnic Identity and Social Cohesion among Black Women}

Q3. What is the relationship between ethnic identity and social cohesion among African-American and Afro-Caribbean women?

H0: Ethnic identity will not have a statistically significant influence on the detection of social cohesion among African-American and Afro-Caribbean women. 
Logistic regression was used to investigate a possible relationship between ethnic identity and social cohesion. In Model 1 (Table 10), two variables, ethnic group membership (African American or Caribbean; Caribbean women were the referent group), and the score on the MEIM Total scale were used as separate measures to represent ethnic identity. In these analyses, ethnic group membership was found to have a statistically significant yet negative relationship to social cohesion $[\beta=-1.06, \mathrm{SE}=.40$, $p=0.01, \mathrm{CI}(.16, .76)]$. MEIM Total score had a negative but not statistically significant association with social cohesion $[\beta=-.51, \mathrm{SE}=.44, p=0.25, \mathrm{CI}(.25,1.42)]$. This analysis does not support the null hypothesis of no difference; thus, reject the null hypothesis.

Demographic characteristics of the women then were entered into Model 2 for the logistic regression analysis (Table 12). Neither ethnic group membership $(p=0.513)$ nor MEIM Total score $(p=0.190)$ were found to be significantly associated with social cohesion once sociodemographic variables were added to the model. This analysis supports the null-hypothesis of no difference; thus, fail to reject the null hypothesis.

This analysis found, however, significant relationships to social cohesion with three other variables $(\mathrm{p}<0.05)$. Use of a non-traditional provider [does use] $(p=0.02)$ was positively associated with social cohesion while the length of time as a resident in 33311 [less than ten years] $(p=0.02)$ and educational attainment [under college] ( $p=$ $0.03)$ were negatively associated with social cohesion. These characteristics alone created a model that illustrated differences between high and low cohesion, $\chi^{2}=39.03, d f=14, \mathrm{p}$ $<0.01$. 
In Model 3 (Table 12) the variable, importance of identity to defining sense of self, was added to the model to investigate the presence of low and high social cohesion among the women. Model 3 was found to significantly predict social cohesion with $\chi^{2}=$ 43.95, $d f=15, p<0.01$. This analysis does not support the null hypothesis of no difference; thus, reject the null hypothesis.

Four additional sociodemographic variables were found as statistically significant at a $95 \%$ level of significance. Two were negatively associated with social cohesion, [Less than 10 years as a resident in $33311(p=0.02)$ and educational attainment less than college $(p=0.05)$ ], and two variables were positively associated [identity is important to define self $(p=0.02)$, and acknowledge use of a non-traditional provider ( $p$ $=0.03)]$.

\begin{tabular}{|c|c|c|c|c|c|}
\hline \multirow{2}{*}{\multicolumn{6}{|c|}{\begin{tabular}{|r|} 
Table 12: Logistic Regression Models to Predict Social Col \\
and Caribbean Women in Broward C \\
Model 1: Ethnic Identity and Social Cohesion $(n=117)$
\end{tabular}}} \\
\hline & & & & & \\
\hline Factor & $\boldsymbol{B}$ & SE & $D f$ & $p$ & CI \\
\hline Ethnic Membership & -1.105 & .39 & 1 & 0.01 & $(.154, .319)$ \\
\hline \multicolumn{6}{|c|}{$\begin{array}{l}\text { Model 2: Demographic Characteristics, Ethnic Identity and Social Cohesion (n= } \\
\text { 115) }\end{array}$} \\
\hline Factor & $B$ & SE & $D f$ & $P$ & CI \\
\hline Use non-Western Provider & 1.307 & .63 & 1 & 0.04 & $\begin{array}{l}(1.095, \\
12.738)\end{array}$ \\
\hline Residential Time in 33311 & -1.502 & .68 & 1 & 0.03 & $(.059, .837)$ \\
\hline Educational Attainment & -1.250 & .53 & 1 & 0.02 & $(.101, .813)$ \\
\hline \multicolumn{6}{|c|}{ Model 3: Importance of Ethnic Identity to Sense of Self Added to Model 2 (n=116) } \\
\hline Factor & $\boldsymbol{B}$ & SE & $D f$ & $\boldsymbol{P}$ & CI \\
\hline $\begin{array}{r}\begin{array}{r}\text { Ethnic Identity Defines Sense } \\
\text { of Self }\end{array} \\
\end{array}$ & 2.286 & .95 & 1 & 0.02 & $\begin{array}{l}(1.532, \\
63.120) \\
\end{array}$ \\
\hline Residential Time in 33311 & -1.630 & .71 & 1 & 0.02 & $(.049, .786)$ \\
\hline Educational Attainment & -1.055 & .55 & 1 & 0.05 & $(.120,1.014)$ \\
\hline Use non-Western Provider & 1.482 & .66 & 1 & 0.03 & $\begin{array}{l}(1.198, \\
16.182)\end{array}$ \\
\hline
\end{tabular}




\section{Presentation of Phase 3 Data}

Section 1 describes ethnic membership of the discussion groups and Section 2 presents the qualitative findings for Phase 3.

\section{Section I: Ethnic Membership of the Semi-Structured Discussion Groups}

A total of 87 women participated in one of 14 semi-structured discussion groups that were conducted as part of this study. During enrollment participants were assigned to groups based upon their self-described ethnicity (Afro-Caribbean, Haitian or African American). Groups alternated between focusing on ethnicity and health and culture and health (Table 13). This dissertation reports only the findings from four sessions that focused on culture. The decision was made to select the four sessions that contained the most complete set of responses to the original interview guide and represented the ethnic diversity of all of the women that participated in this study (Table 14).

\begin{tabular}{|c|c|c|c|c|}
\hline \multicolumn{5}{|c|}{ Table 13: Description of All Sessions by Topic and Participants' Ethnicity } \\
\hline Topic & $\begin{array}{c}\text { African } \\
\text { American }\end{array}$ & $\begin{array}{c}\text { Afro- } \\
\text { Caribbean }\end{array}$ & $\begin{array}{c}\text { Mixed } \\
\text { Ethnicities }\end{array}$ & \# Sessions \\
\hline Culture & 25 & 14 & 4 & 8 \\
\hline Ethnic Identity & 21 & 5 & 0 & 5 \\
\hline Triangulation/Recap & 7 & 10 & 0 & 1 \\
\hline Total & 53 & 29 & 4 & 14 \\
\hline ed ethni & resents wo & dentify as & $\begin{array}{l}\text { American an } \\
\text { Ethnicities w }\end{array}$ & $\begin{array}{l}\text { ro Caribbean } \\
\text { self-described }\end{array}$ \\
\hline
\end{tabular}

\begin{tabular}{|c|c|c|c|}
\hline \multicolumn{4}{|c|}{ Table 14: Ethnicities of Women in Selected Sessions for } \\
Dissertation (n=4)
\end{tabular}




\section{Section II: Qualitative Findings for Phase 3}

Themes were identified from the four transcripts that aligned with four primary areas of interest in this analysis: (1) describe women's perceptions about mammography and breast cancer, (2) describe how women characterize culture, (3) explain how ethnic identity and culture influence the health-seeking behaviors of African-American and Afro-Caribbean women, and (4) list recommendations made by the participants to improve women's use of preventive care and cancer screening services in their community. Explanations, themes, and recommendations were operationalized from the perspectives of the residents of the community. Comments were contrasted by the ethnic labels of the four discussion groups (African American or Haitian). The relationships and perceptions that the women suggested influenced their health-seeking behaviors were organized using open codes. The results are summarized as general impressions across the entire sample and then described separately for African American and Haitian women.

\section{Description of Women's Perceptions about Mammography and Breast Cancer \\ Expectation of pain. Most women, both African American and Haitian, stated} that they had experienced having a mammogram. They were asked to explain their first experience having a mammogram. Women who had completed this exam fell within one of three categories: (1) had an expectation of pain before the exam, (2) experienced pain, and (3) experienced no pain nor had negative expectations before the exam.

Many women who had an expectation of pain their first time attribute that expectation to rumors they had heard from other women. No one described literature, brochures, etc. as a source of information about the screening exam. They primarily 
obtained information about the exam by word-of-mouth. Interestingly, five women reported that they had never completed a mammogram. Three of the five women in this category suggested that the expectation of pain influenced their decision not to have this exam. Other reasons included one woman admitting she did not know why she had not received a mammogram who later explained it was fear as well. Another, woman responded that she anticipated completing the exam "eventually" but she had not experienced any symptoms so had no sense of urgency to complete the exam. There were a few women who also talked about having completed a mammogram in the past but were no longer compliant with screening recommendations. These women suggested that fear of cost, apprehension related to a bad experience and intolerable pain, physical injury to their breast, and insulting comments from the medical providers were explanations for not maintaining their compliance. An observation was made of the women who had not experienced a mammogram during the discussion groups. These women would ask direct questions of the other experienced women regarding their experiences with pain as if they were validating the accuracy of their preconceived expectations about pain.

Pain was explained by three commonly held perceptions: (1) the size of the breast determined the presence of pain, (2) the skill of the technician determined the experience, and (3) use of breathing and meditation techniques could overcome any discomfort during the exam. The women who had completed the exam offered these tips to the women who had not completed a mammogram. These women also used humor to persuade women to make a commitment to complete a mammogram in the future. Few of the women stated that they did not experience any type of discomfort or pain. Some 
women had dismissed the pain as a quick inconvenience and necessary to stay healthy.

While some who had experienced pain had delayed repeating the exam based on their

painful experiences.

It depends on the physician doing it, you understand? It's never hurt me either.

But depending on the person, doing it for you maybe...they might pull it too much, press the machine too much on it. But there are people who good take care...so it depends on the person doing it. (Haitian woman)

Sometimes it hurts, when the breast are big. Depending on how they place it and it hurts. There are actually people who do it...I've had it done several times, and it didn't hurt. But there was this one time, where I had gotten it done and it actually hurt. (Haitian woman)

My experience is different, the few times, that I've done it, it's really painful, I always wonder...Maybe you should try that, if they could huh...how do you call it...

Yeah helpful and painful, but like our experiences...I don't know now, because when I was in Haiti, I didn't hear that you had to have mammograms. (Haitian woman)

Participant: What it did to me, it made a-pinched it right here, and it pinched me right there. And it shows right now.

Participant: You still have bruises?

Participant: I still have bruises.

Moderator: Right, so how-again, how long ago was that when you went?

Participant: About five years ago, and I ain't been back no more because it messed my breast up.

Participant: So you haven't had an exam in five years?

Participant: No. (African-American women)

I didn't like how I felt either because you know, it's a table, you know, and they squash your breast, all laid out, and you're like this for a minute - can't breathehold your breath. But I'm just glad-because when I was - (African-American woman) 


\section{Women Characterize Culture}

The following five themes reflect the women's definitions of culture as presented by the study participants. These themes applied to both African-American and AfroCaribbean women.

Food Represents Culture. Common to all of the women interviewed for the dissertation is the importance of food in their lives. Beyond the basic need of nutrition, food symbolizes the character and history of their family, ancestors and community. There is pride among the women to share information about a dish and highlight that a meal such as peas and rice while tailored to the ingredients and experiences of a particular country or region is a universal dish shared and glorified by all Black women. The women mentioned that the transportability of food from one city to another reflects their ability to maintain the closeness of their family and roots even when they have moved to foreign areas. The ability to carry out the tradition of the meal and the dishes incorporated in the meal is ceremonial in nature and similar to homage to the women's ancestry.

It's also our heritage.

Ours is... We are an influx of ...they say New Orleans is the northern most Caribbean city. Our food is more of an influence of Haitian identity than European than anything else. Then umm its French African German it's a bit of everything that's why they call it the gumbo state. The gumbo and the people we are a very select breed of people. very select. (African-American woman)

and then food like over there we have Johnny Cake but over here they call it hoecake there you know we have pigeon peas and rice and like a lot of people say they don't like the pigeon peas and rice they like red beans and rice but we like pigeon peas and rice. And a lot of things are pretty much the same [ummhmm] and to me when they speak of culture that's what I think of ...(AfroCaribbean woman)

The food. Our food! Yes our food... [You would say your food is the most important part?] Yes our food is very important(Haitian woman in English) 
Dictates code of conduct. Culture is directive and sets the norms of behavior in the lives of Black women. Culture acts as a code of ethics for how the women will approach their daily interactions by acting as a reference to select socially acceptable behavior. The strength of culture is viewed as the mechanism for maintaining social respectability among the Caribbean women while the absence of a strong cultural bond is suggested as a reason for why poorer health outcomes remain prevalent among African American families. African American women suggest that reconnecting to cultural values and norms will improve their health. Haitian women explain that the migration of Haitians from their cultural norms is responsible for their adoption of drug use, crime, violence, and other behaviors associated with being an American.

Everything... everything we do is ... Everything is by culture. We do everything by culture. The way we eat. The way we dress. The way we raise our child it is all by culture.(Haitian woman in English)

(speaks in Creole) Haitian culture for me... is how you present yourself, what you do as a Haitian woman, how you live. How you present yourself as a Haitian woman regardless of where you are. If how you uphold your culture, there are certain things that you don't do. Per say for a Haitian, you feel as if even though drugs are taking over the country, because of the fact that you came from Haiti during our time...there was a higher status, if someone went to prison, it was something that was seen as low, a state of conduct that you shouldn't be in. If a person steals, you would feel as if that's very low. So you have to uphold a selfprestige, to be able to feel that all of this is apart of your culture. (Haitian woman in Creole)

So in the beginning, we were talking about culture, and you know that in Haitian culture, for a lot of people, the first thing that they do isn't going to the doctor. They go to a witch doctor, they drink tea; they do different things with plants, before they finally go to the doctor. That in itself, is something that we normally do in Haiti. In Haiti when we're looking at our health, we do a lot of things with plants. Plants are really important to us, in the Haitian culture. (Haitian woman in English)

When it comes to culture, culture is something that wasn't created. It was something that's learned. It's a learned behavior. So, with that being said, meaning that there's millions - hundreds of millions of different cultures as a world, as a nation - as a nation. It's not just only - it all depends on once again, 
where you're from, and you know, not necessarily who you are, but where you are in terms of learning about your culture, learning what's important in your culture.

Now you have black Americans - we didn't go to the doctors. Those white folks didn't take us to the doctors when we got sick, so we knew nothing about prevention. We knew nothing about what not to eat and what not to do to not set on diabetes and high blood pressure. We didn't know how to go get screened for heart diseases. We didn't know. So because we didn't know, we didn't grow with those trends to educate us to say - it wasn't a household discussion to say, "Hey, I'm feeling sick. I felt a lump in my breast." It wasn't discussed. So whereby other cultures in different families, and different ethnic backgrounds, those were top priority conversations at the dinner table. (African American woman)

Culture creates connections and cohesion. Culture has the ability to bind individuals and unify people within the community. The participants describe the family unit as a central fixture in their network where immediate and more distant relatives can come together for support, guidance, and rejuvenation. Their expectation is that within the family one is protected, but one woman suggests that the family may not be the supportive network she expects. African-American women describe a central figure who is viewed as the matriarch or elder and this person receives complete authority of the family and sets the tone for the normative behaviors related to conduct and health. AfroCaribbean women reference their pastors with this authority.

Mine is more principle. Coming from a black family, we were brought up onraised up on certain principles. And that binds us together. Where she says unity, the principles are our unity. It binds us together. (African American woman)

Even though we may argue from time to time, but we love. It binds us together. It goes back to what was instilled in us, and we instilled it in our children.

My father had some grandchildren ranging from the age of 25 to 60 something. So we have a certain principle that he instilled in us that binds us together where it can't be broken. So that's coming out of the black culture of family of the descent of where he was raised from coming out of Georgia and with his family through all of this siblings. We're bound together under that same principle. (African American woman) 
Faith is an essential component of culture. African-American and Haitian women had similar perspectives about the church and faith playing a prominent role in their culture. This role has led to a great deal of credibility and trust among clergy as leaders. There was a difference, however, between the two groups in what they expected from their clergy. African-American women made comments that suggested that pastors were considered leaders in their community and their roles as leaders included actively initiating events that would contribute to women being healthier. Haitian women, however, suggested that a priest's role included the ability to sanction behavior through the encouragement or invitation provided from the pulpit. In this case, the pastor's voice to encourage their involvement in screening is the most effective tool to influence Haitian women's involvement with mammograms. Without their invitation, Haitian women would be less encouraged to complete the exam and communication on breast cancer would not occur.

So I think a lot of these ministers can do better with their congregation to get them to really seriously consider getting into the doctor, getting the understanding of it, especially the older ones. (African American woman)

And like she was saying about the congregation and the ministry, instead of them getting a busload going - I mean, it's okay to go to a spiritual retreat, but get a busload and take them down to go get a breast check or educate them how we're getting educated about. Like so fast they want to sign you up, and pay money, and let's go here for the weekend. Let's get a busload and let's all go to the clinic or something like that. (AfricanAmerican woman)

In our community those people they believe in the priests and their pastors. The only way they can do it is by talking to the priests and the pastors so they can talk to them. So they can do it. Interviewer: So they are the most important gate keepers?

Yes. (Haitian woman) 


\section{Greater trust in holistic options over medical approaches. Women were}

empowered by the perception of self-care through diet and nutrition. African-American and Haitian women shared a common cultural trait whereby current attitudes and behaviors were molded by their elders. Uses of plant-based and "natural" remedies were learned behaviors that had been instilled into families over generations. Many AfricanAmerican and Haitian women were exposed to the practice of using food and natural remedies to manage their personal and family health by their parents and grandparents. Several African-Americans described a sense of comfort in their familiarity with using these strategies and perceived validity due to repeated positive outcome with these strategies in caring for themselves and family members. Both groups perceived these approaches as tested and proven methods.

A lot of them don't think about their health. They just eat poorly that's why you got a lot of obesity and a lot of strokes and heart attacks, too.

Participant 5: I don't totally agree with that, and when I say that, I do agree with poor eating habits. A lot of people in our community don't go to the doctor at all. So I really agree with that. But you have to think about - and you can attest to it being from a large family, your older siblings, what we were able to eat has drastically changed because my grandmother would drink a little bit of rum. She had collard greens and ham hocks.

They ate fat pork, salt pork. She'd put it in the food. So that part of me didn't change. But what was different, you didn't have the genetically modified foods that we do now. So they planted their own-my aunts and uncles still go to the back and get their greens and stuff like that. They didn't rely on these huge companies that use pesticides and all of these things. So it's not just our diet. It's what they're putting in our foods now because we ate more - we weren't able to afford steak. We ate a lot of chicken and stuff like that. So we did more vegetables, a big thing of rice and beans.

Participant 3: So it's what they're putting in them animals now. 
Participant 5: Yes. That's what I would say. It's affecting what we would culturally grow and eat. It's affecting this. (African-American women)

\section{Influence of Culture and Community Norms on Health Behaviors}

The sixth research question addressed the ways in which culture has influenced the health-seeking behaviors of ethnically diverse Black women. The previous section highlighted the characteristics of culture that both African-American and Afro-Caribbean women viewed as important and essential to their cultural identity. This section attends to the expressed characteristics perceived by the women as unique to their ethnicity.

\section{Culture and Community Norms for African American Women.}

Two themes represented the community norms that the participants perceive have influenced African-American women's health behaviors. These themes included (1) adoption of holistic preferences and (2) leaders influence health behaviors.

Adoption of holistic practices as a community tradition. Women discussed the migratory history of the community highlighting the pipelines where the majority of the population had originated. Most families originated either from the lower southern states of the US or from the Caribbean islands, namely Alabama, Georgia, Mississippi, Carolinas and the Bahamas. In these sessions women talked about the various lifestyle behaviors that had originated from these points of origin, were adopted by their families, and subsequently adopted as tradition. Among these, African-American women commonly used plant-based medicine, consulted with the "root man" for the medical needs, and drank cerasee tea to remain healthy. Women talked about over time these behaviors had been adopted by many families as the standard of care. Many of the 
behaviors have remained ingrained in the community. As such, a number of women

spoke about integrating these behaviors in their health care regime alongside

conventional medical practices. Many women preferred to use natural options and food

to take care of their health while a few abstained from engagement with the conventional

medical center altogether and only used holistic strategies.

So we were our own physicians a lot of the times. So it gets back to the cultural basics. Like I said, you guys have that history. I have that history only because I knew my great grandmother. So I knew about the remedies and things like that. So I don't have any pills, nothing, no Tylenol, nothing in my house. And it's that kind of culture that we I think lost. And that's where I think the health problems came about when we started relying more on manmade pharmaceutical drugs and not healthy alternatives that we used to do. So a lot of things that I think we don't go to the doctor because our grandmother didn't go to the doctor so much. (AfricanAmerican woman)

Leaders influence health behaviors. Elders in a community and in the family set the tone for embracing health options. African-America women indicated that the elders in their families commanded the respect of the entire family. In this regard, they became the primary source of cohesion for the family and they established the rules of engagement for the family including behaviors that were sanctioned as the traditions of the family. This included dictating the norms for managing children's health, setting priorities and expectations of family behaviors, and endorsing the mechanisms that members use to support each other. Their influence had an intergenerational effect whereby health practices of great grandparents were often maintained two to three generations later.

...my family has been here since the 40 s, entrepreneurial family. We did have a matriarch, and my great grandmother died at 102. All of her kids are still living. The oldest is 96 now. So we do get together for her 
birthday every year. We kind of made her the next matriarch, even though she didn't want that position. So we kind of forced ourselves on her. With Christmas, everyone goes to her house like we did my granny. So with that, I can understand now who is going to lead after her is the scary part because, like she mentioned, there's really no one else at that level that commands everyone's respect. (African-American woman)

Okay me my culture it's between three different groups. African American, American Indian and Bahamian. The two likeness between the Indian and the Bahamian is the medicine man. like roots. Herbs. That's the same thing as American Indians that side my grandmother's side was into a lot of rotted woods and find something for a toothache. Belly ache...skin. Same thing with my dad's side the Bahamian. Once a month we had to line up we all got lined up to get a cup of cerrasee and a spoonful of sugar and you were never sick [laughter](Ethnically-Mixed woman)

\section{Culture and Community Norms for Haitian Women}

Three themes represented the community norms that have influenced Haitian women's health behaviors. These themes included: (1) Faith as an essential characteristic of Haitian culture, (2) Class, income, and immigration status intervene with healthseeking behaviors, (3) Preventive care differentiates Haitian and US health care systems.

Faith is an essential characteristic of Haitian culture. Faith speaks to both the internal belief of the women in their God and God's omniscient power, as well as, to the institution of religion, which includes both the church and its religious leaders (i.e., the priest). Faith in God was perceived as the most important characteristic of Haitian culture. Their belief in God has been passed between generations and its historical significance to offer support, empower and comfort in positive and negative instances is a universal characteristic of most people of African descent who practice the Christian faith. These participants expressed similar opinions that faith was an endearing quality of their culture and had a personal importance for both groups. The church was also 
perceived as a vital source of information to the immediate members and community atlarge for both groups. Related to health care, women described the church as the perfect venue to conduct health outreach and the church leaders, particularly the pastor, along with cancer survivors as the best identities to conduct outreach in their community. If you know Haitian culture, you know, we're one of the first black republic independent in the world, you know and our culture is based on you know, not...it's based on religious background really. So, I mean, I know we have different types of religions, denominations but mostly it's you know Christianity, we take pride in that, we take pride in that. Hmm you know we have a rich history, nobody talks about it, nobody knows about it, but we have a few, if you go do your study, we have a rich history you know. Now unfortunately, it's all going down but, we still take pride in being Haitian. (Haitian woman)

In our community those people they believe in the priests and their pastors. The only way they can do it is by talking to the priests and the pastors so they can talk to them. So they can do it.[So they \{priests and pastors\} are the most important gate keepers?] Yes. (Haitian Woman)

Really work through the churches and get the pastors be the voice to encourage their involvement in screening. (Haitian woman)

Belief in God's ability to manage a person's health status has discouraged some Haitian women from participating in the medical system. Similar to the concept of fatalism, these women believed that God's will for a person to be healthy or sick superseded any medical intervention that would be performed by a health provider. Therefore, it did not make sense to screen for cancer or to engage in preventive health care services. There were a few African-American women who also held similar views, but overwhelmingly this perspective was expressed by Haitian women.

I have faith in my God to take care of me. (Haitian woman) No. I won't go. Why go? If God wants me to be healthy then I'm healthy. (Haitian woman) 


\section{Class, income, and immigration status intervene with health-seeking}

behaviors. Haitian women described their experience in Haiti to obtain health care. The discussions emphasized that their country has always been challenged to provide acceptable care to the majority of people who lived in Haiti. The limited availability of medical facilities had contributed to this problem despite the fact the skilled providers were available in their county. As such, access to the private clinics and hospitals and even some of the public care facilities was challenged by your ability to pay for services. Unlike the US, health insurance was not available in Haiti.

The women explained that their circumstances of living within a low-income country created the perception that access to medical care was dependent upon your wealth. Participants explained that poorer residents were directed to certain facilities where the expectation of mortality was high, and the availability of essential resources was low. As a result, a large portion of Haiti's residents turned to plant-based medicine to obtain medical care. As with many African Americans, this behavior was institutionalized as effective care and, because plant-based care had become the primary health care option, people trusted plant-based medicine over public-care facilities. These perceptions influenced health behaviors in two ways. First, participants explained that most women currently living the community were socialized to use plant-based care options. Relatedly, many Haitian women, like themselves, would be more receptive to "natural" options, such as breast self-exams, than mammograms to monitor the health of their breasts.

...but health you know, we always love our teas you know our herbs you know, for the high blood pressure you have that herb, for the sugar you 
have that herb, for the cholesterol...So we mostly herb oriented culture than medical. You agree with me? (Haitian woman)

Yeah, we mostly herb oriented, you know than medical. I mean, I know I am. (Laughs) (Haitian woman)

To do preventive medicine in Haiti, you would have to have money, you know. So not every...I mean we have... Haiti is like compartmentalized you know. You have the rich rich rich, you have the so rich, you have the middle, you have the after the middle, and then you have the poor poor poor poor. Not everybody has access to you know medical care, medical health you know, medical... or a hospital. They don't have that kind of money. So the people that are rich, they come here for preventive medicine you know. They set up their schedule, come every year to get the check-up you know. But in Haiti you don't do preventive medicine, it's...so when it happens then you go to the hospital if you can get cured then you know. You know in Haiti, sometimes, I don't know if it still happens but before because I used to help, you have to bring your own gloves to the hospital, your own cotton swab, your own huh...everything! You have to bring it to the hospital, so who has time to go to the hospital for that? So they, they just drop dead. (Haitian woman)

Accessibility to care as a poor person has presented unique challenges for Haitian women who currently live in the US. One woman addressed a perceived bias that she has experienced as an uninsured resident. Her comment suggested that the local hospitals had not been welcoming facilities to residents without insurance. Her observation also implied that the quality of patient care was based upon the patient's ability to pay. If this were commonly held perception by a significant number of Haitian women, her experience offers some clarity for why many Haitian women may have avoided healthservices at this institution.

But who decides if it's life threatening if they don't really do it deep. Because I know people, that have gone to the hospital and because they didn't have insurance you know, they just give them something, and when they get home, you know they get worse, or they die. So...(Haitian woman)

Yeah! They ask you what's your insurance, who's your carrier, and if you don't have one, they don't really care about you.(Haitian woman) 
You heard about it, but in my age, we didn't hear about mammograms, we didn't hear about pap smears, so it's a plus. But still, I don't think, it's as accessible.

Preventive care differentiates health care systems. The ZIP-code 33311 vicinity has always been the first port of call for people of African descent who migrated to Broward County. Haitians have been challenged to adjust to the norms that dictate the health-care system in the US. The women first explained that the idea of prevention was foreign to women from Haiti. Haitian women were socialized to see a doctor only when sick; therefore, a preventive visit was counterintuitive to many of the Haitian that lived in this community. Moreover, medical care was perceived as an entitlement for individuals who were upper class and not a privilege of the poorer class. Most of the Haitian women living in this community represented the less affluent. Therefore, most of these would have had experiences and perceptions similar to those being described in the discussion groups.

The way we define health. It's like ... Like I just said if you not sick you're not seeking care ...medical care. Compared to the American culture they are seeking care on a month... on a regular basis. For us if you're not sick you're not going anywhere. [So the idea of what we call a regular basis....] for a check up for a regular basis... [So that is what we call an idea of prevention] ...prevention yes [so the idea of prevention is not necessarily part of the culture]...part of our culture (Haitian woman)

In my opinion, in Haiti it's when you're sick that you go to the doctor. It's rare for you to wake up and say that you're going to the doctor even if you aren't sick. Just for a checkup. (Segment completely in Creole). There's nothing like that. Here, you go, you check-up, you have either yearly check-ups or if you have your private doctor, even the the...the 7th Ave, you have your... appointment every four months to go to check the doctors. Even though, even if you're not sick but you will have to go. That's totally different but in Haiti, you have to be sick and really sick, after you've taken all of the herb medicine, if they don't work then you go see the 
doctor. (Other women agreeing in the background). So this is very different, that will make you feel comfortable going to see a doctor, at the same time some people would feel reluctant to go because they will be more relying on the herb medicine than going to a doctor. Because they say well, I spent all my life without going to the doctor why should I have to go every three, four months okay but that will help. This is why certain people in our country in Haiti will die; we don't know what killed them because they don't consult a doctor regularly. But here, we have the prevention when we go, we have, we can check-up for example our breast, we could check, we could know that there is something that's building in, we will know the prevention. But back to Haiti, people will drop, drop dead but people we'll say well they're something, voodoo that they did that killed. No! High blood pressure, sugar, something else, because they did not prevent that. So this is the difference between here. Here yes, we will go and even though if you do not have money, you will find it huh...some help assistance from the government. In Haiti there is no help from the government, even though you go, even though...if you go to the...public hospital (other women saying it too), you still have, if you don't have money to give, they will not even look at you. So that's a big, big difference between those two countries. (Haitian women)

More importantly, several comments were made by Haitian women that receiving mammograms were not routinely practiced in Haiti. Arrival to the US for many was, admittedly, their first exposure to mammograms and other gynecological services. Given that many of the participants have lived in the area at least 10 years and all were at have least 40 years of age they attributed their lack of awareness to a generational difference.

...I don't know now, because when I was in Haiti, I didn't hear that you had to have mammograms.

That's right you didn't know that. (Segment completely in English)

I didn't know but now I said, when I was in Haiti, I didn't hear about mammograms, or pap smears... (Segment completely in English)

We didn't have the prevention. That's what I said...(Haitian women) 


\section{Culture's Influence on Women's Health-Seeking Behaviors}

Two themes were established to describe culture's influence on women's actions to engage with their doctor for preventive medical care or to screen for diseases such as breast cancer. The themes highlight culture's influence on the decision making of women to complete these activities with their doctor. In addition to the two themes four concepts (fear, motivation, pride, and need for support) are highlighted as mediating factors that influence the women's decisions to complete the medical visits.

Women have conflicting views on necessity of screening. Individuals and family have remained healthy without these services. The first viewpoint contends that medical visits were not a necessity because the individual and family members have remained healthy without the use of preventive visits. In general, absence of symptoms of illness (i.e., pain, bleeding, etc.) translated to the woman that they were healthy. This sentiment was expressed in varying degrees in all four sessions, but Haitian women most emphatically expressed that symptoms signal the need to see a doctor, and without these symptoms, the women did not seek a medical visit. A few African-American women mentioned that a person could be sick yet never show signs of illness, fewer Haitian women made this reference in their groups.

Yes, I'm healthy by not being sick. I don't have to go to the doctor. I don't have a headache. I don't have a stomach ache therefore I don't need to go to a doctor because I'm healthy. (Haitian woman)

Because we don't do check-up we don't know maybe when we get to the doctor maybe it's stage four we don't screen before we don't know unless it starts hurting. Unless we in pain that's when we seek care so when we go it might be too late and then there no there's no nothing we can do about it. (Haitian woman, English language). 


\section{Women understand importance of prevention yet refrain from care. The}

second viewpoint was that some women understood the importance of preventive medical visits but had made a conscious decision not to see a doctor. There were several reasons given for why women made these decisions. First, many women had competing priorities and these issues took precedence over seeing a doctor for any reason.

Preventive visits became less important because of the urgency or immediacy of the problem. These issues could be personal, related to other health concerns, and external to family and community members. None of the comments made by African-American or Haitian women suggested that this community prioritized medical visits. Thus suggesting women in this community did not prescribe medical visits as something that women must do.

Yes screen for breast cancer...you know I have high blood pressure, high cholesterol, I have to take care of my kids, I have so much ... so I just can't think about my breasts (Haitian woman)

That's difficult to say because sometimes we can know and still won't go...[why?] I think it goes to back to your culture [laughter] (Haitian woman)

I've had my sugar (?) for about two months I still haven't go yet.... (Haitian woman)

Fear can mediate behavior. Fear as it relates to the disease to the possible social consequences associated with discovery and disclosure represented the third viewpoint for why women were not screening. Fear has caused some women to avoid these exams. Some women would not go to the doctor even though they were aware that avoidance increased their personal risk for developing medical issues. Some women were afraid of 
hearing the words "you have cancer," some feared the pain that was rumored as a part of the exam and, in others, fear turned to denial about risk for developing the disease.

Fear was also associated with an anticipation of physical harm from exposure to the examination tools (i.e, bruising and pain from the mashing of plates). This particular fear had two origins: (1) rumors shared between women about the examination, and (2) trauma from a personal experience with the exam, prompting the woman not to participate in follow-up exams.

Fear of the unknown for women who had never had a mammogram have led to anxiety and avoidance because they are concerned about cost and pain. These women asked other participants who had completed the exams about their experiences to get information that may confirm their expectation of pain during the groups. These fears were found among both African-American and Haitian women. Finally, dissenting opinions among African American women presented fear as laziness and that women's avoidance was a function of laziness rather than fear.

I was scared [and what exactly were you scared of?] I don't know because of the pain. [snicker] [when you made a child... who got more pain?] [laughter](Haitian Women)

Interviewer: Is there any other type offear that's involved in that decision?

Participants: Sometime you worry about what if they find something, what are you gonna do? [Can you speak more on that]

But you have to know. You have to know... [that's why you do it so that you know... to see if something wrong or not. They check you out it's okay if not okay they tell you what to do you go the doctor to see what's causing it.] Yeah you have to know.... (Haitian women)

I had been scaring myself to death because-

Because you don't know which lump is cancer... (African-American women) 
Well I think I did hear about that ...there was a woman who was so afraid about getting it she went around because it was in her family that she was gonna get ohh Im gonna get it Im gonna get it ...then she finally did find out she got it and she was almost like relieved. It was like a big ton of weight off of her so she was like well now I know I got it so she could move on.(African-American woman)

Participant 5: I would say this in addition to what you ladies are saying and that's that the $C$ word is still very terrifying for people.

Participant 3: Yeah, it is.

Participant 5: So I've had family members, I had my dad pass. He got diagnosed with cancer. Not breast cancer, but he got diagnosed with cancer, was telling everyone he was going to chemotherapy, wasn't. And the doctor calls me like we haven't seen your dad in four months. What do you mean? But I had a conversation with him. He says, "If I got it, I got it. I don't want to know." And that was his attitude. And I have his brother is the same way.

He refuses to go to the doctor to get checked because it runs on my patriarchal side. And he's like if I got cancer, I don't want to know. So you have some people still today, believe it or not in 2014 that still have that mindset that the $C$ word is not something they want to confront because they look at it as a death sentence. And even though it's not nowadays, especially with breast cancer, so you do have women that I don't want to know because if I take that exam, I may get that result that I don't want. So I'd rather not know. (African-American women)

And you know some people sit and think cause my sister died of breast cancer I'm going to die of it...I didn't want to call your name...you know? That's probably why a lot of them really don't want to know.

[So thinking like because my family passed then I'm more likely to pass from this too so I just don't want to know...??]

Umm hmm yea they just don't want to know.

Is it an idea that it will prevent them from being sick by not knowing or... just don't want to know and you can't make them .... (African-American woman) 
Motivation can mediate behavior. Women in this study offered examples of factors influenced their decisions to maintain their health status. Some woman explained that their responsibilities to care for others in their family were linked to their health status. Maintaining good health provided them with the capacity to perform their obligations as a caregiver. As a result, these women were motivated to screen for cancer and to remain healthy. Relatedly, some of the women discussed the concept of self-love as a second source of motivation to engage in preventive care visits. This discussion suggested that women with a strong sense self-love and sense of personal importance would want to take care of their health and engage in preventive health behaviors. African-American women offered these explanations as they related to their roles as caregivers.

Haitian women also mentioned a similar term, self-respect, as a source of motivation to see a doctor and screen for cancer. One group explained that Haitian women traditionally took care of themselves as a matter of self-respect. This relationship transcends to dress, behavior, health, and the manner that you treat others. For African Americans, being healthy motivated the women to fulfill their role as caregiver. For Haitian women being healthy was motivated by the fulfillment of an expected behavior within their culture. These discussions identified areas that may influence health-seeking behaviors and cohesiveness in this community.

...yeah, I just wanted to add to that, a lot of us just not interested in going to the hospital, to the doctor. We don't-it's not even a learned behavior. It's just the way you feel about yourself, you know what I'm saying. If you feel important enough, then we go see - well, I feel something right here; I need to make an appointment. (African-American woman) 
And they gonna assign you one, too, because-excuse me - I started going to the 7th Avenue clinic because I heard through word of mouth, I got the information to go to them because I didn't have any health insurance, like this was like two years ago when I found out about it. So guess what, the first thing I did, I made my visit to go there. I made an appointment. I went down, waded through the process, everything that they had me do. And I got it done. I went and saw a doctor. That's because I cared about myself and my health because I have three kids to live for. (AfricanAmerican woman)

Pride can mediate behavior. Pride has been attributed the health-seeking behavior of some women. The participants offered that personal pride explains why some women did not ask for assistance from the medical establishment, family members, and the community when they were ill and needed to see a doctor. Pride was associated with feelings of embarrassment. The embarrassment would result in women who needed help but remained silent about their condition and did not seek assistance. Women also chose to maintain their silence because did not know where to go for help in the community and delay or refrain from asking for help from their family members and friends. Some people also saw illness as a weakness or vulnerability and would avoid the health system because in the admission she would place herself in a vulnerable position.

Participant 3: Some people, and they have that pride about themselves that they won't even seek help.

Participant 1: That's laziness.

Participant 4: It is.

I just think it's people in general. They just don't have that sense of pride. And they don't ask for help.

Participant 3: And they can't afford it, and they won't ask for no help. And a lot of stuff is free, but they just don't take the initiative to get it. The clinic was here for a lot of years now. (African American and MixedEthnicities Caribbean women) 
Pride, in contrast, has been a positive influence on the health decisions of Haitian women. Their legacy has evolved into a collective sense of pride based on the notoriety of their country as the first black republic in the world, and the second nation in the western hemisphere (after the United States) to win its independence from a European power. The women explained that their culture and underlying community norms were dictated by this perception of pride. Therefore, Haitian women maintained their dress, appearance, behaviors, and personal health in response to a historical responsibility associated with their heritage. Their descriptions of an "old and new Haiti" explained their perception that cultural pride has diminished resulting in an increased number of Haitians who have adopted behaviors such as illicit drug use, violence, and change of diet. These behaviors were contrary to their behaviors norms. Therefore, a person who has maintained their ethnic-pride would be associated as a person who takes care of their personal health.

Support mediates behavior. African-American women indicated they need more support from family, friends, and church leaders to improve their health. Women described some of the difficulties that they encountered in trying to improve their health. One participant discussed that aging has made it more difficult to be motivated to take care of one's health. Comorbidities such as diabetes have reduced mobility and the quality of life for many women who live in this community. Often addressing problems such as diabetes and high blood pressure were more immediate and taxing. There was not enough inner-strength to potentially add breast cancer. Therefore women delayed or avoided having a mammogram and investigating their health status with a doctor. More education and residents teaming up with each other to improve their health were 
examples of support that African-American women needed in this community to promote more engagement.

Even though my mother, she was very cautious to raising, growing her food, making sure that we ate right, had a balanced breakfast before we'd go to school and so forth, she made sure of that. When we came home, there was always a snack for us. And she was good about that. But it was like they, in a sense, were ignorant to other things, especially when it came to their health and watching out for themselves. That's why they deteriorated so fast, especially with the diabetes. (African-American woman)

One-on-one support by accompanying a woman for her mammogram was suggested as a solution to make women feel more comfortable with their medical visits. As women's comfort levels increased, so were the chances that women would sustain these activities. This support also served as an immediate resource for encouragement if cancer or other health issue was diagnosed during the medical visit.

Tom Joiner has a good thing that kind of started where [to] take a loved one to the doctor day or what have you. So I think perhaps if you've gone - I know I've taken the exam. If you've gone, you just kind of express it's no big deal. It's five minutes, and you're done.

Participant 2: Support is the biggest part of it. You need support. (African American Women)

I think it should be pushed on a regular Sunday basis in the congregations because people are really not getting out and doing it. And with our culture, black people, it's just hard to get us in a doctor's office.

I noticed that even with my neighbors, and I joined the $Y$. And going to the exercise classes and going and learning all the different healthy living classes, I can't get people out to support it. I couldn't do it. I tried to advocate that in my neighborhood. And it just didn't work just getting them to get out to the classes. These are retirees, older people doing nothing. And not that I needed the support, but you need it to know. You need to know what to ask your doctor. You don't need to go to your doctor and leave there not knowing what he just told you, not letting it sink in to the point where you're going to do better with your health. 
So I think a lot of these ministers can do better with their congregation to get them to really seriously consider getting into the doctor, getting the understanding of it, especially the older ones. (African-American woman)

Support became a mechanism to promote continuity with the healthier behaviors.

As one women explained, often "people improve their health habits but relapse."

Testimony from one participant explained how her support group enabled her to recover and sustain her health behaviors.

So you know I was eating. So done gained all this weight. And so now, every morning, I go out and go walking. And I tell my boyfriend, he's 66 years old, he's a Jamaican, and he eat real healthy. So I find myself eating healthy because we got a little garden in the back. But if I didn't encourage myself, who else would do it? And so I try to get him, "Come on, baby, let's go for a walk, " because he had a colonoscopy, and they found a little thing on his prostate. And now they found a big knot on his kidney. But he's Jamaican, and he's stuck in his ways.

Interviewer: So can you explain to me how that then - when you say stuck in his ways.

He's stuck in his ways because, at first, he stopped taking his medication. He want to grow herbals and all that other stuff. And when the herbals didn't work, and he's starting to get sick at night time. Oh, you got to take that manmade medication now. So he has Humana. Humana offers you to go to the gym. I try to get him to go.

... Take me with you. Let's do this together. I don't get none of that. I don't get none of that. So like I said, it comes from-you need support. And just like they said, when two or more people pray, he's always in the midst. It takes more than one person. It takes more than one person.

(African-American woman)

An important characteristic of "support" was communication. Haitian and

African-American women wanted more communication about health and cancer in their social circles and the community at-large. A few comments were made that questioned why people do not hear the statistics about cancer risk, prevalence, mortality, survival, and actions that can prevent disease and mortality in the community. There was a call for 
more education and this education should be provided to older and younger residents. All four sessions explained that an effective educational platform should be straightforward, transparent, graphic, and include risk as well as strategies for survival. Education is also needed to return "back to the basics," connecting the importance of nutrition to health and cancer prevention. Similarly, women needed to be taught how to do self-exams. All four groups explained the community was in need of this type of educational campaign. Haitian and African-American women felt that younger women should learn about breast cancer and be instructed on how to properly perform self-exams much younger than 40 years of age. Their perspective was that waiting until 40 was too late. Women also explained that the silence in the community existed because cancer and illness were not commonly discussed in the community. They explained that more communication on billboards, in social groups, in churches, and in media was needed. Pastors, politicians, elders, cancer survivors and local celebrities were identified as the appropriate figures to be involved in these campaigns. No dissenting opinions were given to the perception that improved support in the community required more communication about cancer.

Yeah. We need to talk about it.

Interviewer: Okay, and doctors aren't talking about breast exams. Yes, not enough.

Interviewer: Now, this is a primary care doctor or specialty doctors?

Primary care.

Interviewer: Primary care. And then you said doctors tend to bring up the topic of breast cancer or mammograms after symptoms exist?

Yes. (Haitian woman)

Interviewer: And you also said more education is needed about how to do a breast self-exam. Right, yes.....All women in general so that it would help to catch a problem if there's a problem there. Interviewer: And then you talked about prevention requires changing the mindset. 
Yes, giving you knowledge to know the knowledge of understanding the

diagnosis. To understand what breast cancer is and how it occurs will help men and women to understand better so that they can know how to take care of themselves or treat themselves. (Haitian women)

Women wanted pastors, priests, clergy and other church leaders to improve their level of support that is disseminated to the congregation from the pulpit to encourage mammograms along with healthier lifestyles among Black women. African-American and Haitian women have called for more conversation about women's overall health and breast cancer screening specifically. Both feel that this leadership in the church could be more involved and directive about the health of their members.

We need to talk more about the disease. We need to talk more about it in our churches, in our neighborhoods, and among our family members.... Those who really know about it or have had the disease; so survivors, those who have survived.

Interviewer: And why are these places important? Why is the church important to do this discussion?

Because that's where, mostly, we are. (Haitian woman)

\section{Structural Impediments}

Although not related to culture, the participants discussed the structural impediments that impact their use of mammograms and other medical services. Both African-American and Haitian women identified these as barriers for women with and without health insurance coverage.

Burdensome process to qualify for economic support. For women who need financial support and go through the primary hospital that supports this community, the qualification process was characterized as challenging. Required paperwork was described as the most challenging part of the process. Relevant comments addressed the volume of paperwork that must be completed, the invasiveness of the questions that were 
asked, and the length of time that was needed to complete this process. African

American women addressed the length of time spent on-site to complete the process and Haitian women were most concerned with the invasiveness of the paperwork. Women from both groups commented that they wondered if the process was worth completing.

These barriers became more troublesome for a woman who felt that she had been disrespected in the course of communicating with hospital staff. Another woman felt betrayed by the insurance company when she was denied coverage after completing the mammogram but had accepted services with the understanding that the cost was covered. Ultimately, these events discouraged continued engagement by these women with the health care system and strengthened a perception already ingrained in many of the women that these exams were not important. These impediments further diminished the value of these health services.

So the first time I went this time around, which was earlier this year, the doctor wanted to see me again. They wanted to get a biopsy. But I never went for the biopsy because my insurance had canceled. But I had made another appointment to go this time around, and I told this [inaudible] At the end of the day, yeah, so I went again,...(African-American woman)

I started going to the 7th Avenue clinic because I heard through word of mouth, I got the information to go to them because I didn't have any health insurance, like this was like two years ago when I found out about it. So guess what, the first thing I did, I made my visit to go there. I made an appointment. I went down, waded through the process, everything that they had me do... (African American woman)

Like I was saying, when she said, that she goes to 711. To go to 711 , it's like pulling teeth! You have to give them all your life story and then you spend hours sitting there waiting. Who wants to do that?! Even if your health depends on it, you know. (Haitian woman)

Do you think that over here, they will refuse to treat you because someone does not have insurance or money? Do you think that this is what's happening over here? 
Yeah! They ask you what's your insurance, who's your carrier, and if you don't have one, they don't really care about you. (Segment completely in Creole). (Haitian women)

Limited accessibility. Perceived receptiveness by the women of their local clinics has been an important barrier for Haitian women. The women provided a series of comments regarding limited accessibility to the clinic. These comments addressed the regulations and processes in place to provide services to women who have limited or no insurance. They contend that the policies limit accessibility to services and deter poor women from seeking services even in cases of urgent need. These comments suggest that structural barriers instituted by the health care system significantly contributed to the health disparities of this community. Moreover, African-American and Haitian women were adversely affected by these policies because the majority of women depended on these clinics for care.

Even though they got these clinics that offers free Medicaid. Medicaid is a joke. (African-American woman)

That's what I said, they will give you some kind of treatment but huh...compared to Haiti, you go, even though, you're DYING on the floor, they will not give you anything unless, somebody with an open heart, we'll say okay, let me help you, even though you don't have the money. (Haitian woman)

Before it was not like that in the States. I used to go to the emergency room, with no insurance, and they will you know be more than happy to assist you, like I said they will bill you later but now it's a little bit stricter. You have to have a carrier. (Haitian woman)

... make it easy for anybody, with any, status you know, whether you're rich or poor. Make it accessible to everybody, because it it's accessible, most likely, I will get up and you know and go, but if it's not accessible, monetary wise you know, I'm not going to, I don't have \$800, to spend just on a mammogram. I mean, even if my health depended on it, you know. Even if your health depends on it, you don't have that money stashed there, and then you go and pay it, you know. And if you don't have a job, 
you know, you most likely won't have health insurance, or I mean, I tried to get health insurance for me and my daughter, it was going to cost us like $\$ 400$ a month. \$400! And I put you know, the lowest income level (laughs), I'm like, what is this? So we never got insurance. I mean now she has it through her job, I don't have, I don't have insurance. Then, you go to Medicare, it's another story (Haitian woman)

Do you think that over here, they will refuse to treat you because someone does not have insurance or money? Do you think that this is what's happening over here?

Yeah! They ask you what's your insurance, who's your carrier, and if you don't have one, they don't really care about you. (Segment completely in Creole). (Haitian women)

\section{Community's Recommendations to Improve Women's Participation}

Study participants made several recommendations to improve women's participation with mammograms and preventive care visits. Recommendations included interventions that addressed service delivery, gaps in education and training for community members, development of partnerships with local churches to improve health outcomes for congregation members, and training of service delivery personnel.

Recommendations have been summarized by listing the intervention, the activities that could be performed associated with that activity, the perceived problem that would be targeted by the intervention, and the rationale for the recommendation (Table 14). 


\begin{tabular}{|c|c|c|c|}
\hline \multicolumn{4}{|c|}{$\begin{array}{c}\text { Table 15: Participant Recommendations to Improve Community's Response to Breast Cancer } \\
\text { Screening }\end{array}$} \\
\hline Recommendation & Potential Activities & Target Problem & Rationale \\
\hline $\begin{array}{l}\text { Promote Breast Self- } \\
\text { Exams }\end{array}$ & $\begin{array}{l}\text { 1. Teach self-exams } \\
\text { 2. Discuss breast cancer } \\
\text { in churches } \\
\text { 3. Explain to women } \\
\text { about stages of } \\
\text { development for cancer } \\
\text { (transparency) } \\
\text { 4. Explain to women the } \\
\text { strengths and weaknesses } \\
\text { of self-exams (real deal) }\end{array}$ & $\begin{array}{l}\text { 1. Many women are } \\
\text { unaware of breast cancer } \\
\text { symptoms } \\
\text { 2. Some Haitian women } \\
\text { avoid mammograms } \\
\text { because they prefer } \\
\text { natural options. } \\
\text { 3. Some African } \\
\text { American women avoid } \\
\text { mammograms related to } \\
\text { trust }\end{array}$ & $\begin{array}{l}\text { Women who prefer } \\
\text { natural health care } \\
\text { options may be more } \\
\text { comfortable and willing } \\
\text { to engage in this activity. } \\
\text { Strongly recommended } \\
\text { by Haitian women for } \\
\text { Haitian women. African- } \\
\text { American women also } \\
\text { receptive to this idea. }\end{array}$ \\
\hline $\begin{array}{l}\text { Increase educational } \\
\text { efforts in community }\end{array}$ & $\begin{array}{l}\text { 1. Sponsor sister circles } \\
\text { 2. Develop an education } \\
\text { effort that targets seniors } \\
\text { 3. Use breast cancer } \\
\text { survivors to educate } \\
\text { women }\end{array}$ & $\begin{array}{l}\text { 1. Many women in the } \\
\text { community do not } \\
\text { understand the risks } \\
\text { presented by their } \\
\text { behaviors } \\
\text { 2. Misperception among } \\
\text { some Haitian women that } \\
\text { they are not at-risk for } \\
\text { developing breast }\end{array}$ & $\begin{array}{l}\text { 1. Education allows } \\
\text { women to better } \\
\text { understand their options } \\
\text { and empowers women to } \\
\text { attend to their health } \\
\text { 3. Opportunity to share } \\
\text { that disease is not a } \\
\text { death sentence } \\
\text { 4. Survivors are received } \\
\text { as credible } \\
\text { spokespersons for the } \\
\text { importance of screening }\end{array}$ \\
\hline $\begin{array}{l}\text { Incorporate pastors } \\
\text { for recruitment and } \\
\text { facilitation }\end{array}$ & $\begin{array}{l}\text { 1.Integrate health } \\
\text { promotion message and } \\
\text { behaviors in the pulpit } \\
\text { 2. Churches sponsor trips } \\
\text { to the clinics for exams } \\
\text { 3. Host routinely } \\
\text { scheduled screening } \\
\text { events in churches as an } \\
\text { incentive }\end{array}$ & $\begin{array}{l}\text { 1. African-American } \\
\text { women wanted more } \\
\text { leadership, coordination } \\
\text { of activities and explicit } \\
\text { commitment to women's } \\
\text { health } \\
\text { 2. Haitian women wanted } \\
\text { more guidance and } \\
\text { endorsement }\end{array}$ & $\begin{array}{l}\text { 1. Actions sponsor } \\
\text { commitment } \\
\text { 2. Actions sanction } \\
\text { preventive health } \\
\text { behaviors }\end{array}$ \\
\hline $\begin{array}{c}\text { Focus on } \\
\text { communication } \\
\text { about health-related } \\
\text { topics in the } \\
\text { community }\end{array}$ & $\begin{array}{l}\text { 1. Engage politicians and } \\
\text { media to be a part of the } \\
\text { dialog } \\
\text { 2. Create visible health } \\
\text { campaigns in the } \\
\text { community }\end{array}$ & $\begin{array}{l}\text { 1. African-Americans } \\
\text { wondered why local } \\
\text { politicians were not } \\
\text { engaged } \\
\text { 2. Both groups remain } \\
\text { silent about their health } \\
\text { and avoid disclosure } \\
\text { 3. Community is void of } \\
\text { billboards and other } \\
\text { health promotions } \\
\text { materials that would } \\
\text { educate women }\end{array}$ & $\begin{array}{l}\text { 1. Elevates as a } \\
\text { community-wide priority } \\
\text { 2. Promotes awareness } \\
\text { and health programming } \\
\text { activities } \\
\text { 3. Reduces the legacy of } \\
\text { silence in the community } \\
\text { about health issues }\end{array}$ \\
\hline
\end{tabular}




\begin{tabular}{|l|l|l|l|}
\hline \multicolumn{4}{|c|}{ Table 15: Participant Recommendations to Improve Community's Response to Breast Cancer } \\
Screening (cont.)
\end{tabular}

\section{Conclusion}

The content in chapter four was organized in response to six research questions that guided this study. The purpose of these questions was to explore the relationships of culture, ethnic identity, and social cohesion to the health perceptions and health behaviors of a bounded sample of African-American and Afro-Caribbean women. This study was set in a community challenged with a history of poorer health outcomes, residents who often find themselves disenfranchised from the broader county, and a community comprised of distinct ethnic and racial enclaves. Given this context, the researcher sought guidance from women who live in this community to understand how the three aforementioned concepts may significantly improve the proportion of women who complete mammography and see doctors for preventive medical care. These accounts offer insight into the continuation of high mortality rates, late diagnosis for breast cancer, and delayed testing for breast cancer in Broward County.

\section{Summary of Major Quantitative Findings from Phase 2}

Four hypotheses were tested in this study. The first hypothesis was the AfricanAmerican and Afro-Caribbean women do not have a statistically significant difference in 
their perceptions and health-seeking behaviors about cancer. This hypothesis was supported. Both populations have generally less favorable perceptions regarding their overall attitude about cancer, sense of urgency to screen, perceived risk, ability to complete a breast self-exam and perceived benefits in screening. There are some differences in terms of urgency with Afro-Caribbean women being higher than AfricanAmerican women but this is not significant.

The second hypothesis was that African-American and Afro-Caribbean women do not have a statistically significant difference in their health-seeking behaviors about breast cancer and other cancers. This was supported in terms of preventive medical visits with the proportion of women in both populations being very similar. However, in terms of screening for cancer this was not supported and African-American women were more likely to screen than Afro-Caribbean women.

The third hypothesis was that ethnic identity will not have a statistically significant influence on the health-seeking behaviors of African-American and AfroCaribbean women. This hypothesis was partially supported. If ethnic identity was measured only by ethnic membership or a nominal label, statistically significant differences were not found for preventive medical visits or for screening for cancer. However, defining ethnic identity by using either the MEIM total score or OGO increased the likelihood that identity would be significant and that differences were found for cancer screening.

The final hypothesis stated that ethnic identity will not have a statistically significant influence on the detection of social cohesion among African-American and Afro-Caribbean women. Interestingly, ethnic identity as defined by ethnic membership 
was the only measure of identity to have statistically significant differences for cohesion. Specifically, Afro-Caribbean women would have higher identity and lower social cohesion. Adding to the final model importance of identity to defining sense of self to investigate the presence of low and high cohesion found that time as a resident and educational attainment were negatively associated and importance of identity and use of a non-western provider were positively associated with higher cohesion.

There were no statistically significant differences in the percent of women who self-reported ever being screened for breast and cervical cancer and use of a primary care physician. Significant differences existed for women who have completed a PAP smear and who use a non-Western care provider. More Afro-Caribbean women use a nonwestern care provider and fewer Afro-Caribbean women have had a colonoscopy compared to African-American women. Although not significant, more Afro-Caribbean women self-report that they have never had a mammogram and more Caribbean women do not have a primary care doctor.

\section{Summary of Major Findings for the Qualitative Data from Phase 3}

Three major themes summarized the overarching views of the participants for why women were not engaged with the local medical systems. Women had conflicting views on the necessity of participating in preventive and cancer screening activities, some women understood the need for these services but refrained from care, women need more support to change their health behaviors, and structural challenges for the women within the local medical centers has alienated some women in the community or contributed to delaying care. 
The participants suggested three variables that may moderate a woman's choice to screen for breast cancer and participate in preventive care services. Moderators included: (1) fear related to the disease itself and fear about the social consequences that were anticipated to occur because they had disclosed their health status, (2) motivation, and (3) pride. Culturally attenuated factors for African-American and Afro-Caribbean women included: faith and religion as being prominent forces in both ethnicities and influenced their health behaviors. Differences, however, existed between African-American women and Haitian women in the level of influence faith had on personal health-seeking activities, as well as, the roles they expected clergy to embrace as they relate to supporting them to live a healthier lifestyle.

Health prevention within the context of the US health care system is a westernized model. Many women who have immigrated to this country have interacted with a health care system that contradicts their social and culture. Conflicting perceptions and expectations have contributed to Haitian and other Caribbean women avoiding or delaying their encounters with the US health care system.

Based on these findings, the final chapter will discuss the importance of this information to the overall landscape of existing research on breast cancer interventions and community engagement within communities with similar ethnic diversity to that of ZIP-code 33311. 


\section{CHAPTER V: DISCUSSION}

This final chapter briefly summarizes the preceding four chapters that include the problem, purpose of the study, and major research questions. This chapter then discusses the major findings from this research comparing these results to other published reports and commentaries. This chapter closes with recommendations that address improvements in public health practice, health disparities research, and outcomes for women of color.

\section{Summary of Study}

The present study explored whether ethnic identity and culture shaped perceptions about cancer and screening for breast cancer of a bounded sample of African-American and Afro-Caribbean women. It also explored the significance of these variables on women's health-seeking behaviors related to cancer screening and preventive medical visits. The conceptual framework was adapted from Carpiano's (2007) model of social capital. His model illustrated various pathways that lead to health outcomes within modern, urban communities. The research focused on the model's pathways and relationships of connectedness, values, cohesion, personal attributes, and health behaviors. Modifications of Carpiano's model were made to the constructs that represented values and connectedness and they were replaced by culture and ethnic identity, respectively.

Findings of Sussner and colleagues (2010) contributed to the use of nativity, country of origin, residency, acculturation, culture, and ethnicity as key independent variables. Ethnic identity was represented by the multi-ethnic identity measure (MEIM) and the other-group-orientation (OGO) scales to explore comparable outcomes to 
published reports. Methods included semi-structured discussion groups and a selfadministered survey to see if ethnic identity, culture, and social cohesion were associated with the health-seeking behaviors of African-American and Afro-Caribbean women. One hundred and twenty one women were recruited within and in the immediate vicinity of the targeted community for the purpose of gaining insight into unanswered questions. Complementary lines of inquiry were made using semi-structured group discussions and a cross-sectional survey to develop a fuller context for why Black women were not obtaining mammograms in a more timely fashion.

\section{Statement of the Problem}

Premature mortality rates among Black women remain an urgent public health issue. Black women have continued to have the highest premature mortality rates among all racial and ethnic groups, with Black women living in the Southern region of the US now having the highest rates in the nation (ACS, 2015). Such disparity presented as a critical issue for the South because Black women comprise over half of the Black population, with an estimated $53 \%$ of these women falling within the age group that should be receiving ongoing breast cancer exams. (US Census, 2015). The failure of Black women to adopt and maintain recommended breast screening behaviors is a complicated, multi-layered problem. Likewise, women's ability to access and use of screening resources have been influenced by broader constructs such as racism, poverty, culture, and faith (Musgove, Allen\& Allen, 2002; Brondolo, Gallo \& Myers, 2009; Danfort, 2013; Pater et al., 2014). To the extent that these circumstances have a similar effect on the health behaviors of African-American and Caribbean is unknown. 
The Breast and Cervical Cancer Early Detection Program (BCCEDP) has been a successful intervention nationally to provide financial support for mammograms and link Black and poor women to needed breast cancer related services. Despite the presence of BCCEDP in Broward County, the uptake of Black women to adhere to screening recommendations has plateaued and mortality rates have remained disproportionately higher for Black women who live in ZIP-code 33311 compared to Black women who live in other areas of Broward (State of Florida, 2015, Kobetz \& Parker, 2010). The fact that so many of the women had refrained or delayed with their breast exams despite the availability of BCCEDP suggested that this behavior was happening for reasons other than affordability. Other possible reasons included their perspectives toward cancer and cancer screening, most notably, their perceptions of risk for developing life-threatening disease.

\section{Purpose of the Study}

The purpose of this exploratory study was to develop an understanding as to why an ethnically diverse group of Black women who self-described as African American and Caribbean and lived within a bounded Broward County community were not complying with screening recommendations for breast cancer. Three aims were to explore women's perceptions and health-seeking behaviors toward cancer screening and preventive medical visits among a community of ethnically diverse Black women, determine if ethnic differences in their perceptions and health-seeking behaviors existed, and characterize the various community norms (as indicated by culture, ethnic identity, and social cohesion) that influenced the health-seeking behaviors of Black women in a lowincome neighborhood. 


\section{Discussion of Major Findings}

Differences in health-seeking behaviors existed between Afro-Caribbean and African-American women. More women, regardless of ethnicity, expressed a positive likelihood to screen for cancer and to complete a preventive medical visit in the future. However, in both cases, almost twice as many Afro-Caribbean women compared to AfroAmerican women indicated that they would not participate in a preventive medical visit or to screen for cancer. Low participation rates in both activities have contributed greatly to the high rates of mortality among Black women associated with breast cancer in Broward. Results suggested that engagement of Afro-Caribbean women in either activity would present the greater challenge for public health personnel and that screening for cancer remained an obstacle to improving the health status for some African-American women. Unlike most research conducted in the USA and abroad, ethnic variations were explored and detected. Jones et al. (2014) underscored the paucity of research that investigates ethnic variations in health perceptions, health behaviors, and health outcomes for Black women. Broward's women suggested similar strategies to those found by Jones et al. (2014) to improve early detection and diagnosis of breast cancer among Black women such as a need for education that offers women both skills and knowledge to address symptom recognition and interpretation of risk as well as capacity of programs to better support women's fears related to the consequences of cancer.

Data analyses in both studies have provided evidence that perceptions, needs, and education about breast cancer vary by ethnicity. Therefore, failure to document potential differences in receptiveness to interventions, perceptions of risk, or other critical ariables in public health may be missed opportunities to improve effectiveness of methodologies, 
validity of inferences drawn from research activities, and ultimately improve health outcomes. Continued work that explores heterogeneity in health perceptions, health behaviors, and participation in both preventive medical and cancer screening services would benefit women who live in Broward County.

\section{Ethnic Variations in Health Perceptions}

Mean rank scores indicated that there were no statistically significant differences between African-American and Afro-Caribbean women in their cancer-related health perceptions. Both populations expressed perceptions across all five RBDS scales that seemed less favorable toward engagement in screening for cancer and completing a mammogram. Less favorable RBDS scores suggest that women living in the target community have health perceptions that may predispose their non-adherence to recommendations commonly followed for breast screening. Similar studies also found that health perceptions did not differ among ethnically diverse subpopulations that represent Black women (Barbers \& Chiasson, 2006; Gwede, William, Thomas, et al., 2010). Gwede et al. (2010) found differences in terms of perceived risk and self-reported colonoscopies among Haitian, English-speaking Caribbean and African Americans in their Tampa-based study. These differences, while varied, were not significant and the perceptions were similarly less favorable (Gwede et al.). In both studies Black women could be characterized as having overall negative perceptions and attitudes that would discourage screening.

The close proximity of the two study sites may have resulted in respondents that share immigration histories and points of origin. Acculturation has not been associated with perceived worry for breast cancer among immigrant women who are Latina and of 
other ethnicities (April-Sanders, Oskar \& Shelton, 2016). However, evaluation findings of the national BCCEDP program have shown that targeted educational programs and collaborating with community-based organizations to implement these programs were effective strategies to reach poor and less acculturated women (Levano, Miller, Leonard, et al., 2014). Research on the relationships between health perceptions, health behaviors, and acculturation for ethnically diverse Black women is an emerging area of research and thus is not well understood. Particularly since length of time in residency was a significant variable in this study and for other studies that have investigated the effect of acculturation, future studies in this area are warranted.

\section{Health Perceptions and Health-Seeking Behaviors}

Of the five perceptions explored by the RBDS scales, perceived risk and perceived benefit may be the most vital indicators of the women's health-seeking behaviors. In terms of ethnicity, perceived risk exceeded other health perceptions measured by the RBDS scales and this finding was important because it aligned with prior evidence that established risk as a significant predictor of avoidance of cancer screening (Vernon et al., 1997; Vernon, 1999). However, Fair et al. (2012) showed evidence of an interaction between perceived risk and benefits that influenced the adherence of Black women to mammography recommendations. Their research indicated that lowest adherence occurs in situations where perceived risk was high and benefit low compared to Black women who perceived a lower risk for developing breast

cancer yet saw great benefit with screening. Thus, the comparison by ethnic membership supported their suggested interaction and a subsequent conclusion would be that AfroCaribbean women would have lower adherence to mammography because their 
perception of risk exceeded their perceived benefit. Ethnic membership alone therefore may not be the best concept of ethnicity to determine health behaviors of Black women. Using additional measures of ethnicity, such as MEIM, along with ethnic membership would clarify the level of influence that ethnicity offers to understanding health behaviors across diverse ethnic populations of Black women.

\section{Relationship of the MEIM to Women's Health Perceptions}

Ethnic identity represented by the OGO scale was related to ethnically diverse Black women's perceptions toward breast cancer and mammograms in Broward. This relationship was found for overall attitudes toward breast cancer, the sense of urgency about breast cancer, perceptions of risk, and perceived personal benefit in completing the exam. OGO accounts for a group's willingness to develop relationships and networks of members outside their ethnicity. Data suggests that characteristic is a protective factor for both African American and Afro-Caribbean women who live in Broward, particularly for women that are older $(60+$ years of age) and more established in the community. Additional analyses may show that OGO can serve as a protective factor for younger women (40-60 years of age) and Afro-Caribbean women who have recently moved to the community. Research conducted with similar populations found the OGO and other scales within the MEIM were protective factors related to genetic testing for breast and cervical cancers (Sussner et al., 2010).

Personal characteristics of women greatly influenced their attitudes and perspectives about screening for breast cancer. Educational attainment and age (60 years and older) were found to be protective characteristics of the women in Broward County. Zollinger, Champion, Monahan, et al. (2010) also found that higher education was 
associated with more positive attitudinal outcomes. In contrast, they found that older age was not protective but, in fact, encouraged higher levels of fatalism and reduced perceptions of risk. Differences between the two studies could be explained by variations in the educational levels of the two samples. Fair et al. (2013) found that educational attainment was inversely related to where Black women fell along among four risk/benefit categories and it, in turn, influenced fatalism. Measures in the other group orientation scale along with the characteristics identified by the study participants in the discussion groups related to their culture and social environments were important to their perceptions about mammography. Differences in dispositions about these variables would result in differences in women's attitudes. More research is needed to better understand the influence of OGO and other characteristics identified in this study on perceptions of women about breast cancer and mammography.

Measures of identity (OGO score) and social cohesion (nativity, parental nativity, and years as a resident within the community) were significantly associated with women's health perceptions. In Broward stronger OGO scores were associated with women who were more favorable to cancer screening testing and had more favorable perceptions about cancer. Women who were more engaged with people of other ethnicities would also have perceptions more aligned with screening for cancer. Mean distribution of OGO by ethnicity found that African-American women had more involvement than Afro-Caribbean women and were more likely demonstrate the perceptions more aligned with a willingness to screen for cancer. Moreover, OGO was the only MEIM measure found to have a significant association with African-American and Afro-Caribbean women's health perceptions, unlike the Sussner (2010) study where 
several measures from the MEIM and other identity scales were found to have a significant association with the health perceptions of ethnically diverse Black women living in New York.

OGO may also prove to be a valuable tool to detect absence or presence of cohesion both within social and across social networks. Since the OGO scale references the likelihood and potential of members of one group to work with other groups, future investigations may find the OGO as an appropriate proxy for social cohesion.

\section{Explanation for Behaviors of Late-Diagnoses and Non-Adherence}

Three themes were established to organize the ideas offered by women to explain why they were not adhering to screening recommendations. The themes were conflicting views on the necessity of medical services, social support, and structural impediments. These views were derived from the culturally moderated perceptions of women who lived in the target area about their health. These views also described factors that they perceived directly influenced their health-seeking activities. While the women did not

provide responses that addressed the relationship of social cohesion to health-seeking behaviors, many comments were relevant to the influence of culture and ethnic identity.

The first theme pertained to a normative behavior of the community where many women generally refrained from seeing a doctor, for any reason, unless a person has experienced symptoms that indicate illness (i.e., pain, bleeding, etc.). By illness, the person had to be extremely affected by the illness before taking action,

Once a symptom occurs most women will go to a medical provider. Women might go to the local clinic, visit their private doctor, seek relief from a non-traditional healer, self-medicate with herbs and other traditionally endorsed remedies, or ignore the 
symptoms and carry on. It is at this point where the second theme, understand the importance of prevention but choose to refrain from care, becomes very powerful. Women enter a crossroads where they have to "prioritize" themselves and their health. Roman, Meghea, Ford, et al. (2014) confirmed the sentiments of the women in this community that competing priorities and responsibilities to others often take precedence and force personal care to come second or third to these overriding issues. Study participants, both African-American and Afro-Caribbean discussed that women in this community, and Black women in general, habitually neglected their personal health. In fact, many women in this community discussed the immediacy of managing other comorbidities, finding gainful employment, supporting extended family or neighbors, and serving as caretaker for other family members who may live in Florida or in their native countries, as examples of their real-life issues that prevent women from finding time to deal with what they see as "yet another issue" over making their personal health a priority.

Hall, Rim, Johnson-Turbes, Vanderpool and Kamalu (2012) evaluated the African-American Women Mass Media Campaign and their findings mirrored those of this study. Women from the Hall et al. study also participated in focus groups where they shared that women understood the importance of mammograms but the competition of other priorities often delayed uptake. Beyond the prioritization of personal health were two mediating factors that also influenced visiting a doctor or completing a mammogram. During the discussion groups women described fear and motivation as mediating factors that influenced health-seeking behaviors.

Fear 
Fear corresponds to anxiety about the disease and to the possible social consequences associated with discovery and disclosure of having cancer to others. [Major Medical Mystery: Why People Avoid Doctors (2000, October 31). (Clipping from electronic reprint by the NY Times). Electronic copy in possession of author.]. Women in this study described another aspect of fear that focused on the fear of anticipated physical injury and bruising from the exam process while other women described their fear of failing to manage unknown costs incurred from the exam. The second set of perspectives about fear expressed in this study was reinforced by similar comments in the BCCEDP program evaluation (Hall et al., 2012) that pertained to women's sense of fear. "Among the common causes of fear related to breast cancer were the physical pain of being sick, hair loss, loss of finances, inability to work or pay for treatment, and longterm treatment of the disease" (Hall et al., 2012, p.5). Both perspectives of fear influenced a woman's choice to postpone engagement with medical services and added to the complexity of avoiding preventive services.

\section{Motivation}

"Self-love" and "self-respect" were terms used by the women to characterize what motivated them to seek medical care. For African-American women their self-love was directed at their responsibility to serve as a caregiver for another person. They suggested that there was a direct relationship between motivation and the strength of their self-love whereby an increase in strength led to increased motivation, which in turn, led to commitment and a sense of importance about their health status. Haitian women also talked about factors that motivated them to stay healthy, but their source of motivation was directed by their perceived pride associated with their common history of having 
regional prominence as Haitians. The honor that they perceived as "Haitian people" was built upon a perceived expectation of their elders, members of the social network, and other Caribbean people to live up to an honor bestowed upon them from their past. Jones and Paxton (2015) examined the relationship of motivation, structural barriers, support, and physical activity among breast cancer survivors. They found that lack of motivation was the primary factor associated with women failing to participate in physical activity.

\section{Structural Impediments}

\section{Ability to Pay for Services}

The ability to pay for a medical procedure may not be the most influential factor for women of African descent to complete medical services in today's communities. Participants' responses on the cross-sectional survey and comments made in the discussion groups indicated that ability to pay for medical services in this community challenged women to complete these exams, but for a significant number of women with resources to cover the associated costs (insurance), they would still refrain from completing a preventive medical visit $(10 \%)$ and screen for cancer (16\%). Similarly, Jones et al. (2014) found that delay in testing among ethnically diverse black women was attributed to non-financial factors that included: fear of detecting breast abnormality, fear of cancer treatments, fear of partner abandonment, embarrassment disclosing symptoms to healthcare professionals, taboo, and stigmatism. Life stressors such as perceived gender and racial discrimination have been associated with reduced breast screening (Dailey, Kasl \& Jones; 2008; Jacobs et al., 2014). Interestingly, gender discrimination was found to reduce mammography compliance among Black women who were insured and more educated because they were significantly less likely to have reported high 
perceived control over recovering from cancer (if diagnosed), less likely to believe that mammography screening was very useful in detecting breast cancer, and more likely to report they were somewhat likely to develop breast cancer, more likely to report a stressful life event, and report being disrespected by the technologists (Daily, Kasl \& Jones, 2008).

The ability to pay for services cannot be dismissed, however, as an important factor because, for many women in this community, if they were insured they would utilize medical services. Not having insurance contributed to a third variation of fear that many women described in this study - the inability to cover costs. For example, one Haitian woman explained that she delayed services because of the anticipated cost of the exam while another Haitian woman was reluctant to pursue additional services because of fear of the debt she would incur and her inability to cover these costs. The literature also confirms that socioeconomic factors including income and insurance were positively associated with mammography (Deshpande et al., 2009). Therefore, the availability of programs such as BCCEDP has the opportunity to play a vital role in this community. Most women (61.1\%) in this study were not aware that BCCEDP provided support for mammograms. Of the 77 women who were aware of their services, only $38.1 \%$ had used their services. More awareness and promotion about the availability of assistance with breast and cervical assessments through the BCCEDP program is warranted to improve breast cancer screening among Black women in Broward County.

\section{Trust}

Trust was an important concept for African-American and Haitian women.

African-American women discussed trust in the context of not trusting and consistently 
finding themselves questioning the medical system and the reliability of health information given to the community about diseases and their health behaviors. AfricanAmerican women also mentioned the legacy of the Tuskegee experiment and its continued influence to limit their involvement with the medical process. This skepticism was enhanced by the perceived frequency that contradictory content has been released into the public domain. The frequency with which directives can change has resulted in women lowering their trust and belief in health information.

Haitian women had mixed reactions related to trust in the health messages that they were exposed to about risk of developing breast cancer. A few of the women questioned the idea of vulnerability to developing cancer and other health conditions. Prior to coming to this country, Haitian women explained that they had minimal exposure to conversations about breast cancer and other diseases. They also could not remember being exposed to information about screening for breast and cervical cancer nor exposure to literature about preventing these diseases. These circumstances have resulted in some Haitian women being cautious and hesitant toward embracing the US health-care system.

In one discussion group a Jamaican woman questioned whether diseases among other Jamaicans living in the US happened because of their exposure to the US. Then a number of Haitian women explained that Americans and Haitians had the same vulnerabilities to diseases, but their culture made it acceptable not to talk about these events. According to participants, both the health-care systems and community members do not openly talk about health issues, risks for diseases, and prevention of disease due to cultural beliefs and norms. It is unclear from the conversations, however, how much outreach, education, and health promotion activities occurred from the public health and 
medical sectors in Haiti. If there has been limited outreach into the communities, their absence may have elevated the legitimacy and authority of non-traditional practices and these perceptions would naturally continue among Haitian and other Caribbean women who have immigrated to ZIP-code 33311 and environs.

Haitian women explained that their culture remained silent about health issues, particularly those illnesses that have been associated with high mortality (i.e., HIV and cancer). This perpetual silence about breast cancer adds to a continued misconception about their susceptibility to developing breast cancer and a belief that breast cancer is not a problem within their families. Moreover, these perceptions only lead to delay and avoidance of mammograms and other health services. Gierisch and colleagues (2009) found that lack of knowledge and not thinking about mammograms are needed was one of the four barrier categories associated with non-adherence to mammography. As indicated in all of the discussion groups, more communication that is transparent and straightforward is needed to improve trust and empower poor and less-educated women to participate in the medical system. O’Malley, Sheppard, Schwartz and Mandelblatt (2004) found that for low-income African-American women, primary care characteristics were more predictive of patient trust than patient characteristics. Also, greater overall trust in their primary care provider was associated with an increased use of preventive services.

\section{Women Need Support}

Both African-American and Afro-Caribbean women described the importance of a support network to initiate and remain engaged in the health care system.

Recommendations from the women were to include clergy, cancer survivors, and the 
broader community in this effort. Kissal, Vural, Ersin, Solmaz (2017) found that social support counteracts fear and promotes completion of mammograms. Holt, Lee, and Wright (2008) also found that a spiritually based approach to breast cancer awareness may be more effective than the secular approach, because it caused women to more actively process the message, and in doing so, women were persuaded by the content of the message. Women from both populations indicated that the lack of support from their social networks, family, and the community where they live has discouraged their involvement with mammograms. Sadler et al. (2007) underscored the importance of a support system whereby the perception of support from social networks established local churches facilitated Black women living in Los Angeles to complete mammography and enabled them to become healthier overall. As such, women in Broward wanted to see more direction and involvement of the clergy from the pulpit to support women's breast health specifically and women's health overall. African-American women would like pastors to coordinate and participate in church-sponsored events while Haitian women felt that education and screening activities should be sanctioned by the pastor so that women would feel more comfortable with the activity.

African-American and Afro-Caribbean women suggested that absence of communication and support from family, social networks, and the broader community would cause the uptake of mammography in Broward to remain low. Many of the African-American participants called attention to factors such as the absence of billboards and literature in the community about breast cancer and other cancers in the community, limited events where health concerns were discussed in public forums, and on local radio stations. Absence of health issues in the past political campaign platforms 
from local candidates was seen as evidence that women's health status was not a priority in their community nor among their leadership. Haitian women and African-American women suggested that pastors and other faith leaders should become more involved from the pulpit by delivering messages to better support women's health-seeking behaviors.

The importance of social support for Black women in Broward reflects a cultural characteristic of Black women nationally. Successful BCCEDP grantees such as the Dallas Cancer Disparities Community Research Coalition improved women's adherence to screening recommendations by implementing a broad-based support network similar to that suggested for Broward to address the disproportionate number of women being first diagnosed with breast cancer after progression to Stage III and Stage IV (Cadarelli et al., 2011). In Boston comparable efforts were used to develop a case management intervention to address the uptake of mammograms among Black women (Clark et al., 2009). Collaboration with a visible and comprehensive community coalition is a reasonable approach to address health disparities in Broward.

For a segment of women in Broward support becomes critical at the point that a diagnosis of cancer has been given versus support to screen. These women understood both the risks of avoidance and the benefits that come from completing a mammogram, but remain non-compliant because of anticipated apprehensions associated with a positive diagnosis. Concerns were with assurance that adequate support existed to move past a diagnosis, the necessary steps to take to move on, and for some women, detection of cancer continued to be perceived as a death sentence. For some women it was easier to manage their situation by delaying or avoiding mammograms. 


\section{Recommendations}

\section{Recommendations for Practice}

More education in the community is needed regarding breast cancer, prevention, testing methods and treatment options. Many of the women spoke candidly about the lack of knowledge about breast cancer, uncertainty about how to examine their own breasts for cancer, and an uncertainty, to some extent, of how to talk with other women and men about creating support in their community for women's health services. Broward BCCEDP offered 98 educational programs at health fairs or other presentations where they completed outreach to 7,657 people. [T.M. /Private communication through unpublished report]. While these educational events were convened in ZIP-code 33311, the content of these events varied and many were not educational but opportunities to disseminate information in public venues versus conducting a planned session that covered a PowerPoint and planned demonstration. . The Center for Disease Control and Prevention has developed a Guide to Community Preventive Services that contains recommendations for evidence-based interventions that are effective strategies to increase breast and cervical cancer screening (Levano et al., 2014). Staff would benefit from accessing this resource and reviewing the various strategies implemented nationally by grantees to deliver educational activities and to foster collaborations in communities for the purposes of sustainability and saturation within targeted locations. Best practices could be tailored to address the context of the issues, goals, and populations within Broward County to improve health outcomes among Black women.

BCCEDP staff were challenged in reaching the community because of poorly attended community-sponsored health events, lack of available funds to invest in 
advertising activities within the agency, and few staff available to conduct outreach activities. Given the barriers faced by BCCEDP to conduct educational sessions, resources should prioritize activities that contain information that is direct, transparent; the content should be framed by risk messages and survival messages. Both populations also suggested that survivors would be the best qualified and most welcomed to provide this training, education, and support. Active involvement of survivors during the educational sessions enhances the credibility of the spokesperson and promotes hope related to surviving breast cancer, if detected.

Incorporate pastors from the local churches to take an active role with recruitment and support of women in the clergy to engage in ongoing clinical services for their breasts. Their role will include speaking consistently from the pulpit regarding the importance of screening and establishing a relationship with a doctor. Pastors can also capitalize on their position by sponsoring health events where women are brought from the church to clinics for testing. Since the presentation of preliminary data to the project's community advisory board BCCEDP staff have implemented a targeted plan to collaborate with faith institutions to create tailored educational activities that are delivered within church settings. This has become and ongoing priority within the program.

Establish a community-based educational campaign where a community advisory board is involved in the planning, implementation, and management of the activities. The advisory board includes representatives from all segments of the community who are invited to participate as community stakeholders. Their charge is to integrate healthier lifestyles into the framework of the neighborhood while promoting cultural understanding 
and respect for all segments of the community. The board should encourage activities to build upon the social capital that exists in the neighborhood and seek additional resources to expand their capacity to support initiatives that address healthier experiences for residents throughout their lifespan. A full list of recommendations to improve health conditions in ZIP-code 33311 along with data sources has been arranged as Figure 8. 


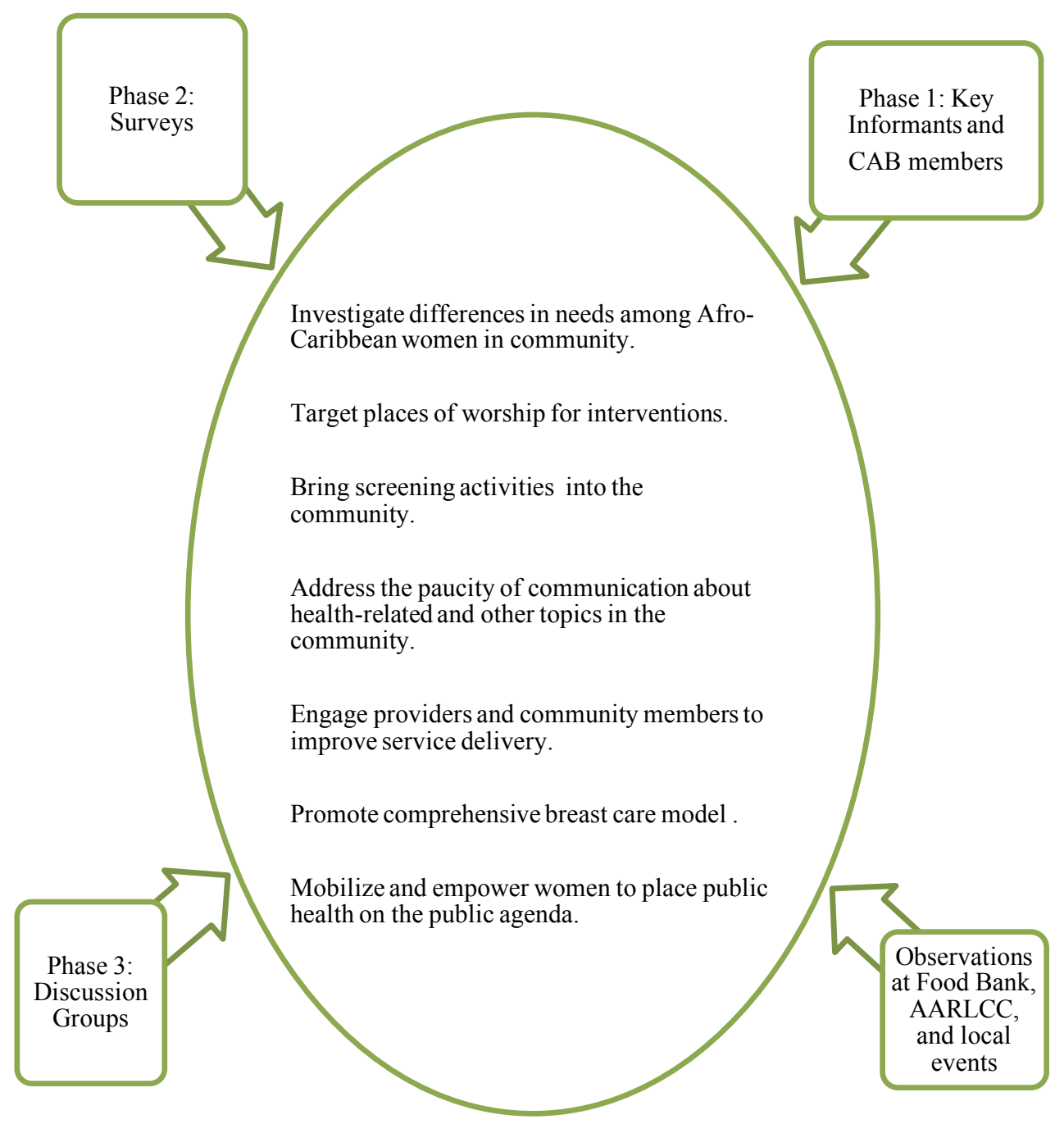

Figure 8: Future Actions to Engage Public Health Personnel in the Community 


\section{Recommendations for Improving the Research}

\section{Study design}

This cross-sectional study had several limitations. First, as a cross-sectional study, the design restricts the scope from which inferences can be drawn about the findings and causality cannot be determined from the data. Second, response burden was a major concern in developing the survey. The instrument was piloted and changes were made to that responded to observations and feedback during the pilot to address issues of response burden, clarity, and comprehension.

A primary interest in this study was to explore the influence of ethnic identity on health behaviors and perceptions and compare results with a study conducted in New York that had a sample similarly diverse in ethnicity. The researcher was unable to compare the two samples as exactly as planned.

\section{Instrumentation}

The instrument would have been more useful if it contained items that allowed for more precision in recording timing of screening and services among the participants. Questions about screening asked about past participation as "ever" (indiscriminate time in the past). Behaviors should have also included one or two finite periods of time (i.e., in the past 2 year) along with the ever option. Adding this line of questioning would have added meaning to questions related to adherence to screening recommendations and use of BCCEDP services.

Qualitative questions were piloted along with the cross-sectional survey. During pilot testing sessions, length of the questions and transitions appeared to work. However, during the full implementation of the study the delivery was more accelerated to get all of 
the items asked during the allotted one hour. This researcher would consider reducing the instrument to 3 - 4 lead questions and then allow the flow of the conversation to direct the remainder content of the conversation and close with a standard question as was done for this study.

\section{Implementation of the Study}

Changes were made to the methodology regarding assignment of participants to the discussion groups. The original intent was to stratify participants by ethnicity then assign them to a discussion topic. Recruitment and enrollment was more difficult and took longer than anticipated. Enrollment did not pick up until mid-November and this placed the study behind two months. Even when enrollment began to pick up, the number of people who self-described as Caribbean were few. Coordinating Caribbeanonly discussion groups were more challenging than the African-American groups. Challenges occurred in identifying eligible and willing Caribbean adults, enrolling them into the study, and then coordinating a time that accommodated everyone. After counsel with the dissertation chair, a decision was made to merge ethnicities if needed. Therefore three Caribbean sessions were completed in this study. Sessions were coordinated as ethnically mixed (African-American and Afro-Caribbean), Haitian (English or Creole language), and African American only.

\section{Recommendations for Future Research}

Two questions, length of stay as a resident of the community and length of stay as a resident of the county, had responses that were dichotomized for analysis as either more than 10 years or less than 10 years. This decision may have lowered the reliability of the measures used in this study. For example, the complexities of using variables such as 
nativity have a relationship to things such as their health perceptions, behaviors, and

income that vary over the increments of time. Future analysis should look more closely at the measures of residency and reassess these analyses using various intervals of time instead of a dichotomized measure.

\section{LIST OF REFERENCES}

American Cancer Society. Breast Cancer Facts \& Figures 2015-2016. Atlanta: American Cancer Society, Inc., 2015.

April-Sanders, A., Oskar, S., Shelton, R. C., ...\& Tehranifar, P. (2027). Predictors of Breast Cancer Worry in a Hispanic and Predominantly Immigrant Mammography Screening Population. Women's Health Issues, 0(0). [Epub Ahead of Print] doi: 10.1016/j.whi.2016.10.003

Brondolo, E., Gallo, L. C., \& Myers, H. F. (2009). Race, racism and health: disparities, mechanisms, and interventions. Journal of Behavioral Medicine, 32(1), 1-8. http://doi:10.1007/s10865-008-9190-3

Cardarelli, K., Jackson, R., Martin, M., Linnear, K., Lopez, R., Senteio, C., ... Johnson, E. S. (2011). Community-Based Participatory Approach to Reduce Breast Cancer Disparities in South Dallas. Progress in Community Health Partnerships : Research, Education, and Action, 5(4), 375-385.

Carpiano, R.M. (2007). Neighborhood social capital and adult health: An empirical test of a Bourdieu-based model. Health \& Place, 13(3), 639-655.

Clark, C.R., Baril, N., Kunicki, ..., et. al. (2009). REACH 2010 Breast and Cervical Cancer Coalition. Addressing social determinants of health to improve access to early breast cancer detection: results of the Boston REACH 2010 Breast and Cervical Cancer Coalition Women's Health Demonstration Project. Journal of Women's Health (Larchmt), 18(5), 677-90. http://doi: 10.1089/jwh.2008.0972.

Dailey, A. B., Kasl, S. V., \& Jones, B. A. (2008). Does Gender Discrimination Impact Regular Mammography Screening? Findings from the Race Differences in Screening Mammography Study. Journal of Women's Health (2002), 17(2), 195206. http://doi.org/10.1089/jwh.2006.0257

Danforth, D. N. (2013). Disparities in breast cancer outcomes between Caucasian and African American women: a model for describing the relationship of biological and nonbiological factors. Breast Cancer Research : BCR, 15(3), 208. http://doi.org/10.1186/bcr3429 
DeSantis, C. E., Fedewa, S. A., Goding Sauer, A., Kramer, J. L., Smith, R. A. \& Jemal, A. (2016). Breast Cancer Statistics, 2015: Convergence of Incidence Rates Between Black and White Women. Cancer: A Cancer Journal for Clinicians, 66 (1), 3142. doi: $10.3322 /$ caac. 21320

DeSantis, C. E., Lin,C. C., Mariotto, A. B., Siegel, R. L., Stein, K. D., Kramer, J. L., ... \& Jemal, A. (2014). Cancer Treatment and Survivorship Statistics, 2014. Cancer: A Cancer Journal for Clinicians, 64 (4), 252-271. doi: 10.3322/caac.21235

Kobetz, E. \& Parker, D. Cancer Data for South Florida: A Tool for Identifying Communities in Need, Report to the Health Foundation of South Florida. Disparities and Community Outreach Core, University of Miami Sylvester Comprehensive Cancer Center; 2010. Research Funded by Health Foundation of South Florida and community-based organizations.

Garbers, S. \& Chiasson, M. A. (2006). Breast Cancer Screening and Health Behaviors among African American and Caribbean Women in New York City. Journal of Health Care for the Poor and Underserved 17(1), 37-46. The Johns Hopkins University Press. Retrieved February 18, 2017, from Project MUSE database.

Gierisch, J.M., O’ Neill, S. C., Rimer, B. K., DeFrank, J. T., Bowling, M. \& Skinner, C. S. (2009). Factors associated with annual-interval mammography for women in their 40s. Cancer Epidemiology, 33(1), 72-78. http://

doi.org/10.1016/j.cdp.2009.03.001

Gullatte, M. (2006). The influence of spirituality and religiosity on breast cancer screening delay in african american women: Application of the theory of reasoned action and planned behavior (TRA/TPB). ABNF Journal, 17(2), 89-94. Retrieved from http://ezproxy.fiu.edu/login?url=http://search.proquest.com.ezproxy.fiu.edu/docvie w/218864531?accountid=10901

Gullatte, M.M., Brawley, O., Kinney, A. et al. (2010). Religiosity, spirituality, and cancer fatalism beliefs on delay in breast cancer diagnosis in African American Women. Journal of Religious Health, 49(1), 62-72. http://doi:10.1007/s10943008-9232-8

Gwede, C. K., William, C. M., Thomas, K. B., Tarver, W. L., Quinn, G. P., Vadaparampil, S. T., ... Meade, C. D. (2010). Exploring Disparities and Variability in Perceptions and SelfReported Colorectal Cancer Screening Among Three Ethnic Subgroups of U.S. Blacks. Oncology Nursing Forum, 37(5), 581-591. http://doi.org/10.1188/10.ONF.581-591

Hall, I. J., Rim, S. H., Johnson-Turbes, C. A., Vanderpool, R., \& Kamalu, N. N. (2012). The African American Women and Mass Media Campaign: A CDC Breast Cancer 
Screening Project. Journal of Women's Health, 21(11), 1107-1113. http://doi:10.1089/jwh.2012.3903

Holt, C.L., Lee, C., Wright, K. (2008). A spiritually based approach to breast cancer awareness: cognitive response analysis of communication effectiveness. Health Communication, 23(1), 13-22. http://doi: 10.1080/10410230701626919.

Jacobs, E. A., Rathouz, P. J., Karavolos, K., Everson-Rose, S. A., Janssen, I., Kravitz, H. M., ... Powell, L. H. (2014). Perceived Discrimination Is Associated with Reduced Breast and Cervical Cancer Screening: The Study of Women's Health Across the Nation (SWAN). Journal of Women's Health, 23(2), 138-145. http://doi.org/10.1089/jwh.2013.4328

Jones, A., \& Paxton, R. J. (2015). Neighborhood disadvantage, physical activity barriers, and physical activity among African American breast cancer survivors. Preventive Medicine Reports, 2, 622-627. http://doi.org/10.1016/j.pmedr.2015.07.010

Jones, C. E., Maben, J., Jack, R. H., Davies, E. A., Forbes, L. J., Lucas, G., \& Ream, E. (2014). A systematic review of barriers to early presentation and diagnosis with breast cancer among black women. BMJ Open, 4(2), e004076. http://doi.org/10.1136/bmjopen-2013-004076

Kissal, A., Vural, B., Ersin, F. \& Solmaz, S. (2017).The effect of women's breast cancer fear and social support perceptions on the process of participating in screening, Health Promotion, 1, 757-759. http://doi: 10.1177/1757975916677174. [Epub ahead of print]

Levano, W., Miller, J. W., Leonard, B., Bellick, L., Crane, B. E., Kennedy, S. K., ... Tharpe, F. S. (2014). Public Education and Targeted Outreach to Underserved Women Through the National Breast and Cervical Cancer Early Detection Program. Cancer, 120(0 16), 2591-2596. http://doi.org/10.1002/cncr.28819

Leyva, B., Nguyen, A.B., Allen, J.D. et al.(2015). Is Religiosity associated with cancer screening? Results from a national survey.`Journal of Religion and Health, 54, 998-1013. doi:10.1007/s10943-014-9843-1

Livaudais-Toman, J., Karliner, L. S., Tice, J. A., Kerlikowske, K., Gregorich, S., PérezStable, E. J., ... Kaplan, C. P. (2015). Impact of a primary care based intervention on breast cancer knowledge, risk perception and concern: A randomized, controlled trial. Breast (Edinburgh, Scotland), 24(6), 758-766.

http://doi.org/10.1016/j.breast.2015.09.009

Moore S, Stewart S, Teixeira A. (2014). Decomposing social capital inequalities in health. Journal of Epidemiology and Community Health, 68, 233-238. 
Musgrave, C. F., Allen, C. E., \& Allen, G. J. (2002). Spirituality and health for women of color. American Journal of Public Health, 92(4), 557-60. PMCID: PMC1447116

Mandelblatt, J., Mandelblatt, S. , Gold, S. ..., et al. (1999). Breast and Cervix Cancer Screening among Multiethnic Women: Role of Age, Health, and Source of Care. Preventive Medicine, 28, 418-425. http://www.idealibrary.com on

Orom, H., Kiviniemi, M. T., Shavers, V. L., Ross, L., \& Underwood, W. (2013). Perceived risk for breast cancer and its relationship to mammography in Blacks, Hispanics, and Whites. Journal of Behavioral Medicine, 36(5), 466-476. http://doi.org/10.1007/s10865-012-9443-z

Patel, K., Kanu, M., Liu, J., Bond, B., Brown, E., Williams, E., ... Hargreaves, M. (2014). Factors influencing Breast Cancer Screening in Low-Income African Americans in Tennessee. Journal of Community Health, 39(5), 943-950. http://doi.org/10.1007/s10900-014-9834-x

Roman, L., Meghea, C., Ford, S., Penner, L., Hamade, H., Estes, T., \& Williams, K. P. (2014). Individual, Provider, and System Risk Factors for Breast and Cervical Cancer Screening Among Underserved Black, Latina, and Arab Women. Journal of Women's Health, 23(1), 57-64. http://doi.org/10.1089/jwh.2013.4397

Sadler, G. R., Ko, C. M., Cohn, J. A., White, M., Weldon, R., \& Wu, P. (2007). Breast cancer knowledge, attitudes, and screening behaviors among African American women: the Black cosmetologists promoting health program. BMC Public Health, 7, 57. http://doi.org/10.1186/1471-2458-7-57

Smith-Bindman, R., Miglioretti, D.L., Lurie, N., Abraham, L., Barbash, R.B., Strzelczyk, J., et al. (2006). Does Utilization of Screening Mammography Explain Racial and Ethnic Differences in Breast Cancer? Annals of Internal Medicine, 144, 541-553. http://doi: 10.7326/0003-4819-144-8-200604180-00004

Sussner, W.M., Edwards, T.A., Thompson, H.S., et al. (2011). Ethnic, racial and cultural identity and perceived benefits and barriers related to genetic testing for breast cancer among at-risk women of African descent in New York City. Public Health Genomics, 14, 356-370.

Vernon, S.W. (1999). Risk perception and risk communication for cancer screening behaviors: A review, Journal of the National Cancer Institute, 1999, 101-119. 21.

Vernon, S. W., Myers, R. E., \& Tilley, B. C. (1997). Development and validation of an instrument to measure factors related to colorectal cancer screening adherence. Cancer Epidemiology Biomarkers, 6, 825-832. 
US Census, 2014. 2010-2014 American Community Survey 5-Year Estimates. Selected characteristics of the native and foreign born populations for broward county. U.S. Census Bureau, 2010-2014 American Community Survey 5-Year Estimates.

Retrieved from

http://factfinder.census.gov/faces/tableservices/jsf/pages/productview.xhtml?src=C $\mathrm{F}$

Zollinger, T. W., Champion, V. L., Monahan, P. O., Steele-Moses, S. K., Ziner, K. W., Zhao, Q., ... Russell, K. M. (2010). Effects of Personal Characteristics on AfricanAmerican Women's Beliefs about Breast Cancer. American Journal of Health

Promotion : AJHP, 24(6), 371-377. http://doi.org/10.4278/ajhp.07031727 
VITAE

\section{SHEILA MCKINNEY}

Born, Nashville, Tennessee

1984-1988

B.A., Political Science

Northwestern University

Evanston, Illinois

1994-1999

M.A., Educational Administration

The Catholic University of America

Washington, District of Columbia

2010-2017

Doctoral Candidate

Florida International University

Miami, Florida

Graduate Assistant

Florida International University

Miami, Florida

\section{PUBLICATIONS AND PRESENTATIONS}

McKinney, S. \& Palmer, R. C., (2014). The influence of gender on colorectal cancer knowledge, screening intention, perceived risk and worry among African Americans in south Florida. Journal of Community Health, 39:230-238.

Madhivanan P., Barreto, G.A., Revawala, A., Anderson, C., McKinney, S. \& PierreVictor, D. (2013). Where are we with partner treatment in bacterial vaginosis? A critical appraisal of the latest systematic review. Sexually Transmitted Disease, 40(6):518.

Palmer, R.C. \& McKinney, S. (2011). Health care provider tobacco cessation counseling among current African American tobacco users. Journal of the National Medical Association, 103(8):660-667.

Palmer, R.C., Chhabra, D. \& McKinney, S. (2011). Colorectal cancer screening adherence in African-American men and women 50 years of age and older living in Maryland. Journal Community Health, 36(4):517-524.

McKinney, S. , McCoy, H. V. \& Rubin, M. Exploring HIV Testing in Miami-Dade County Florida: HIV Testing Knowledge, Stigma, and HIV/AIDS Messages, Robert Stempel College of Public Health and Social Work, Florida International University, July 23, 2015. Commissioned by the Florida Department of Health in Miami-Dade County. 
McKinney, S., Shaw-Ridley, M. \& Darrow, W. (2017). Ethnic History, Faith, and Food: Cultural Norms Selected to Promote Breast Cancer Screening Among Women of African Descent. Dissertation presented at Society for Public Health Education 68th Annual Meeting Scaling New Heights: Health for All, Denver, CO, March 30-April 1, 2017.

Mukherjee, S., McKinney, S. \& Darrow, W. AIDS and gay-related stigma among students attending a large Hispanic-serving university. Paper presented at the American Public Health Association 144th Annual Meeting and Expo, Oct. 29 - Nov. 2, 2016, Denver, $\mathrm{CO}$.

McKinney, S. \& Shaw-Ridley, M. "I thought you'd never ask.”: Community conversations about breast health with ethnically diverse Black women in South Florida. Poster presented at the $14^{\text {th }}$ Disparities in Health in America: Working Towards Social Justice, June 20-25, 2016. Houston, TX.

Palmer, R. C. \& McKinney, S. Does message frame influence colorectal screening intentions among African Americans? Poster presented at the American Public Health Association 142nd Annual Meeting and Expo, November 15-19, 2014, New Orleans, LA.

McKinney, S., Palmer R. C. \& Anderson, C. Gender differences in intention to screen for colorectal cancer among African American community health center patients. Poster presented at the American Public Health Association 141st Annual Meeting and Expo, November 2-6, 2013, Boston, MA.

Felizzola, J., Pernia, E. \& McKinney, S. Latino Southern REACH Project: Lessons learned from community based participatory research (CBPR) to assess HIV/AIDS needs assessment within human rights framework. Poster presented at the American Public Health Association 141st Annual Meeting and Expo, November 2-6, 2013, Boston, MA. 